hep-th/0406001

CALT-68-2471

NSF-ITP-03-108

\title{
Superstring Orientifolds with Torsion: O5 Orientifolds of Torus Fibrations and their Massless Spectra
}

\author{
Michael B. Schulz \\ California Institute of Technology 452-48 \\ Pasadena, CA 91125 USA
}

\begin{abstract}
Using a "Superstrings with Torsion" type description, we study a class of IIB orientifolds in which spacefilling O5 planes and D5 branes wrap the $T^{2}$ fiber in a warped modification of the product of $4 \mathrm{D}$ Minkowski space and a $T^{2}$ fibration. For the case that the base is $T^{4}$, we provide examples that preserve $4 \mathrm{D} \mathcal{N}=1,2$, and 3 supersymmetry, both with internal RR flux, and with a combination of internal RR and NS flux. In these examples, the internal geometries admit integrable complex structure; however, the almost complex structure selected by the supersymmetry conditions is nonintegrable in the case that there is NS flux. We indicate explicitly the massless spectrum of gauge fields and moduli in each example. In a previous investigation, this class of orientifolds was studied using T-duality. Here, we extend the previous analysis, first by providing an intrinsic description that does not rely on duality, and then by elaborating on details of the T-duality map, which we use to check our results.
\end{abstract}

1 June 2004 


\section{Contents}

1. Introduction . . . . . . . . . . . . . . . . . . . . . . . . . . . . . 1

2. O5 Orientifolds of $T^{2}$ Fibrations . . . . . . . . . . . . . . . . . . . . . 9

3. Equations of Motion and Integrability Constraints . . . . . . . . . . . . . . . . . 14

4. Supersymmetry Conditions . . . . . . . . . . . . . . . . . . . . . . . . . . . 27

5. Relation to T-Dual O3 Orientifolds . . . . . . . . . . . . . . . . . . . . . . . 41

6. $\mathcal{N}=2$ Examples . . . . . . . . . . . . . . . . . . . . . . . . . . . . . . . . 48

7. $\mathcal{N}=3$ Example: The Warped Iwasawa Manifold . . . . . . . . . . . . . . . . . . 62

8. $\mathcal{N}=1$ Example . . . . . . . . . . . . . . . . . . . . . . . . . . . . . . . . 71

9. Preview: $\mathcal{N}=2$ Calabi-Yau Duals without Flux . . . . . . . . . . . . . . . . . . 78

10. Conclusions and Outlook . . . . . . . . . . . . . . . . . . . . . . . . . . . . . . 81

Appendices . . . . . . . . . . . . . . . . . . . . . . . . . . . . . . . . 84

\section{Introduction}

String theory admits an enormous set of seemingly consistent Minkowski vacua, yet the number of classes of compact geometries that have appeared in these vacua is small. With a few noteworthy exceptions [1,2, 3], all supersymmetric compactifications of string theory to 4D Minkowski space studied until the past two years have involved only five types of special holonomy manifolds. The canonical building blocks include Calabi-Yau threefolds, $K 3$ surfaces, and tori. Beyond these, the list also includes $G_{2}$ manifolds for $\mathrm{M}$ theory compactifications and Calabi-Yau fourfolds for F theory. It is natural to ask to what extent there exist sensible 4D Minkowski vacua based on other compactification geometries. The goal of this paper is to make contact between certain exotic orientifold vacua deduced via string dualities [4] and other work based on a "Superstrings with Torsion" type description [2].

In the absence of flux, when the geometry is a direct product of $\mathbb{R}^{3,1}$ and a compact manifold, 4D $\mathcal{N} \geq 1$ supersymmetry of the low-energy supergravity action demands that the compact manifold be a product of the special holonomy manifolds listed above [5]. In the case of type IIB string theory, a similar statement can also be made for a more general class of warped compactifications with internal flux and chiral spinor constraints. Here, the size of spacetime is allowed to vary over the internal manifold, and the data defining the compactification is enlarged to include a discrete choice of branes and internal NS and RR flux. For the class that has received the most attention, chiral (Becker-type) constraints are also imposed on the spinors generating the supersymmetries; these are the the constraints associated with D3 branes and D7 branes [6]. 
Such D3/D7 warped compactifications are attractive for a number of reasons. They provide a stringy embedding [7,8,9] of the Randall-Sundrum approach to the hierarchy problem [10], as well as a mechanism for perturbative stabilization of moduli [3, 9, 11, 12, 13, 14, 15]. (See Refs. [16, 17, 18] for a gauged supergravity perspective; also, see Ref. [19], for a discussion of local models that includes a worldsheet perspective.) In addition, they are amenable to a statistical analysis as an ensemble of vacua [20], and with a few new ingredients, have lead to a construction of metastable de Sitter vacua of string theory [21].

However, for compactification to 4D Minkowski space they require the same special holonomy manifolds as do conventional compactifications, up to an overall conformal rescaling by the warp factor [6]. Schematically, the reason is as follows. For supersymmetric IIB vacua, the gravitino variation is

$$
\delta \psi_{M}=\left(D_{M}+\text { flux }\right) \epsilon+(\text { flux }) \epsilon^{*}=0,
$$

where $\epsilon$ is a 10D Weyl spinor. In the case of Becker-type constraints, the $\epsilon$ and $\epsilon^{*}$ terms separately vanish. The $\epsilon$ term decomposes into the usual covariant constancy condition plus a relation between the RR flux $\widetilde{F}_{(5)}$ and the warp factor. The $\epsilon^{*}$ term gives conditions on the complex three-form flux $G_{(3)}$.

For other spinor constraints, or for vacua other than type IIB warped compactifications, the fluxes can mix with covariant derivatives. One obtains generalized covariant constancy conditions based on torsionful connections, whose solutions, if any, involve internal manifolds not appearing in the list above. These exotic compactification manifolds can be non-Kähler and even non-complex. However, while it is easy write down the equations of motions and supersymmetry conditions, it is not so easy to find solutions. Given certain assumptions, one can prove no-go theorems on their existence [26,9,27].2

1 An explicit model of this type with all Kähler moduli stabilized was recently exhibited in Ref. [22]. See also Ref. [23] for a nonexistence proof for one Kähler modulus, and Ref. 24] for a related discussion in the $T^{6} / \mathbb{Z}_{2}$ orientifold. For work on inflation and de Sitter space in models with D3 branes and D7 branes, see Ref. [25].

2 The no-go theorems state that in the absence of negative-tension sources, the leading $\alpha^{\prime}$ order supergravity action has no solutions with internal flux and compact internal manifold. These theorems are evaded in string theory by the existence of negative-tension orientifold planes, by $\alpha^{\prime} R \wedge R$ D7 brane worldvolume curvature couplings (which give D7 branes negative D3 brane charge and tension), and $\alpha^{\prime} R \wedge R$ corrections to the heterotic Bianchi identities. 


\subsection{Older Progress}

Most of the progress toward understanding torsionful supersymmetric vacua builds on work performed by Strominger nearly two decades ago, in the context of the heterotic string with NS flux [2]. In the heterotic theories, the gravitino and dilatino variations are

$$
\begin{aligned}
\delta \psi_{M} & =\left(\nabla_{M}+\frac{1}{4} \not H_{(3) M}\right) \epsilon, \\
\delta \lambda & =\left(\not \partial \phi+\frac{1}{2} \not H_{(3)}\right) \epsilon,
\end{aligned}
$$

where $\epsilon$ is a 10D Majorana-Weyl spinor, and $\epsilon=\left(u \otimes \chi+u^{*} \otimes \chi^{*}\right) / \sqrt{2}$ in terms of a $4 \mathrm{D}$ Weyl spinor $u$ and 6D Weyl spinor $\chi$. (Strominger did not specialize to compactifications to $4 \mathrm{D}$, but we will do so here). For supersymmetric vacua, the gravitino variation directly implies that $\chi$ and $\chi^{*}$ are both covariantly constant with respect to the same connection of torsion $T_{b c}^{a}=H_{(3)}{ }^{a} b c$. If we define an almost complex structure (ACS)

$$
J_{a}^{b}=i \chi^{\dagger} \gamma_{a}^{b} \chi
$$

then Eqs. (1.1) together imply that the ACS is integrable. Lowering one index on $J_{a}{ }^{b}$ gives the fundamental form 3 , which is related to the flux via

$$
\begin{aligned}
-e^{2 \phi} d\left(e^{-2 \phi} J\right) & =*_{6} H_{(3)}, \\
d\left(e^{-2 \phi} *_{6} J\right) & =0 .
\end{aligned}
$$

Eqs. (1.3) are not quite the relations that appeared in Strominger's work [2], but are equivalent to them [28,27].

Strominger went on to obtain a complete set of geometrical conditions for supersymmetry, as well as a set of equations expressing the dilaton and flux in terms the geometry. In addition, he provided a worldsheet description, and for compactifications to 6D was able to give exact solutions. For compactifications to $4 \mathrm{D}$ on a compact manifold other than a Calabi-Yau, he argued that the solutions could not extend to large volume, since his equations reduced to Calabi-Yau conditions in this limit. He did not provide any solutions, but did mention the Iwasawa manifold as an example of a complex non-Kähler manifold satisfying the topological condition $h^{3,0}=1$, that could conceivably be used as the basis for a consistent torsionful supersymmetric solution.

3 In the special case that $J$ is closed, the internal manifold is Kähler and $J$ is the Kähler form.

4 Heterotic compactifications on the Iwasawa manifold have been re-explored using the tools discussed in the first part of Sec. 1.2 [29]. In Sec. 7, we discuss orientifolds based on a warped Iwasawa manifold. 
This is the way things stood for thirteen years until the first non-Kähler heterotic compactifications to 4D were constructed using string dualities [3]. The starting point was a IIB warped compactification, obtained as the orientifold limit of a warped F-theory compactification on $K 3 \times K 3$ with internal flux [30,31]. After two T-dualities, 5 this theory becomes type I on a non-Kähler manifold with RR three-form flux. The heterotic theory is then obtained via S-duality. The resulting geometry takes the form of a warped $T^{2}$ fibration over $K 3$, with the noteworthy property that the fiber volume is fixed in terms of the fiber complex structure modulus. So, lengths cannot simply be scaled by an overall factor to obtain a large volume solution, in agreement with Strominger's earlier observation forbidding an overall volume modulus.

\subsection{More Recent Progress}

Two important organizing principles that have emerged more recently are the notions of $G$-structures and generalized calibrations. These tools were first applied to studying supergravity solutions with background flux in Ref. [28]. Since then, the subject has flourished $[32,29,33,27,34,35,36,37,38,39,40,41,42,43,44$. In the context of the heterotic string with $4 \mathrm{D} \mathcal{N}=1$ supersymmetry, the appropriate group $G$ is $S U(3)$, and the idea is as follows [32,29,27]. The existence of the covariantly constant spinor $\chi$ discussed above is equivalent to the statement that the compactification manifold $X_{6}$ has $S U(3)$ holonomy with respect to the torsionful connection. As a consequence of the existence of a privileged $6 \mathrm{D}$ spinor, the usual $S U(3)$ structures $J$ and $\Omega$ can be canonically defined in terms of this spinor, just as for a Calabi-Yau. However, these objects are no longer closed. A useful mathematical characterization of the precise sense in which $X_{6}$ differs from a Calabi-Yau comes from the fact that the $S U(3)$ structures induce a natural $S U(3)$ decomposition of the torsion into five torsion classes [45]:

$$
T=\underset{(1+1)}{\mathcal{W}_{1}}+\underset{(8+8)}{\mathcal{W}_{2}}+\underset{(6+\overline{6})}{\mathcal{W}_{3}}+\underset{(3+\overline{3})}{\mathcal{W}_{4}}+\underset{(3+\overline{3})}{\mathcal{W}_{5}}
$$

In terms of this decomposition,

$$
\begin{aligned}
& d J=\frac{3}{4} i\left(\mathcal{W}_{1} \bar{\Omega}-\overline{\mathcal{W}}_{1} \Omega\right)+\mathcal{W}_{3}+J \wedge \mathcal{W}_{4}, \\
& d \Omega=\mathcal{W}_{1} J \wedge J+J \wedge \mathcal{W}_{2}+\Omega \wedge \mathcal{W}_{5} .
\end{aligned}
$$

5 The geometry of the IIB orientifold is a warped version of $\mathbb{R}^{3,1} \times K 3 \times T^{2} /\left(\Omega(-1)^{F_{L}} \mathcal{I}_{2}\right)$, where $\mathcal{I}_{2}$ inverts the $T^{2}$. The $\mathrm{T}$-dualities are performed in the $T^{2}$ directions. 
The supersymmetry conditions can then be translated into constraints on the $\mathcal{W}_{i}$. Conversely, once these constraints are known, we can instead start with a manifold satisfying the constraints, and then $H_{(3)}$ and $e^{\phi}$ follow from Eqs. (1.3).

The connection to generalized calibrations arises when, following Ref. [28,27], we interpret Eq. ( $1.3 d)$ as a consequence of the fact that (i) NS5 branes are a source of $H_{(3)}$ flux, and (ii) $J$ is a generalized calibration [46] for the two-cycles on which we can wrap a supersymmetric NS5 brane probe. In some sense, we can think of the manifold $X_{6}$ as a fully backreacted geometry involving one or more wrapped NS5 branes. The appeal of this formalism is that it readily generalizes. Instead of considering the heterotic string on a 6D manifold with a torsionful connection of $S U(3)$ holonomy, we can consider type I, II, or the heterotic theories on a manifold of some other dimension, with torsionful connection of holonomy group $G$. The $S U(3)$ structures $J$ and $\Omega$ just get replaced by the appropriate $G$-structures. The generalization of Eq. (1.3a) is an analogous relation between some NS or RR flux and the $G$-structure that calibrates the cycles on which we can wrap a corresponding NS or D brane probe.

Beyond $G$-structures, another important advance has been the reformulation of the heterotic moduli constraints in a way analogous to the usual formulation of the constraints for IIB Becker-type warped compactifications. For the latter, one defines a complex flux $G_{(3)}=F_{(3)}-\tau_{\text {dil }} H_{(3)}$. The supersymmetry conditions constrain the moduli so that $G_{(3)}$ is primitive 6 and of type $(2,1)$. The $(2,1)$ condition follows from a superpotential $W=$ $\int G_{(3)} \wedge \Omega$. Finally, the scalar potential comes from Kaluza-Klein reduction of the kinetic term for $G_{(3)}$ in the $10 \mathrm{D}$ supergravity action. A similar structure has been exhibited in the heterotic theories, except that the scalar potential now comes from a combination of the $H_{(3)}$, Einstein-Hilbert, and dilaton kinetic terms in the 10D supergravity action [47]. The complex flux $H_{(3)}-i e^{2 \phi} d\left(e^{-2 \phi} J\right)$ is required to be $(2,1)$ and primitive. It has been argued that the $(2,1)$ condition follows from an analogous superpotential 448,47,49, although this is more subtle than in the IIB case.

The duality chains, which proved so useful in the first non-Kähler heterotic compactifications to four dimensions, have also been revisited. In the original construction [3], all analysis was performed in the dual IIB/F-theory description. Much effort has been devoted to providing an intrinsically heterotic description of these vacua and their generalizations, as well as to the mathematical properties of the compact manifolds involved [48,50,49].

\footnotetext{
${ }^{6}$ Primitivity means that $J \wedge G_{(3)}=0$.
} 
From the results of Ref. [49]: the previously known class of admissible compact manifolds obtained via duality has been generalized to include manifolds with nonzero Euler characteristic, and a better understanding now exists of phenomenologically relevant details concerning the vector bundles on these manifolds and the numbers of fermion generations; moreover, through a different duality, there is now a relation to brane-box constructions. A particular duality symmetry that has proven extremely useful in the past is mirror symmetry, and steps have been taken toward generalizing Calabi-Yau mirror symmetry to a mirror symmetry of non-Kähler manifolds with nonvanishing NS flux [4, 32, 38]. Even for geometric transitions, the cornerstones in our modern understanding of topological string theory [51], there now exists a sequence of supergravity descriptions for a complete duality cycle connecting the various IIB, IIA, and M theory descriptions before and after the transition [52]. This duality cycle relates D branes wrapped on cycles of non-Kähler manifolds to fluxes in other non-Kähler manifolds. For other interesting results involving dualities and torsion, see Ref. [53].

\subsection{Work Reported Here}

A final way in which duality symmetries have been applied is in the construction of a new class of non-Kähler orientifold vacua 四. These vacua will be our focus here. They are relatively simple to describe - the geometry is a warped torus fibration over a torus base, with $\mathrm{O}$ planes and D branes wrapping the fiber and filling spacetime. In addition, there is internal flux. These vacua were constructed using a duality argument similar to the one used to obtain non-Kähler heterotic compactifications. However, the orientifold vacua that we discuss here do not have obvious geometrical heterotic duals. The starting point is a $T^{6}$ orientifold of type IIB with internal flux, where the orientifold inverts all of the torus directions. So, the initial theory contains O3 planes and possibly D3 branes, but no O7 planes or D7 branes. The non-Kähler orientifolds are obtained via T-duality. For supersymmetric flux, the maximum number of T-dualities that can be performed is either two or three, 1 depending on the choice of flux. Therefore, we cannot relate these string vacua to type I with O9 planes, or subsequently to the heterotic theory by S-duality.

7 After this number of T-dualities, the metric has no further isometries that can be used to perform additional standard T-dualities. There does exist a generalized sense in which we can perform additional T-dualities. However, the result is a nongeometrical string compactification 54,4, involving duality twists that mix the metric and NS $B$-field, so that neither of these quantities is globally well-defined by itself. In contrast, standard T-dualities only result in geometric fibrations (Scherk-Schwarz [55] twists of the metric) and NS flux (Scherk-Schwarz twists of the NS $B$-field). 
The main motivation for the present investigation is that the orientifolds just described have been analyzed in a way that relies almost exclusively on the original pre-duality theory, with little or no intrinsic description in the final non-Kähler orientifold. On the other hand, the geometry of these orientifolds is very similar to that of certain noncompact manifolds of $S U(2)$ or $S U(3)$ structure that have been described elsewhere, and used in consistent supergravity solutions with a "Superstrings with Torsion" type description [27]. The primary goal below is to provide such a description for torsionful orientifolds of type IIB string theory in which O5 planes are wrapped on the fiber of a $T^{2}$ fibration. A secondary goal is to study moduli stabilization in these orientifolds.

An outline of the paper is as follows. We begin in Sec. 2 by defining the class of O5 orientifolds that we consider. Sec. 2.1 contains a description of the 10D supergravity background, including the $10 \mathrm{D}$ metric, the orientifold operation, and fluxes. In Secs. 2.2 and 2.3 we discuss the fields and energy scales of the $4 \mathrm{D}$ effective field theory.

Sec. 3 is devoted to analyzing the model to the extent possible without any assumptions regarding supersymmetry. Here, and also in Sec. 4, we relax the ansatz that the base of the internal manifold is $T^{4}$, and assume only that it is some compact manifold $\mathcal{B}$. However, order to proceed systematically, we find it necessary to impose one restriction on the NS flux. This restriction is discussed at the end of Sec. 3.1. The main results of Sec. 3 are the Gauss's law constraint in Sec. 3.2, a pseudo-BPS constraint on the fluxes in Sec. 3.3, expressions for the dilaton and warp factor in Secs. 3.4 and 3.5, and the requirement that the base be Ricci flat. The pseudo-BPS constraint follows from an equality between an exact four-form on the base and sum of positive semidefinite squares of fluxes. Integration of this relation imposes a set of Hodge duality conditions on the fluxes. There is a naive paradox concerning the Gauss's law constraint. Due to the fibration, one can have $d \widetilde{F}_{(3)}$ supported on local sources, the sum of whose charges does not vanish. This paradox is resolved by correctly pushing forward the $\widetilde{F}_{(3)}$ Bianchi indentity to the base. We conclude the section with a precise definition of the moduli, and a decomposition of the fluxes into a quantized discrete part and moduli dependent deformations.

Sec. 4 contains a discussion of the supersymmetry conditions. After a brief discussion in Sec. 4.1 of the contraints on the spinors from the orientifold projection, in Sec. 4.2 we analyze the case in which only the RR three-form flux is nonzero. Here, the discussion closely parallels that given by Strominger for the heterotic string with NS flux. The vacua that we describe are related by S-duality to dual vacua with NS-flux, NS5 branes, and ON5 planes, that are very similar to those discussed by Gauntlett, Martelli, and Waldram 
[27] in the case of $6 \mathrm{D}$ internal manifold. The S-duality map is discussed in Sec. 4.3. We then consider the case of more general flux in Sec. 4.4, and derive the corresponding generalization of the supersymmetry conditions. These conditions are stated in terms of the flux, the right- $S U(3)$ structures $J$ and $\Omega$ constructed from the right-moving part of the Killing spinors only (or alternatively, left- $S U(3)$ structures), and the volume form $V_{\text {ol }}$ fib on the $T^{2}$ fiber. Note that while we employ $S U(3)$ structures in analyzing the supersymmetry conditions, we do not find it useful here to work in terms of $S U(3)$ (or $S U(2)$ ) torsion classes in order to satisfy these conditions. Therefore, we will not compute torsion classes in this paper, except for a fleeting instance in Sec. 6.3 to verify the nonintegrability of the almost complex structure. See, however, the discussion in Sec. 10.

In Sec. 5, we relate O5 orientifolds with $T^{4}$ base to T-dual O3 orientifolds with internal $T^{6}$. Then, in Secs. 6-8 we study a number of specific classes of O5 backgrounds preserving various amounts of supersymmetry, and their O3 duals. Sec 6.3 is devoted to a class with nonvanishing $F_{(1)}$ and $H_{(3)}$ flux, and nonintegrable complex structure, but the examples in Secs. 6-8 otherwise contain only $\widetilde{F}_{(3)}$ flux and integrable complex structure. Our discussion of the $\mathrm{O} 3$ duals extends the known results for the $T^{6} / \mathbb{Z}_{2}$ orientifold.

Sec. 9 contains a preview of work to appear elsewhere [56. In the $\mathcal{N}=2$ case, our examples are dual to $\mathrm{M}$ theory on a Calabi-Yau threefold $Y_{6}$ times a circle. That is, they can be viewed as $\mathrm{F}$ theory on $Y_{6} \times T^{2}$, where the $\mathrm{F}$ theory fiber is taken to be the product of an $S^{1}$ in the $T^{2}$ and an $S^{1}$ in $Y_{6}$ (with $Y_{6}$ viewed as a fibration). Here, we provide a few preliminary observations concerning this duality.

Finally, in Sec. 10, we conclude and discuss possibilities for future work.

The appendices contain auxilliary details not found in the body of the paper. App. A contains a summary of our conventions, mostly with regard to Dirac matrices. In App. B, we describe the twisted coordinate identifications that generalize the $T^{6}$ identifications $x^{a} \cong x^{a}+1$ to a $T^{2}$ fibration over $T^{4}$. In App. C, we derive the first equation of Sec. 3.3, from which the pseudo-BPS constraints follow. App. D is a reference for the IIB supersymmetry variations of fermions in various forms (string frame, Einstein frame, in terms of $\epsilon_{L, R}$ and in terms of $\left.\epsilon=\epsilon_{L}+i \epsilon_{R}\right)$. In App. E, we discuss subtleties in applying the superpotential of Gukov, Vafa, and Witten [57] to the $T^{6} / \mathbb{Z}_{2}$ O3 orientifold in which the fluxes (partially) break $\mathcal{N}=4$ extended supersymmetry. The T-duality map of RR moduli, as defined in Secs. 3.9 and 5.1, is worked out in App. F. App. G contains a discussion of moduli space metrics for the $T^{6} / \mathbb{Z}_{2}$ orientifold; here, we first review the results of Frey and Polchinski for the case of $\mathcal{N}=3$ supersymmetry, and then consider the $\mathcal{N}=2,1$ 
cases relevant to Secs. 6 and 8. Lastly, in App. H we prove that a particular class of fluxes discussed in Sec. 8.2 reduces to a unique choice of flux modulo the discrete identifications of the axion-dilaton and $T^{6}$ complex structure.

\section{O5 Orientifolds of $T^{2}$ Fibrations}

\subsection{Supergravity Background}

As a starting point, recall that the supergravity background for $N$ coincident D5 branes in flat noncompact spacetime is 58

$$
\begin{aligned}
d s_{\text {string }}^{2} & =Z^{-1 / 2} \eta_{\mu \nu} d x^{\mu} d x^{\nu}+Z^{1 / 2} \delta_{m n} d x^{m} d x^{n} \\
e^{\phi} & =g_{s} Z^{-1 / 2} \\
F_{(7)} & =-g_{s}^{-1} d\left(Z^{-1}\right) \wedge d x^{0} \wedge \ldots \wedge d x^{5}
\end{aligned}
$$

where $\mu, \nu=0, \ldots, 5$ and $m, n=6, \ldots, 9$. The subscript "string" indicates that Eq. (2.10) gives the string frame metric. The warp factor is

$$
Z=1+N \frac{\alpha^{\prime} g_{s}}{2 r^{2}}
$$

This function is harmonic away from $r=0$ and is a solution to Poisson's equation

$$
\delta^{m n} \partial_{m} \partial_{n} Z=N(2 \pi)^{2} \alpha^{\prime} g_{s} \delta^{4}(x)
$$

in the non-warped metric $\delta_{m n}$ on the $\mathbb{R}^{4}$ transverse to the D5 branes.

We would like to study the analogous supergravity backgrounds for orientifolds of type IIB string theory in which 4D spacetime-filling O5 planes and D5 branes wrap the $T^{2}$ fiber over a $T^{4}$ base. 8 The new metric ansatz is

$$
d s_{\text {string }}^{2}=Z^{-1 / 2}\left(\eta_{\mu \nu} d x^{\mu} d x^{\nu}+d s_{T_{\text {fib }}^{2}}^{2}\right)+Z^{1 / 2} d s_{T_{\text {base }}^{4}}^{2}
$$

where $\eta_{\mu \nu}$ is the 4D Minkowski metric, and the fiber and base metrics are

$$
\begin{aligned}
d s_{T_{\text {fib }}^{2}}^{2} & =g_{\alpha \beta}^{\left(T_{\text {fib }}^{2}\right)} \eta^{\alpha} \eta^{\beta}, \quad \eta^{\alpha}=d x^{\alpha}+\mathcal{A}^{\alpha}, \quad \alpha, \beta=4,5, \\
d s_{T_{\text {base }}^{4}}^{2} & =g_{m n}^{\left(T_{\text {base }}^{4}\right)} d x^{m} d x^{n}, \quad m, n=5, \ldots, 9 .
\end{aligned}
$$

8 Although we focus on the case of $T^{4}$ base, most of the analysis in Secs. 3 and 4 is performed for arbitrary compact base $\mathcal{B}$. 
Here, we assume that $g_{\alpha \beta}^{\left(T_{\text {fib }}^{2}\right)}$ and $g_{m n}^{\left(T_{\text {base }}^{4}\right)}$ are flat metrics, and that the warp factor depends only on the base coordinates. The fiber and base coordinates are identified via a twisted generalization of $x^{\alpha} \cong x^{\alpha}+1, x^{m} \cong x^{m}+1$ that is discussed in App. B. The quantity $\mathcal{A}^{\alpha}=\mathcal{A}^{\alpha}{ }_{m}\left(\left\{x^{n}\right\}\right) d x^{m}$ is the fiber connection. The one-forms $\eta^{\alpha}$ are globally defined and are the generalizations of $d x^{\alpha}$ consistent with the twisting of the fibration. Nontrivial twisting is measured by nonvanishing cohomology classes

$$
\left[\mathcal{F}^{\alpha}\right] \in H^{2}\left(T_{\text {base }}^{4}, 2 \mathbb{Z}\right)
$$

where

$$
\mathcal{F}^{\alpha}=d \mathcal{A}^{\alpha}
$$

These are the Chern classes of the $x^{\alpha}$ circle fibrations (or equivalently $U(1)$ fibrations) over $T_{\text {base }}^{4}$.

For later convenience, we define a $6 \mathrm{D}$ metric

$$
d s_{6}^{2}=d s_{T_{\mathrm{fib}}^{2}}^{2}+Z d s_{T_{\mathrm{base}}^{4}}^{2}
$$

so that

$$
d s_{\text {string }}^{2}=Z^{-1 / 2}\left(\eta_{\mu \nu} d x^{\mu} d x^{\nu}+d s_{6}^{2}\right)
$$

We denote by $X_{6}$ the $6 \mathrm{D}$ space whose metric is $(2.7)$.

In addition to this geometry, the model includes $16 \mathrm{O} 5$ planes and $2 M$ D 5 branes. The $\mathbb{Z}_{2}$ orientifold operation that defines the $\mathrm{O} 5$ planes is $\Omega \mathcal{I}_{4}$, where $\Omega$ is worldsheet parity and $\mathcal{I}_{4}$ inverts the base $T^{4}$ :

$$
\mathcal{I}_{4}: \quad x^{m} \rightarrow-x^{m}
$$

The orientifold planes wrap the fiber and are located at the $2^{4} \mathbb{Z}_{2}$ fixed points on the base where $x^{m}=0,1 / 2.9$ The $2 M$ D5 branes also wrap the fiber, and are located at $M$ arbitrary points on the base together with their $M \mathbb{Z}_{2}$ images. The $2 \mathbb{Z}$ rather than $\mathbb{Z}$ valued cohomology in Eq. (2.5) ensures that $\left[\mathcal{F}^{\alpha}\right] \in H^{2}\left(T^{4} / \mathcal{I}_{4}, \mathbb{Z}\right)$, which is needed to define the orientifold. (See Sec. 3.7).

9 The orientifold planes are assumed to be standard $05^{-}$planes in the terminology of [59, as opposed to the exotic $\mathrm{O}^{+}$or $\widetilde{\mathrm{O}} 5^{ \pm}$planes that lead to shifted flux quantization conditions. Similarly, we assume that there is no localized $\mathcal{F}^{\alpha}$ curvature at the $\mathcal{I}_{4}$ fixed points. 
Finally, the orientifold projection preserves the following even fluxes together with their 10D Hodge duals 10

$$
\text { Even fluxes: } \quad \widetilde{F}_{(1) m}, \quad \widetilde{F}_{(3) \alpha m n}, \quad \widetilde{F}_{(5) \alpha \beta m n p}, \quad H_{(3) \alpha \beta m}, \quad H_{(3) m n p} .
$$

By even, we mean that these components must be even functions of the transverse coordinates $x^{m}$. The orientifold projection also preserves the complementary set of components, provided these components are odd functions of the transverse coordinates. With the exception of the special case that $2 M=32$ and each $\mathrm{O} 5$ plane is coincident with exactly one D5 brane and its image (so that charges and tensions cancel locally), there do not exist string vacua with all odd fluxes set to zero. In order to satisfy the low energy equations of motion, we need to include at least the following odd flux:

$$
\text { Odd flux: } \widetilde{F}_{(3) m n p} \text {. }
$$

We will limit our investigation to those backgrounds in which the other odd fluxes can be consistently set to zero, and in which all fields have functional dependence on the base coordinates only. (In other words, we consider the low energy effective field theory at energy scales below $1 / R_{\text {fib }}$; see Sec. 2.3 for further discussion.) In addition, we assume vanishing field strengths of the D5 worldvolume gauge fields.

The fluxes $\widetilde{F}_{(p)}$ are the gauge-invariant fluxes that appear in the kinetic terms of the bulk supergravity action. In the conventions in which the T-duality action on RR potentials is simplest, 1 the relation between the RR potentials $C_{(p)}$ and gauge-invariant fluxes $\widetilde{F}_{(p)}$ is

$$
\widetilde{F}_{(p)}= \begin{cases}F_{(p)}-C_{(p-3)} \wedge H_{(3)} & p \geq 3 \\ F_{(p)} & p=1,2\end{cases}
$$

where

$$
F_{(p)}=d C_{(p-1)} .
$$

In Sec. 3, we will see that the odd flux (2.11) is completely determined by the equations of motion. On the other hand, the even fluxes (2.10) contain both moduli-dependent and moduli-independent components, with the latter constrained only by Dirac quantization.

10 Here, all components of fluxes and potentials are given in the basis $d x^{\mu}, \eta^{\alpha}, d x^{m}$. These components should not be confused with those in the basis $d x^{m}, d x^{\alpha}, d x^{m}$.

11 There is different convention, $\widetilde{F}_{(5)}=F_{(5)}+\frac{1}{2} B_{(2)} \wedge F_{(3)}-\frac{1}{2} C_{(2)} \wedge H_{(3)}$, in which the potentials $B_{(2)}$ and $C_{(2)}$ transform simply under $S L(2, \mathbb{Z})$ duality. 
Therefore, the even fluxes, like $\mathcal{F}^{\alpha}$ and $M$, contain discrete data that needs to be specified in order to fully define the model.

For trivial fibration and in the absence of even flux, this orientifold is dual to type I on $T^{6}$ via $T$-duality in the four $T_{\text {base }}^{4}$ directions. In this case, the orientifold preserves $4 \mathrm{D}$ $\mathcal{N}=4$ supersymmetry. Also, since the D5 branes and O5 planes fill 4D spacetime, there is a Gauss's law constraint ( $\mathrm{RR}$ tadpole cancellation condition in worldsheet language) that requires $2 M=32 \mathrm{D} 5$ branes. This constraint and its generalization to the case of nontrivial fibration and flux are discussed in Sec. 3.2 below.

\subsection{Low Energy Bosonic Field Content}

For the class of 10D supergravity backgrounds just described, there exist deformations that continuously connect different consistent supergravity solutions. Let us restrict to deformations with no functional dependence on $x^{\mu}$. For the case of trivial fibration and in the absence of even flux, one class of such deformations consists of the zero modes on $X_{6}$ of the even bosonic fields

$$
\begin{aligned}
\text { RR sector: } & C_{(2) \alpha \beta}, \quad C_{(2) \mu \nu} \leftrightarrow \text { scalar, } C_{(2) m n}, C_{(4) \alpha m n p}, \\
\text { NSNS sector: } & g_{\alpha \beta}, \quad g_{m n}, \quad B_{(2) \alpha m}, \quad \phi
\end{aligned}
$$

together with the zero modes on the $T^{2}$ fiber of the D5 worldvolume fields

$$
\text { D5 worldvolume: } \quad A_{I \alpha}, \quad \Phi_{I}^{m}, \quad I=1, \ldots, M \text {. }
$$

These are the deformations which, when promoted to $4 \mathrm{D}$ fields, become the moduli of the $4 \mathrm{D} \mathcal{N}=4$ low energy effective field theory.

The quantity that plays the role of the axion-dilaton is (cf. Sec. 4.2)

$$
\tau_{\text {dil }}=\frac{1}{(2 \pi)^{2} \alpha^{\prime}}\left(-C_{(2)} 45+\frac{i}{g_{s}} V_{\text {fib }}\right)
$$

where the volume of the $T^{2}$ fiber is

$$
V_{\mathrm{fib}}=\left(g^{\left(T_{\mathrm{fib}}^{2}\right)}\right)^{1 / 2}
$$

This expression for $\tau_{\text {dil }}$ is T-dual to the more familiar expression given App. D that is common to $\mathrm{O} 3$ orientifolds, O7 orientifolds, and pure IIB string theory. 
A second class of such deformations consists of the zero modes on $X_{6}$ of the even fields

$$
\begin{array}{rll}
\text { RR sector: } & C_{(2) \alpha \mu}, \quad C_{(4) \alpha \beta m \mu}, \\
\text { NSNS sector: } & V^{\alpha}{ }_{\mu}, \quad B_{(2) m \mu}, \\
\text { D5 worldvolume: } & A_{I \mu}, \quad I=1, \ldots, M .
\end{array}
$$

These are the deformations which, when promoted to $4 \mathrm{D}$ fields, become the massless gauge bosons of the $4 \mathrm{D} \mathcal{N}=4$ low energy effective field theory. Here, $V^{\alpha}{ }_{\mu}$ is the deformation that corresponds to the Kaluza-Klein gauge boson for the translation isometry in the $\alpha$-direction. It arises by replacing $\mathcal{A}^{\alpha}$ with $\mathcal{A}^{\alpha}+V^{\alpha}{ }_{\mu} d x^{\mu}$ in the 10D metric (2.4b).

Finally, a third class of such deformations shifts the $4 \mathrm{D}$ metric away from $\eta_{\mu \nu}$ and parametrizes the space of constant metrics on $\mathbb{R}^{3,1}$. When promoted to $4 \mathrm{D}$ fields, these deformations become the 4D graviton.

Note that there is an important distinction between the allowed $x^{\mu}$-independent deformations of the supergravity backround and the fields of the 4D effective action. While it is easy to identify the former, the massless fields in the dimensional reduction to $4 \mathrm{D}$ are more complicated than those obtained by simply endowing these deformations with $x^{\mu_{-}}$ dependence. The correct low energy $4 \mathrm{D}$ fields must also include warp factor dependence to prevent mixing with higher Kaluza-Klein modes [60, 15, 61].

In the case of nontrivial fibration and flux, the supersymmetry and massless field content is reduced. The $U(1)^{M} \mathcal{N}=4$ vector multiplets on the D5 branes remain massless, but many of the RR and NS fields in (2.14) and (2.17) are lifted. The lifting occurs through a supersymmetric Higgs mechanism. For the bosons, the equations of motion impose certain metric and axion-dilaton dependent Hodge duality relations on the fluxes that lift a subset of the NS moduli and the zero mode of the RR axion $C_{(2)} 45$. The vevs of some of these moduli then break a subset of the gauge symmetries, and their axionic partners (zero modes of some of the $C_{(2) m n}$ and $C_{(2) \alpha m n p}$ ) are eaten by massive vectors. The fermions also take part in the superHiggs mechanism, and the various possibilities for the resulting $\mathcal{N}<4$ low energy spectrum are determined by the masses of the $(4-\mathcal{N})$ massive gravitini, as described in Ref. [17]. (See also Ref. [16]).

\subsection{Energy Scales}

There are two points of view that we can adopt regarding the moduli stabilization and symmetry breaking just described. If we assume that the fiber and base have roughly the 
same length scale $R$, then these effects occur at the Kaluza-Klein scale $1 / R$. However, if we assume a hierarchy $R_{\mathrm{fib}} \ll R_{\text {base }}$, then these effects occur at the scale $m=R_{\mathrm{fib}} / R_{\text {base }}^{2}$ [32], which is much smaller than $m_{\mathrm{base}}^{\mathrm{KK}}=1 / R_{\text {base }}$ and $m_{\mathrm{fib}}^{\mathrm{KK}}=1 / R_{\mathrm{fib}}$ :

$$
m: m_{\mathrm{base}}^{\mathrm{KK}}: m_{\mathrm{fib}}^{\mathrm{KK}}=1: R_{\mathrm{base}} / R_{\mathrm{fib}}:\left(R_{\mathrm{base}} / R_{\mathrm{fib}}\right)^{2} .
$$

In the former case, it only makes sense to talk about a low energy $\mathcal{N}<4$ theory with all massive fields integrated out. In the latter, there is a consistent low energy $4 \mathrm{D}$ supergravity theory within which $\mathcal{N}=4$ is softly broken to $\mathcal{N}<4$ by a supersymmetric Higgs mechanism. We will adopt the latter point of view below. In either case, we need $R_{\text {base }}, R_{\mathrm{fib}} \gg \alpha^{\prime}$ in order to be able to neglect higher string modes.

\section{Equations of Motion and Integrability Constraints}

Before imposing the supersymmetry conditions, let us first determine the constraints that follow from equations of motion alone.

\subsection{Equations of Motion}

The trace-reversed Einstein equations are

$$
R_{M N}=\widetilde{T}_{M N}
$$

where $\widetilde{T}_{M N}=T_{M N}-\frac{1}{8} g_{M N} T$.

The components of the Ricci tensor in the metric (2.4) are

$$
\begin{aligned}
R_{\mu \nu}= & \nabla_{\mu} \nabla_{\nu} \log Z+\frac{1}{4} Z^{-1 / 2} \nabla_{\mathcal{B}}^{2} \log Z \eta_{\mu \nu}, \\
R_{\alpha \beta}= & \nabla_{\alpha} \nabla_{\beta} \log Z+\frac{1}{4} Z^{-1 / 2} \nabla_{\mathcal{B}}^{2} \log Z \delta_{\alpha \beta}+\frac{1}{4} \mathcal{F}_{\alpha \beta}^{2}, \\
R_{m n}= & \nabla_{m} \nabla_{n} \log Z-\frac{1}{4} Z^{-1 / 2} \nabla_{\mathcal{B}}^{2} \log Z \delta_{m n}-\frac{1}{2} \mathcal{F}_{m n}^{2} \\
& -\frac{1}{2}\left(\nabla_{\mathcal{B} m} \log Z\right)\left(\nabla_{\mathcal{B} n} \log Z\right)+R_{\mathcal{B} m n} .
\end{aligned}
$$

Here $\mathcal{B}$ denotes the $4 \mathrm{D}$ base of the torus fibration. Most of the results of Secs. 3 and 4 are independent of the choice $\mathcal{B}=T^{4}$, so we work with arbitrary $\mathcal{B}$. The operator $\nabla_{\mathcal{B}}$ is the Levi-Civita covariant derivative on the base, and the Laplacian $\nabla_{\mathcal{B}}^{2}$ is contracted using the base metric

$$
d s_{\mathcal{B}}^{2}=g_{\mathcal{B} m n} d x^{m} d x^{n}
$$


that generalizes Eq. (2.4d).

In string frame, the trace-reversed stress tensor is $\hat{T}_{M N}=\widetilde{T}_{M N}^{\mathrm{NS}}+\widetilde{T}_{M N}^{\mathrm{RR}}+\widetilde{T}_{M N}^{\text {local }}$, where

$$
\begin{aligned}
& \widetilde{T}_{M N}^{\mathrm{NS}}=-2 \nabla_{M} \nabla_{N} \phi+\frac{1}{2} \frac{1}{2 !} H_{(3) M N}^{2}, \\
& \widetilde{T}_{M N}^{\mathrm{RR}}=\frac{1}{2} e^{2 \phi} \sum_{p=1,3}\left(\frac{1}{(p-1) !} \widetilde{F}_{(p) M N}^{2}-\frac{1}{2} g_{M N} \frac{1}{p !} \widetilde{F}_{(p)}^{2}\right)+\frac{1}{4} e^{2 \phi}\left(\frac{1}{4 !} \widetilde{F}_{(5) M N}^{2}-\frac{1}{2} g_{M N} \frac{1}{5 !} \widetilde{F}_{(5)}^{2}\right), \\
& \widetilde{T}_{M N}^{\text {local }}=-(2 \pi)^{2} \alpha^{\prime} \frac{1}{2} e^{\phi} \sum_{i}\left(h_{M N}\left(x_{i}\right)-\frac{1}{2} g_{M N}\right) Q_{i} \frac{\delta^{4}\left(x-x_{i}\right)}{Z \sqrt{g_{\mathcal{B}}}} .
\end{aligned}
$$

Here, $h_{M N}\left(x_{i}\right)$ is the restriction of the 10D metric (2.4) to the point $x=x_{i}$ in the base,

$$
d s_{h\left(x_{i}\right)}^{2}=\left.Z^{-1 / 2}\left(\eta_{\mu \nu} d x^{\mu} d x^{\nu}+g_{\alpha \beta}^{\left(T_{\mathrm{fib}}^{2}\right)} \eta^{\alpha} \eta^{\beta}\right)\right|_{x^{m}=x_{i}^{m}}
$$

The sum on $i$ runs over D5 brane and O5 plane sources of charge $Q_{i}$ in units of D5 brane charge, localized at points $x=x_{i}$ on the base. For $\mathcal{B}=T^{4}$, this includes $2 M$ D5 branes of charge $Q_{i}=+1$ and 16 O5 planes of charge $Q_{i}=-2$ located at the $\mathbb{Z}_{2}$ fixed points. We work on the covering space of the orientifold, so that there are $M$ independent D5 branes at $x_{I}, I=1, \ldots, M$, and $M$ image D5 branes located at $-x_{I}$. The 05 plane charge on the covering space is twice the O5 charge of the "downstairs" picture.

The dilaton equation of motion is

$$
\nabla^{2} \phi-2(\nabla \phi)^{2}=e^{2 \phi}\left(F_{(1)}^{2}+\frac{1}{2} \frac{1}{3 !} \widetilde{F}_{(3)}^{2}\right)-\frac{1}{2} \frac{1}{3 !} H_{(3)}^{2}+(2 \pi)^{2} \alpha^{\prime} \frac{1}{2} e^{\phi} \sum_{i} Q_{i} \frac{\delta^{4}\left(x-x_{i}\right)}{Z \sqrt{g_{\mathcal{B}}}}
$$

Finally, the Bianchi identity for $\widetilde{F}_{(3)}$ is

$$
d \widetilde{F}_{(3)}=-F_{(1)} \wedge H_{(3)}-(2 \pi)^{2} \alpha^{\prime} \operatorname{Vol}_{\mathcal{B}} \sum_{i} Q_{i} \frac{\delta^{4}\left(x-x_{i}\right)}{\sqrt{g_{\mathcal{B}}}}
$$

where $\mathrm{Vol}_{\mathcal{B}}$ is the volume form on the base,

$$
\mathrm{Vol}_{\mathcal{B}}=\left(g_{\mathcal{B}}\right)^{1 / 2} d x^{6} \wedge d x^{7} \wedge d x^{8} \wedge d x^{9}
$$

There are additional equations of motion and Bianchi identities for the fluxes. These are discussed in Sec. 3.8 below.

In order to make the equations of motion tractable, we will now impose one further restriction on supergravity backgrounds that we consider. We demand that $H_{(3) \alpha \beta m}=0$. 
This condition is equivalent to restricting to the subset of vacua of this orientifold that are related to D3/O3 orientifold vacua via T-duality in the fiber directions.12 We have not succeeded in constructing supergravity solutions with $H_{(3) \alpha \beta m} \neq 0$. One of the complications associated with this case is that the pseudo-BPS constraints of Sec. 3.3 apparantly no longer hold. The flux $H_{(3) \alpha \beta m}$ contributes negatively to an otherwise positive semidefinite sum of squares. (See App. C for further discussion of this point). More significant qualitative differences implied by $H_{(3) \alpha \beta m}$ nonzero are (i) $B_{(2) \alpha \beta}$ is nonzero, so that there is noncommutativity on the D5 branes, and/or (ii) there does not exist a consistent truncation of the supergravity action to the lowest Kaluza-Klein modes. See Ref. [62] for a discussion of the gauge algebra for certain supergravity backgrounds with $H_{(3) \alpha \beta m} \neq 0$ and positive $4 \mathrm{D}$ vacuum energy.

\subsection{Gauss's Law Constraint}

Since the O5 planes and D5 branes fill all of the noncompact Minkowski directions, we expect the Bianchi identity (3.7) to imply an integrability condition that roughly demands that the total D5 charge vanish.

In order to derive this constraint, let us first introduce a notation that will be useful throughout the paper. For any $p$-form $\omega_{(p)}$ on $X_{6}$, we write

$$
\omega_{(p)}=\omega_{(p)}^{0}+\omega_{(p)}^{1}+\omega_{(p)}^{2}
$$

where the component $\omega_{(p)}^{i}$ is of rank $i$ on the $T^{2}$ fiber. That is,

$$
\begin{aligned}
& \omega_{(p)}^{0}=\frac{1}{p !} \omega_{(p) m^{1} \ldots m^{p}} d x^{m^{1}} \wedge \ldots \wedge d x^{m^{p}} \\
& \omega_{(p)}^{1}=\eta^{\alpha} \wedge \omega_{(p) \alpha}, \quad \omega_{(p)}^{2}=\frac{1}{2} \eta^{\alpha} \wedge \eta^{\beta} \wedge \omega_{(p) \alpha \beta},
\end{aligned}
$$

where

$$
\begin{aligned}
\omega_{(p) \alpha} & =\frac{1}{(p-1) !} \omega_{(p) \alpha m^{2} \ldots m^{p}} d x^{m^{2}} \wedge \ldots \wedge d x^{m^{p}} \\
\omega_{(p) \alpha \beta} & =\frac{1}{(p-2) !} \omega_{(p) \alpha \beta m^{3} \ldots m^{p}} d x^{m^{3}} \wedge \ldots \wedge d x^{m^{p}} .
\end{aligned}
$$

From our restrictions on the flux (including the additional assumption $H_{(3) \alpha \beta m}=0$ made at the end of the last section) we have

$$
F_{(1)}=F_{(1)}^{0}, \quad H_{(3)}=H_{(3)}^{0}, \quad \text { and } \quad \widetilde{F}_{(3)}=\widetilde{F}_{(3)}^{0}+\widetilde{F}_{(3)}^{1}
$$

12 The result of T-dualizing $H_{(3)}$ in the directions of two of its indices is a nongeometrical compactification [54, 44. 
Since the first two decompositions are trivial, we drop the superscripts on $F_{(1)}$ and $H_{(3)}$ everywhere except in App. C, where the assumption $H_{(3) \alpha \beta m}=0$ is temporarily relaxed. We also drop the tilde on $\widetilde{F}_{(3) \alpha}$ since $\widetilde{F}_{(3)}^{1}=F_{(3)}^{1}$ for $H_{(3)}$ even. We will not need to assume anything about $\widetilde{F}_{(5)}$ in order to show in the next section that it must vanish. We note only that $\omega_{(5)}^{0}=0$ identically for any five-form on $X_{6}$. Therefore,

$$
\widetilde{F}_{(5)}=\widetilde{F}_{(5)}^{1}+\widetilde{F}_{(5)}^{2} .
$$

The utility of these definitions is that forms (3.10a) and (3.11) can be interpreted as (pullbacks of) forms on the base. Noting that $d \widetilde{F}_{(3)}^{1}=\mathcal{F}^{\alpha} \wedge F_{(3) \alpha}-\eta^{\alpha} \wedge d F_{(3) \alpha}$, the Bianchi identity (3.7) becomes

$$
d \widetilde{F}_{(3)}^{0}=-\mathcal{F}^{\alpha} \wedge F_{(3) \alpha}-F_{(1)} \wedge H_{(3)}-(2 \pi)^{2} \alpha^{\prime} \operatorname{Vol}_{\mathcal{B}} \sum_{i} Q_{i} \frac{\delta^{4}\left(x-x_{i}\right)}{\sqrt{g_{\mathcal{B}}}}
$$

together with

$$
d F_{(3) \alpha}=0 .
$$

All forms in (3.14a) are now (pullbacks of) forms on the base. So, the entire equation can be integrated over the base to give the Gauss's law constraint

$$
0=N_{\text {flux }}+\sum_{i} Q_{i}
$$

where

$$
N_{\text {flux }}=\frac{1}{(2 \pi)^{2} \alpha^{\prime}} \int_{\mathcal{B}}\left(\mathcal{F}^{\alpha} \wedge F_{(3) \alpha}+F_{(1)} \wedge H_{(3)}\right) .
$$

For the case that the base $\mathcal{B}$ is $T^{4}$,

$$
\sum_{i} Q_{i}=2 M-32
$$

Note that this implies that $N_{\text {flux }}$ must be an even integer. This guaranteed by the Dirac quantization conditions, as will be shown in Sec. 3.9.

The results of this subsection resolve a naive paradox concerning the Bianchi identity. In all but one of the examples that we consider in Secs. 6-8, the only nonzero flux is RR three-form flux and $2 M<32$. Naively, we can integrate both sides of Eq. (3.7) over a transverse four-cycle linking all of the sources of D5 charge, to give zero on the LHS and $(2 M-32)(2 \pi)^{2} \alpha^{\prime}<0$ on the RHS. The resolution is that no such linking cycle exists. 
The desired linking cycle would be a global section embedding the base in the fibration. However, unless the fibration is trivial $\left(\mathcal{F}^{\alpha}=0\right)$, there does not exist such global section. 13 There only exist four-chains $C$ that locally approximate the base in the sense that they project to the base, $\pi C=\mathcal{B}$, but globally necessarily have boundary. Consequently, $\int_{C} d \widetilde{F}_{(3)}=\int_{\partial C} \widetilde{F}_{(3)} \neq 0$. On the other hand, it is true that $\int_{C} d \widetilde{F}_{(3)}^{0}=0$, since this integral is the pullback of $\int_{\mathcal{B}} d \omega_{(3)}$, where $\widetilde{F}_{(3)}^{0}=\pi^{*} \omega_{(3)}$. Note that the nonexistence argument just given only applies to cycles linking all sources of D5 charge. There do exist (homologically trivial) four-cycles linking only individual D5 branes or O5 planes. Therefore, the charge of these objects is still well-defined.

\subsection{Pseudo-BPS Constraints}

As a consequence of the dilaton equation (3.6), the Bianchi identity (3.14a), and the Einstein equation for $g^{\mu \nu} R_{\mu \nu}+g^{\alpha \beta} R_{\alpha \beta}$, it is shown in App. C that

$$
\begin{aligned}
d\left(Z ^ { - 2 } g _ { s } ^ { - 2 } \left(\widetilde{F}_{(3)}^{0}\right.\right. & \left.\left.+Z^{2} *_{\mathcal{B}} d\left(g_{s}^{-1} Z^{-1}\right)\right)\right)= \\
& \frac{1}{2} Z^{-2} g_{s}^{-1}\left(\left(F_{(1)}+*_{\mathcal{B}} g_{s}^{-1} H_{(3)}\right) \wedge *_{\mathcal{B}}\left(F_{(1)}+*_{\mathcal{B}} g_{s}^{-1} H_{(3)}\right)\right. \\
& +2 Z^{-1}\left(\widetilde{F}_{(3)}^{0}+Z^{2} *_{\mathcal{B}} d\left(g_{s}^{-1} Z^{-1}\right)\right) \wedge *_{\mathcal{B}}\left(\widetilde{F}_{(3)}^{0}+Z^{2} *_{\mathcal{B}} d\left(g_{s}^{-1} Z^{-1}\right)\right) \\
& +g_{\alpha \beta}^{\left(T_{\text {fib }}^{2}\right)}\left(F_{(3) \alpha}-g_{\alpha \gamma}^{\left(T_{\text {fib }}^{2}\right)} *_{\mathcal{B}} g_{s}^{-1} \mathcal{F}^{\gamma}\right) \wedge *_{\mathcal{B}}\left(F_{(3) \beta}-g_{\beta \delta}^{\left(T_{\text {fib }}^{2}\right)} *_{\mathcal{B}} g_{s}^{-1} \mathcal{F}^{\delta}\right) \\
& \left.+g_{\alpha \beta}^{\left(T_{\text {fib }}^{2}\right)}\left(2 Z^{-1} \widetilde{F}_{(5) \alpha}^{1} \wedge *_{\mathcal{B}} \widetilde{F}_{(5) \beta}^{1}+g_{\gamma \delta}^{\left(T_{\text {fib }}^{2}\right)} \widetilde{F}_{(5) \alpha \gamma}^{2} \wedge *_{\mathcal{B}} \widetilde{F}_{(5) \beta \delta}^{2}\right)\right) .
\end{aligned}
$$

Here, $g_{s}$ is defined by the equation

$$
e^{\phi}=g_{s} Z^{-1 / 2}
$$

(cf. Eq. (2.1b)) and is not necessarily constant at this point, although that will be shown soon. Since Eq. (3.18) involves only (pullbacks of) forms on the base $\mathcal{B}$, we can integrate both sides over $\mathcal{B}$. The integral of the LHS vanishes, while the RHS is positive semidefinite

13 This statement relies on the particular form of the $T^{2}$ fibration (2.4), in which there are no fiber degenerations associated with the shrinking of a $(p, q) S_{1}$ in the fiber. It is true that there exist, for example, elliptically fibered Calabi-Yau manifolds with global section, but this relies on the existence of such degenerations. 
and vanishes only if all terms vanish individually. Therefore, we obtain the Hodge duality relations

$$
\begin{aligned}
F_{(1)} & =-*_{\mathcal{B}} g_{s}^{-1} H_{(3)}, \\
\widetilde{F}_{(3)}^{0} & =-Z^{2} *_{\mathcal{B}} d\left(g_{s}^{-1} Z^{-1}\right), \\
F_{(3) \alpha} & =g_{\alpha \beta}^{\left(T_{\mathrm{fib}}^{2}\right)} *_{\mathcal{B}} g_{s}^{-1} \mathcal{F}^{\beta}, \\
\widetilde{F}_{(5)} & =0 .
\end{aligned}
$$

We can alternatively write the first three relations as

$$
\begin{aligned}
& * \widetilde{F}_{(3)}=d\left(e^{-\phi} \operatorname{Vol}_{h(x)}\right), \\
& * F_{(1)}=e^{-\phi} \operatorname{Vol}_{h(x)} \wedge H_{(3)},
\end{aligned}
$$

where $*$ is $10 \mathrm{D}$ Hodge star operator in the metric (2.4), and $\operatorname{Vol}_{h(x)}$ is the volume form in the metric (3.5) with the point $x$ on the base allowed to vary:

$$
\operatorname{Vol}_{h(x)}=Z^{-3 / 2} d x^{0123} \wedge \operatorname{Vol}_{\text {fib }}
$$

Here,

$$
d x^{0123}=d x^{0} \wedge d x^{1} \wedge d x^{2} \wedge d x^{3}
$$

and

$$
\mathrm{Vol}_{\mathrm{fib}}=V_{\mathrm{fib}} \eta^{4}(x) \wedge \eta^{5}(x),
$$

with $V_{\text {fib }}$ given by Eq. (2.16). We will refer to the constraints (3.20) and (3.21) as pseudoBPS conditions, since (i) they arise from the equations of motion alone, with no supersymmetry conditions imposed, and (ii) they give a proper subset of the supersymmetry conditions. The existence of such pseudo-BPS conditions is a consequence of the metric ansatz with 4D Minkowski space in the noncompact directions, together with a property of the low energy supergravity theory known as no-scale structure: consistency of the ansatz requires vanishing $4 \mathrm{D}$ vacuum energy, and the no-scale structure implies that the scalar potential is positive semidefinite.14

14 The canonical example of no-scale structure is the $\mathcal{N}=1$ no-scale structure of a theory whose superpotential is independent of Kähler moduli. Then, modulo $D$-terms, the scalar potential is $V=\sum\left|\partial_{i} W\right|^{2}-3|W|^{2}=\sum^{\prime}\left|\partial_{i} W\right|^{2}$, where $\sum$ runs over all moduli and $\sum^{\prime}$ runs over all moduli other than the Kähler moduli. The models of Ref. [9] possess exactly this type of no-scale structure. In the $\mathrm{O} 3$ orientifolds of Refs. [60,11], the no-scale structure is an $\mathcal{N}>1$ analog of this. For the O5 theory discussed here, the only difference is that the O3 Kähler moduli are replaced by a more complicated subset of the O5 moduli. See Refs. [63, 18, 9]. 
Though the pseudo-BPS conditions were derived from the closed string sector equations of motion, they have a clear interpretation in the open string sector. The interpretation of Eq. (3.21a $)$ is that $\mathrm{Vol}_{\text {fib }}$ is a generalized calibration for fiber-class cycles wrapped by the D5 branes. The mass of a D5 brane that wraps a two-cycle in the homology class of the $T^{2}$ fiber is minimized when the two-cycle is the vertical fiber over a point in the base, as opposed to another cycle in the same homology class with horizontal components. For certain choices of flux, such as those in the examples that we present in Secs. 6.1 and 8.1, the S-dual of this calibration condition has already appeared elsewhere [28,27]. The S-duality is discussed briefly in Sec. 4.3. In the earlier treatment, the generalized calibration $\mathrm{Vol}_{\text {fib }}$ was given a further interpretation as deriving from an $\mathrm{SU}(2)$ structure on the 6D internal manifold 15

Eq. (3.216) is the condition that the Myers D5 worldvolume coupling [64]

$$
S_{\Phi^{3}}=-T_{5} \int_{\mathrm{wv}}\left(e^{-\phi} \operatorname{Vol}_{\mathrm{wv}} H_{(3) m n p}-F_{(9) 012345 m n p} d x^{0123} \wedge \eta^{4} \wedge \eta^{5}\right) \operatorname{Tr}\left(\Phi^{m} \Phi^{n} \Phi^{p}\right)
$$

vanish. Here $\mathrm{Vol}_{\mathrm{wv}}$ is the volume form on the D5 worldvolume. When this condition is not satisfied, the D5 branes are polarized by the external $F_{(1)}$ and $H_{(3)}$ fluxes and blow up into D7 branes.

\subsection{Dilaton}

The dilaton equation of motion (3.6) can be combined with the $g^{\mu \nu} R_{\mu \nu}$ Einstein equation to give

$$
\begin{aligned}
-\nabla_{\mathcal{B}}^{2}\left(Z^{-1} e^{-2 \phi}\right) & =Z^{-1 / 2} e^{-2 \phi}\left(g^{\mu \nu}\left(R_{\mu \nu}+2 \nabla_{\mu} \nabla_{\nu} \phi\right)+2\left(\nabla^{2} \phi-2(\nabla \phi)^{2}\right)\right) \\
& =Z^{-1 / 2}\left(F_{(1)}{ }^{2}+\frac{1}{2} \frac{1}{5 !} \widetilde{F}_{(5)}{ }^{2}-e^{-2 \phi} \frac{1}{3 !} H_{(3)}{ }^{2}\right) .
\end{aligned}
$$

(See App. C, Eqs. (C.1) and (C.5)). The RHS vanishes by Eqs. (3.20), so $g_{s}$ as defined in Eq. (3.19) is indeed constant.

15 The $\mathrm{SU}(2)$ structure on $X_{6}$ is in this case defined by a triple of two-forms $J, \operatorname{Re} \Omega_{(2)}$ and $\operatorname{Im} \Omega_{(2)}$ on the $4 \mathrm{D}$ base, together with a pair of Killing one-forms $K_{1}$ and $K_{2}$, such that $K_{1} \wedge K_{2}=$ $\mathrm{Vol}_{\text {fib }}$. 


\subsection{Warp Factor}

The warp factor $Z$ is a solution to Poisson's equation with localized sources at the D5 branes and O5 planes, and constant D5 charge density from the flux and fibration. From the result that $g_{s}$ is constant,

$$
d \widetilde{F}_{(3)}^{0}=\frac{1}{g_{s}} d *_{\mathcal{B}} d Z=\frac{1}{g_{s}} \nabla_{\mathcal{B}}^{2} Z \operatorname{Vol}_{\mathcal{B}} .
$$

It will be shown in Sec 3.7 and 3.8 that $\mathcal{F}^{\alpha}, F_{(3) \alpha}, H_{(3)}$, and $F_{(1)}$ are (pullbacks of) harmonic forms on $\mathcal{B}$ as a consequence of the geometrical conditions on $\mathcal{F}^{\alpha}$ and the Bianchi identities/equations of motion for the fluxes. Therefore, the integrand in Eq. (3.16) is a harmonic representatives of $H^{4}(\mathcal{B}, \mathbb{R})$, that is, a constant multiple of $\operatorname{Vol}_{\mathcal{B}}$. This allows us to replace Eq. (3.16) with the stronger relation

$$
\mathcal{F}^{\alpha} \wedge F_{(3) \alpha}+F_{(1)} \wedge H_{(3)}=(2 \pi)^{2} \alpha^{\prime} N_{\text {flux }} V_{\mathcal{B}}{ }^{-1} \operatorname{Vol}_{\mathcal{B}}
$$

Here, $V_{\mathcal{B}}=\int_{\mathcal{B}} \operatorname{Vol}_{\mathcal{B}}$ is the volume of the base. Note that from Eqs. (3.20a, , we then have $N_{\text {flux }} \geq 0$. Therefore, the number of D5 branes is bounded above by (the absolute value of) the total charge from O5 planes, due to the constraint (3.15).

Combining Eqs. (3.27) and (3.28), the Bianchi identity (3.14a) becomes

$$
\begin{aligned}
-\frac{1}{g_{s}} \nabla_{\mathcal{B}}^{2} Z & =(2 \pi)^{2} \alpha^{\prime}\left(\frac{N_{\text {flux }}}{V_{\mathcal{B}}}+\sum_{i} Q_{i} \frac{\delta^{4}\left(x-x_{i}\right)}{\sqrt{g_{\mathcal{B}}}}\right) \\
& =(2 \pi)^{2} \alpha^{\prime} \sum_{i} Q_{i}\left(\frac{\delta^{4}\left(x-x_{i}\right)}{\sqrt{g_{\mathcal{B}}}}-\frac{1}{V_{\mathcal{B}}}\right) .
\end{aligned}
$$

The solution is

$$
Z=1+(2 \pi)^{2} \alpha^{\prime} g_{s} \sum_{i} Q_{i} G_{\mathcal{B}}\left(x, x_{i}\right),
$$

where $G_{\mathcal{B}}$ is the Green's function for Poisson's equation on the base $\mathcal{B}$,

$$
-\nabla_{\mathcal{B}}^{2} G_{\mathcal{B}}\left(x, x^{\prime}\right)=\frac{\delta^{4}\left(x-x^{\prime}\right)}{\sqrt{g_{\mathcal{B}}}}-\frac{1}{V_{\mathcal{B}}} .
$$

As part of our definition of the Green's function, we fix the constant component of $G_{\mathcal{B}}$ by requiring that $\int_{\mathcal{B}} d^{4} x \sqrt{g_{\mathcal{B}}} G_{\mathcal{B}}\left(x, x^{\prime}\right)=0$. The leading integration constant of unity in Eq. (3.30) is conventional and ensures that the warp factor completely drops out of the metric (2.4) in the limit of zero gravitational coupling $g_{s} \rightarrow 0$, at fixed $V_{\mathcal{B}}$. This fixes the rescaling ambiguity

$$
g_{s} \rightarrow \lambda g_{s}, \quad Z \rightarrow \lambda^{2} Z \quad \text { at fixed } e^{\phi}
$$

in Eq. (3.19). 


\subsection{Base Geometry}

The only other constraint that follows from the Einstein, dilaton, and $\widetilde{F}_{(3)}$ equations, and is not automatically satisfied as a consequence of Eqs. (3.20), (3.26), (3.14b), and $(3.30)$ is

$$
R_{\mathcal{B} m n}=0
$$

from the $m n$ Einstein equations. For compact base $\mathcal{B}$, this comes close to implying that $\mathcal{B}$ is $T^{4}$ or $K 3$. Where it falls short is that Ricci-flatness implies only that $c_{1}(\mathcal{B})$ vanishes in $H^{2}(\mathcal{B}, \mathbb{R})$. There can still be a torsion component in $H^{2}(\mathcal{B}, \mathbb{Z})$, as is the case for an Enriques surface. Such manifolds are ruled out only after we impose supersymmetry. The supersymmetry conditions $\partial_{\alpha} \hat{\chi}=\nabla_{\mathcal{B} m} \hat{\chi}=0$ of Sec. 4.4 give $c_{1}(\mathcal{B})=0$ as an integrability condition.

\subsection{Geometrical Bianchi Identity and Quantization of the Fibration Curvature}

For the fibration (2.4) to be globally well-defined, the fibration curvature must satisfy

$$
d \mathcal{F}^{\alpha}=0
$$

This is equivalent to the triple-overlap condition on the transition functions that relate coordinate patches. Under a fiber coordinate redefinition, $x^{\alpha} \rightarrow x^{\alpha}+\Lambda^{\alpha}\left(\left\{x^{m}\right\}\right)$, we have $\mathcal{F}^{\alpha} \rightarrow \mathcal{F}^{\alpha}-d \Lambda^{\alpha}$ (cf. App. B). Therefore, the exact part of $\mathcal{F}^{\alpha}$ contains coordinate-gauge information, and the topology of the fibration is characterized by the cohomology class of $\mathcal{F}^{\alpha}$.

We require that $\left[\mathcal{F}^{\alpha}\right]$ be $2 \mathbb{Z}$ valued $(\mathrm{cf}$. Eq. (2.5)) for the following reason. First, ignore the orientifold operation. The subgroup of $H^{2}\left(X_{6}, \mathbb{Z}\right)$ that comes directly from $H^{2}(\mathcal{B}, \mathbb{Z})$ is the quotient group $H^{2}(\mathcal{B}, \mathbb{Z}) /\left\{\left[\mathcal{F}^{\alpha}\right]\right\}$. This quotient is well-defined only if $\left[\mathcal{F}^{\alpha}\right] \in H^{2}(\mathcal{B}, \mathbb{Z})$. Then, in order to define the orientifold, we further require that $\mathcal{F}^{\alpha}$ describe a fibration over $\mathcal{B} / \mathbb{Z}_{2}$. This is equivalent to the condition

$$
\left[\mathcal{F}^{\alpha}\right] \in H^{2}(\mathcal{B}, 2 \mathbb{Z})
$$

which guarantees integer periods of $\left[\mathcal{F}^{\alpha}\right]$ over the cycles in $\mathcal{B} / \mathbb{Z}_{2}$ that descend from halfcycles in $\mathcal{B}$. It is possible that the $2 \mathbb{Z}$ quantization condition can be replaced by a $2 \mathbb{Z}+1$ quantization condition if one includes localized fibration curvature at some or all of the $\mathbb{Z}_{2}$ fixed points. However, we do not consider such localized curvature here. 


\subsection{Flux Bianchi Identities/Equations of Motion}

In addition to Eqs. (3.14a,b) on $\widetilde{F}_{(3)}$, the Bianchi identities/equations of motion that the fluxes must satisfy are

$$
d F_{(1)}=0, \quad d \widetilde{F}_{(5)}=0, \quad \text { and } \quad d H_{(3)}=0
$$

together the conditions

$$
\begin{aligned}
d \widetilde{F}_{(9)} & =H_{(3)} \wedge \widetilde{F}_{(7)}, \\
d \widetilde{F}_{(7)} & =H_{(3)} \wedge \widetilde{F}_{(5)}, \\
d\left(e^{-2 \phi} H_{(7)}\right) & =F_{(1)} \wedge \widetilde{F}_{(7)},
\end{aligned}
$$

on the dual fluxes

$$
\widetilde{F}_{(9)}=* F_{(1)}, \quad \widetilde{F}_{(7)}=* \widetilde{F}_{(3)}, \quad \text { and } \quad H_{(7)}=* H_{(3)} .
$$

The middle dimensional flux $\widetilde{F}_{(5)}$ is selfdual, $\widetilde{F}_{(5)}=* \widetilde{F}_{(5)}$. Here, $*$ is the 10D Hodge star operator in the metric (2.4). In writing these equations, we have used the fact that the only local sources in our model are O5 planes and D5 branes.

The conditions on the dual fluxes are automatically satisfied as a consequence of Eq. (3.36) and the Hodge duality constraints (3.20). For example,

$$
\widetilde{F}_{(9)}=* F_{(1)}=-Z^{-1} d x^{0123} \wedge \eta^{4} \wedge \eta^{5} \wedge\left(*_{\mathcal{B}} F_{(1)}\right) .
$$

Using Eqs. (3.19), (3.26), (3.20a), and the Bianchi identity $d H_{(3)}=0$, this becomes

$$
d \widetilde{F}_{(9)}=d\left(e^{-\phi} \operatorname{Vol}_{h(x)}\right) \wedge H_{(3)},
$$

which, via Eq. (3.21a), is the desired Bianchi identity (3.37a) for $\widetilde{F}_{(9)}$. Eqs. (3.37b, d) follow analogously.

Note that Eqs. (3.36) and (3.200) imply that after imposing the equations of motion, $F_{(1)}$ and $H_{(3)}$ are harmonic on $\mathcal{B}$, and $F_{(5) \alpha \beta}$ is closed. Similarly, Eqs. (3.14b), (3.34), and (3.20) imply that $\mathcal{F}^{\alpha}$ and $F_{(3) \alpha}$ are harmonic on $\mathcal{B}$. 


\subsection{Discrete Data versus Moduli, and Additional Moduli Constraints}

Beyond the choice of base manifold $\mathcal{B}$ and orientifold operation, the complete set of discrete data needed to define the model is the number $2 M$ of D5 branes and their images, together with the quantized parts of the even fluxes and the fibration curvature (3.35).

For later discussion of moduli stabilization, it is necessary to decompose the fluxes into a part that is moduli-dependent and a quantized part that only involves the discrete choice. For simplicity of exposition, we restrict to the case that $\mathcal{B}=T^{4}$. (The results of this section are analogous in the case that $\mathcal{B}=K 3$, except that the absence of a first cohomology class for $K 3$ implies that $F_{(1)}=0$, and then from Eq. (3.20a), $H_{(3)}=0$.) In this subsection, we also drop all functional dependence of the moduli on $x^{\mu}$. That is, we analyze deformations of the supergravity background, and do not yet promote these deformations to $4 \mathrm{D}$ fields. We restrict to deformations that correspond to moduli and not to gauge bosons. In the next subsection, when we discuss the kinetic terms and gauge couplings, the $x^{\mu}$-dependence and gauge bosons will be reintroduced.

Let us write all of the internal fluxes and potentials as the sum of a background value and a deformation. In the NS sector we write

$$
\begin{aligned}
& B_{(2)}=B_{(2)}^{\mathrm{bg}}+b_{(2)}, \\
& H_{(3)}=H_{(3)}^{\mathrm{bg}}+h_{(3)} .
\end{aligned}
$$

Since we have assumed that $H_{(3)}=H_{(3)}^{0}$ (in the notation of Sec. 3.2), we take $B_{(2)}^{\mathrm{bg}}$ to have purely base components, $B_{(2)}^{\mathrm{bg}}=B_{(2)}^{\mathrm{bg} 0}$. The deformation permitted by the orientifold projection is a shift $b_{(2) \alpha m}$ in the zero-mode, or constant component, 16 of the even potential $B_{(2) \alpha m}$. Therefore,

$$
\begin{aligned}
b_{(2)} & =\eta^{\alpha} \wedge b_{(2) \alpha}, \\
h_{(3)} & =d b_{(2)}=\mathcal{F}^{\alpha} \wedge b_{(2) \alpha} .
\end{aligned}
$$

In the $\mathrm{RR}$ sector, we similarly write

$$
\begin{aligned}
C_{(p)} & =C_{(p)}^{\mathrm{bg}}+c_{(p)}, \\
F_{(p+1)} & =F_{(p+1)}^{\mathrm{bg}}+f_{(p+1)}, \quad \widetilde{F}_{(p+1)}=\widetilde{F}_{(p+1)}^{\mathrm{bg}}+\widetilde{f}_{(p+1)} .
\end{aligned}
$$

16 To justify this usage of the term zero-mode, note that the $p$-forms with constant coefficients are annihilated by a Laplacian operator on $X_{6}$ formed from a torsionful connection that forgets about the fibration and warping (cf. Sec. 4.4). 
In this case, the moduli are not quite the $c_{(p)}$. The latter are in general multivalued quantities on $X_{6}$ due to the action of the $C_{(p-2)}$ transition functions on $C_{(p)}$ when $H_{(3)} \neq 0$. This subtlety is discussed in Ref. [60]. Instead, the moduli are the combinations

$$
\begin{aligned}
& \widetilde{c}_{(2)}=c_{(2)}-b_{(2)} C_{(0)}^{\mathrm{bg}}, \\
& \widetilde{c}_{(4)}=c_{(4)}-b_{(2)} \wedge\left(C_{(2)}^{\mathrm{bg} 1}+\widetilde{c}_{(2)}\right)-\frac{1}{2} b_{(2)} \wedge b_{(2)} \wedge C_{(0)}^{\mathrm{bg}}, \\
& \widetilde{c}_{(6)}=c_{(6)}-b_{(2)} \wedge \widetilde{c}_{(4)}-\frac{1}{2} b_{(2)} \wedge b_{(2)} \wedge \widetilde{c}_{(2)},
\end{aligned}
$$

as can be verified by arguments analogous to those in Ref. [60]. Here, Eqs. (3.44a,b,c) are relations between quantities with internal 6D indices only.

The nonvanishing components of the background are

$$
C_{(0)}^{\mathrm{bg}}, \quad C_{(2) m n}^{\mathrm{bg}}, \quad C_{(2) \alpha m}^{\mathrm{bg}}, \quad C_{(4) m n p q}^{\mathrm{bg}}, \quad C_{(4) \alpha n p q}^{\mathrm{bg}},
$$

or a subset thereof, and the moduli are the zero-modes

$$
\widetilde{c}_{(2) m n}, \quad \widetilde{c}_{(2) \alpha \beta}, \quad \widetilde{c}_{(4) \alpha m n p}, \quad \widetilde{c}_{(6) \alpha \beta m n p q}
$$

The last modulus is $\widetilde{c}_{(6)} 456789$. This is the scalar that is dual to the corresponding deformation $\widetilde{c}_{(2) \mu \nu}$ of $C_{(2) \mu \nu}$, and will be more convenient to work with than $\widetilde{c}_{\mu \nu}$ when we discuss gauge couplings in Sec. 3.10 and the T-duality map in Sec. 5.2.

The flux deformations that follow from Eqs. (3.44a,b, d) are

$$
\begin{aligned}
& f_{(3)}=\widetilde{f}_{(3)}=d \widetilde{c}_{(2)}+b_{(2)} \wedge F_{(1)}, \\
& \tilde{f}_{(5)}=-\widetilde{c}_{(2)} \wedge H_{(3)}^{\mathrm{bg}}+b_{(2)} \wedge\left(F_{(3)}^{\mathrm{bg} 1}+d \widetilde{c}_{(2)}\right)+\frac{1}{2} b_{(2)} \wedge b_{(2)} \wedge F_{(1)},
\end{aligned}
$$

expressable in terms of the moduli and the gauge invariant fluxes. The second equality in Eq. (3.47a) implies that the only nonvanishing component of $\widetilde{f}_{(3)}$ is $\widetilde{f}_{(3) \alpha}$, with one fiber index; this, combined with the earlier observation that $\widetilde{F}_{(3) \alpha}=F_{(3) \alpha}$, gives the first equality. In Eq. (3.47b), $F_{(1)}=F_{(1)}^{\mathrm{bg}}$, so we have dropped the superscript.

One conseqence of Eqs. (3.42) and (3.47a) is that

$$
\mathcal{F}^{\alpha} \wedge f_{(3) \alpha}+F_{(1)} \wedge h_{(3)}=0
$$

from which Eq. (3.16) becomes

$$
N_{\text {flux }}=\frac{1}{(2 \pi)^{2} \alpha^{\prime}} \int_{\mathcal{B}}\left(\mathcal{F}^{\alpha} \wedge F_{(3) \alpha}^{\mathrm{bg}}+F_{(1)} \wedge H_{(3)}^{\mathrm{bg}}\right),
$$


independent of the moduli.

Dirac quantization constrains the closed moduli-independent fluxes $F_{(1)}, F_{(3) \alpha}^{\mathrm{bg}}, F_{(5) \alpha \beta}^{\mathrm{bg}}$, and $H_{(3)}^{\mathrm{bg}}$ to be representatives of $2 \mathbb{Z}$-valued cohomology:

$$
\begin{aligned}
& {\left[F_{(1)}\right] \in H^{1}(\mathcal{B}, 2 \mathbb{Z}), \quad\left[F_{(3) \alpha}^{\mathrm{bg}}\right] \in(2 \pi)^{2} \alpha^{\prime} H^{2}(\mathcal{B}, 2 \mathbb{Z}),} \\
& {\left[H_{(3)}^{\mathrm{bg}}\right] \in(2 \pi)^{2} \alpha^{\prime} H^{2}(\mathcal{B}, 2 \mathbb{Z}), \quad\left[F_{(5) \alpha \beta}^{\mathrm{bg}}\right] \in(2 \pi)^{4} \alpha^{\prime 2} H^{3}(\mathcal{B}, 2 \mathbb{Z}) .}
\end{aligned}
$$

As in Eq. (3.35), the $2 \mathbb{Z}$ quantization of periods on the covering space $\mathcal{B}$ ensures $\mathbb{Z}$ quantization of periods over half cycles in $\mathcal{B}$ that descend to proper boundaryless cycles in $\mathcal{B} / \mathbb{Z}_{2}$. We can now return to the issue of the even integer quantization of $N_{\text {flux }}$ mentioned in Sec. 3.2. From Eq. (3.49), we see that $N_{\text {flux }}$ depends only on the discrete choice (3.35) and (3.50.) As a result, $N_{\text {flux }}$ satisfies $N_{\text {flux }} \in 4 \mathbb{Z}$, which is indeed an even integer, and is moduli independent, so no moduli constraints arise from the Gauss's law constraint.

While Dirac quantization alone permits a quantized five-form flux $F_{(5) \alpha \beta}^{\mathrm{bg}}$, it can be shown that $F_{(5) \alpha \beta}^{\mathrm{bg}}=0$ in order to satisfy the equations of motion. We omit the direct proof, but Sec. 5.2 contains a proof via T-duality. From the constraint (3.20d), we then have

$$
\widetilde{f}_{(5)}=0
$$

Without imposing any supersymmetry conditions, the complete set of moduli constraints that follow from the equations of motion consists of Eqs. (3.20a,d) and (3.51). When $F_{(1)}=H_{(3)}^{\mathrm{bg}}=0$, the constraints simplify. Eq. (3.20a) then implies that

$$
0=h_{(3)}=\mathcal{F}^{\alpha} \wedge b_{(2) \alpha}
$$

and the constraint (3.51) becomes

$$
b_{(2)[\alpha} \wedge F_{(3) \beta]}^{\mathrm{bg}}=0 .
$$

In this case, the complete set of moduli constraints is (3.20d) and Eqs. $(3.52 a, y)$.

\subsection{Couplings to Gauge Bosons}

In addition to the deformations of the supergravity background just discussed, which when promoted to 4D fields become moduli, there are other deformations of the supergravity background which when promoted to $4 \mathrm{D}$ fields become gauge bosons. In the closed 
string sector, these deformations are the zero modes on $X_{6}$ of $V^{\alpha}{ }_{\mu}, B_{(2) m \mu}, C_{(2) \alpha \mu}$, and $C_{(4) \alpha \beta m \mu}+6 b_{(2)[\alpha m \mid} C_{(2) \mid \beta \mu]}$, and will be denoted by the lowercase symbols

$$
v_{\mu}^{\alpha}, \quad b_{(2) m \mu}, \quad c_{(2) \alpha \mu}, \quad \text { and } \quad \widetilde{c}_{(4) \alpha \beta m \mu}
$$

respectively. When the couplings to these gauge bosons are included and all deformations are promoted to $4 \mathrm{D}$ fields, the kinetic term for $\widetilde{c}_{(2) m n}$ is the square of

$$
\partial_{\mu} \widetilde{c}_{(2) m n}-F_{(3) \alpha m n}^{\mathrm{bg}} v^{\alpha}{ }_{\mu}+2 F_{(1)[m} b_{(2) n] \mu}+\mathcal{F}^{\alpha}{ }_{m n} c_{(2) \alpha \mu},
$$

that for $\widetilde{c}_{(4) \alpha m n p}$ is the square of

$$
\partial_{\mu} \widetilde{c}_{(4) \alpha m n p}+3 \mathcal{F}_{[m n \mid}^{\beta} \widetilde{c}_{(4) \beta \alpha \mid p] \mu}+H_{(3) m n p}^{\mathrm{bg}} c_{(2) \alpha \mu}+3 F^{\mathrm{bg}}{ }_{(3) \alpha[m n} b_{(2) p] \mu},
$$

and that for $\widetilde{c}_{(6) \alpha \beta m n p q}$ (the scalar that is dual to $\widetilde{c}_{(2) \mu \nu}$ ) is the square of

$$
\partial_{\mu} \widetilde{c}_{(6) \alpha \beta m n p q}-3 H_{(3)[m n p \mid}^{\mathrm{bg}} \widetilde{c}_{(4) \alpha \beta \mid q] \mu} .
$$

The axion $\widetilde{c}_{(2) \alpha \beta}$ of $\tau_{\text {dil }}$ does not couple to gauge bosons.

Beyond the closed string sector gauge bosons, there are also gauge bosons that arise from the lowest Kaluza-Klein mode of the D5 worldvolume gauge fields $A_{I \mu}$ on the wrapped $T^{2}$ fiber.

\section{Supersymmetry Conditions}

The 10D type IIB dilatino and gravitino variations are given in App. D. From these fermion variations, we will now determine the conditions on the $6 \mathrm{D}$ geometry and internal flux for unbroken $4 \mathrm{D} \mathcal{N} \geq 1$ supersymmetry.

\subsection{Decomposition of 10D Supersymmetry Parameters}

In 10D, the IIB supersymmety transformations are parametrized by two MajoranaWeyl spinors $\epsilon_{L, R}$ (real and negative chirality in our conventions), which combine to form a single Weyl spinor $\epsilon=\epsilon_{L}+i \epsilon_{R}$. For compactification to $4 \mathrm{D}$, it is desirable to decompose $\epsilon$ into 4D and 6D spinors.17 An arbitrary 10D negative chirality Weyl spinor $\epsilon$ can be written

$$
\epsilon=u \otimes \chi_{1}+u^{*} \otimes \chi_{2}^{*}
$$

17 In this discussion, we follow Graña and Polchinski [6]. 
where $\chi_{1}$ and $\chi_{2}$ are negative chirality $6 \mathrm{D}$ Weyl spinors, and $u$ is a positive chirality $4 \mathrm{D}$ Weyl spinor.18 For each pair $\left(\chi_{1}, \chi_{2}\right)$ such that (4.1) gives vanishing dilatino and gravitino variation for all $u$, we obtain one $4 \mathrm{D} \mathcal{N}=1$ supersymmetry generated by $u$.

Orientifold planes impose additional constraints on the spinors. Two broad classes of constraints are

$$
\begin{aligned}
& \chi_{1}=0 \quad \text { or } \quad \chi_{2}=0 \quad \text { Becker-type or chiral constraints, } \\
& \chi_{2}=e^{i \alpha} \chi_{1}, \quad \alpha=\text { const } \text { Andy-type or Majorana-Weyl constraints. }
\end{aligned}
$$

Becker-type spinors are of definite 6D and 4D chirality, while Andy-type spinors are real up to an overall constant phase. Roughly speaking, Becker-type constraints are associated with $\mathrm{O} 3$ or O7 planes, and Andy-type constraints are associated with O5 or O9 planes or the heterotic theories. More precisely, O3 and O9 constraints require that the spinors be of pure Becker- or Andy-type; O7 and O5 constraints require that the spinors be a particular linear combination of two Becker- or two Andy-type spinors, such that for certain choices of flux we can decompose the supersymmetry algebra into $\mathcal{N}=1$ subalgebras, each generated by a spinor for which one of the two terms in the linear combination vanishes. We will demonstrate this for the $\mathrm{O} 5$ case which is the focus our investigation, and for the O3 case which is needed in Sec. 5.

Independent of the details of the orientifold projection, $\epsilon_{R}$ is a real Majorana-Weyl spinor, and can therefore be written as

$$
\epsilon_{R}=\frac{1}{\sqrt{2}}\left(u \otimes \chi+u^{*} \otimes \chi^{*}\right)
$$

for some $u$ and $\chi$, where the leading factor of $1 / \sqrt{2}$ is for compatibility with the normalization conventions

$$
\epsilon_{L, R}^{\dagger} \epsilon_{L, R}=u^{\dagger} u=\chi^{\dagger} \chi=1
$$

For the O5 planes of interest, the 10D supersymmetries preserved by the orientifold projection are generated by $\epsilon_{L, R}$ such that

$$
\epsilon_{L}=\Gamma_{\mathcal{B}} \epsilon_{R}
$$

Here, $\Gamma_{\mathcal{B}}$ is the chirality operator formed from product of Dirac matrices in the directions transverse to the orientifold planes, normalized so that $\Gamma_{\mathcal{B}}{ }^{2}=1$. Similarly, we define $\Gamma_{\text {fib }}$

18 Note also that for $\epsilon$ and $u$ to be standard anticommuting spinors, $\chi_{1}$ and $\chi_{2}$ must be commuting spinors 30 . 
to be the chirality operator in the two compact directions wrapped by the O5 plane. That is, $\Gamma_{\text {fib }}$ is the chirality operator on the $T^{2}$ fiber, and $\Gamma_{\mathcal{B}}$ is the chirality operator on the base $\mathcal{B}$. Using these chirality operators, we can decompose the $6 \mathrm{D}$ spinor $\chi$ into components of definite fiber and base chirality,

$$
\chi=\chi_{+}+\chi_{-}, \quad \text { where } \quad \gamma_{\mathrm{fib}} \chi_{ \pm}= \pm \chi_{ \pm}, \quad \gamma_{\mathcal{B}} \chi_{ \pm}=\mp \chi_{ \pm}
$$

Here, we have gone from uppercase 10D to lowercase 6D internal Dirac matrices using the relations $\Gamma_{\text {fib }}=1 \otimes \gamma_{\text {fib }}$ and $\Gamma_{\mathcal{B}}=1 \otimes \gamma_{\mathcal{B}}$. (See App. A for a precise statement of our conventions for Dirac matrices, chirality operators, and their decompositions under 10D $\rightarrow 4 \mathrm{D} \times 6 \mathrm{D} \rightarrow 4 \mathrm{D} \times 2 \mathrm{D} \times 4 \mathrm{D})$.

Applying the decomposition (4.6) to Eq. (4.5), we obtain

$$
\epsilon_{L}=\frac{1}{\sqrt{2}}\left(u \otimes \chi_{L}+u^{*} \otimes \chi_{L}^{*}\right)
$$

where

$$
\chi_{L}=\gamma_{\mathcal{B}} \chi=-\chi_{+}+\chi_{-}
$$

The 10D Weyl spinor $\epsilon=\epsilon_{L}+i \epsilon_{R}$ becomes

$$
\epsilon=-e^{-i \pi / 4}\left(\chi_{+} \otimes u+\chi_{+}^{*} \otimes u^{*}\right)+e^{i \pi / 4}\left(\chi_{-} \otimes u+\chi_{-}^{*} \otimes u^{*}\right)
$$

which is the desired linear combination of two Andy-type spinors. In the case that $F_{(1)}=$ $H_{(3)}=0$, we will show in Sec. 4.2 that the supersymmetry conditions on $\chi_{+}$and $\chi_{-}$ decouple from one another, so that the space of $6 \mathrm{D}$ Killing spinors $\chi$ decomposes into a subspace on which $\chi_{-}=0$ and a subspace on which $\chi_{+}=0$. On either subspace, $\epsilon$ is of pure Andy-type.

For spacetime filling O3 planes, the 10D supersymmetries are generated by $\epsilon_{L, R}$ such that

$$
\epsilon_{L}=-i \Gamma^{(6)} \epsilon_{R}
$$

Here, $\Gamma^{(6)}$ is the chirality operator formed from the product of Dirac matrices in the six internal directions transverse to the orientifold planes. Using Eq. (4.3), this becomes Eq. (4.7) with

$$
\chi_{L}=-i \gamma^{(6)} \chi=i \chi
$$

So, the 10D Weyl spinor is

$$
\epsilon=\sqrt{2} i \chi \otimes u
$$


of Becker-type, as claimed.

Although it is not directly relevant to this investigation, it is interesting to note that there exist supergravity backgrounds that are neither Andy-type or Becker type, but rather interpolate between the two [65,44]. These solutions are D3-like in some regions and D5/NS5-like in others. Similarly, there exist dielectric flow solutions, involving D3 branes that become polarized into D5/NS5 branes under radial flow in IIB, or M2 branes that become polarized into M5 branes under radial flow in M theory [66, 67]. In Ref. [67], the technique of "Algebraic Killing spinors" was developed and repeatedly applied as an efficient method for obtaining solutions to the supergravity equations of motion. In the M theory case, these supergravity solutions fit nicely into classification of Ref. [34], which provides a general framework in the language of $G$-structures for describing arbitrary compactifications of $\mathrm{M}$ theory to $\mathbb{R}^{2,1}$ and $A d S_{3}$ [34]. This framework also accomodates the $\mathrm{M}$ theory dual of Refs. 65, 44]. A more restrictive treatment with applications, in particular, to the $\mathrm{M}$ theory lift of IIB pp-wave backrounds has also been given [68]. Finally, recent work has shown that the correct description of the most general $\mathbb{R}^{3,1}$ or $A d S_{5}$ compactifications of type IIB or M theory, should be given in terms of $S U(2)$ structures [41,36, 42, This work has brought us closer to understanding, for example, what the complete supergravity solution for the Polchinski-Strassler background might be, a subject that is currently under investigation [69].

\subsection{Supersymmetry Conditions for RR Three-Form Flux Only}

In the case that there is only $F_{(3)}$ RR flux and nontrivial fibration, the supersymmetry conditions are very similar to those for the heterotic string with NS flux. By substituting the expression (4.9) for $\epsilon$ into the Eqs. (D.7) for the IIB fermion variations, and then demanding that the result vanish, we obtain

$$
\begin{array}{r}
\left(\frac{1}{2} \not \partial \phi \mp \frac{1}{4} e^{\phi} \mathscr{F}_{(3)}\right) u \otimes \chi_{ \pm}=0, \\
\left(\nabla_{M}-\frac{1}{4} \Gamma_{M} \not \partial \phi \pm \frac{1}{4} e^{\phi} \mathscr{F}_{(3) M}\right) u \otimes \chi_{ \pm}=0 .
\end{array}
$$

After performing a Weyl rescaling

$$
d s_{\text {string }}^{2}=e^{\left(\phi-\phi_{0}\right)} \widehat{d s}^{2}, \quad \chi_{ \pm}=e^{\left(\phi-\phi_{0}\right) / 4} \hat{\chi}_{ \pm}, \quad \Gamma^{M}=e^{-\left(\phi-\phi_{0}\right) / 2} \hat{\Gamma}^{M}
$$

where $e^{\phi_{0}}=g_{s}$, these equations become

$$
\begin{gathered}
\left(\frac{1}{2} \hat{\partial \phi}_{\phi} \mp \frac{1}{4} g_{s} \hat{\mathscr{F}}_{(3)}\right) u \otimes \hat{\chi}_{ \pm}=0, \\
\left(\hat{\nabla}_{M} \pm \frac{1}{4} g_{s} \hat{H}_{(3) M}\right) u \otimes \hat{\chi}_{ \pm}=0 .
\end{gathered}
$$


Here, the hatted metric is

$$
\widehat{d s}^{2}=Z^{-1 / 2} e^{-\left(\phi-\phi_{0}\right)}\left(\eta_{\mu \nu} d x^{\mu} d x^{\nu}+d s_{6}^{2}\right) .
$$

Eqs. (4.15) are a doubled version of the equations that formed the starting point for Strominger's "Superstrings with Torsion" analysis [2]. Since the constraints on $\chi_{+}$and $\chi_{-}$are decoupled from one another, we are free to take $\chi=\chi_{+}$or $\chi_{-}$, and set the other spinor to zero. Then the starting points are identical. The results that follow from the same analysis are just Strominger's results, with $H_{(3)} \rightarrow \mp g_{s} F_{(3)}, \chi \rightarrow \hat{\chi}_{ \pm}$, and $\phi \rightarrow-\phi$ compared to Sec. 1.1.19

$$
\begin{aligned}
Z^{-1 / 2} e^{-\left(\phi-\phi_{0}\right)} & =1, \\
e^{-2 \phi} d\left(e^{2 \phi} J_{ \pm}\right) & = \pm *_{6} g_{s} F_{(3)}, \\
d\left(e^{2 \phi} *_{6} J_{ \pm}\right) & =0 \\
d\left(e^{2 \phi} \Omega_{ \pm}\right) & =0,
\end{aligned}
$$

where the $S U(3)_{ \pm}$structures are2

$$
\begin{aligned}
J_{ \pm a}{ }^{b} & =i \hat{\chi}_{ \pm}^{\dagger} \gamma_{a}^{b} \hat{\chi}_{ \pm}, \\
\Omega_{ \pm a b c} & =\hat{\chi}_{ \pm}^{\dagger} \gamma_{a b c} \hat{\chi}_{ \pm}^{*} .
\end{aligned}
$$

Just as for the heterotic string, $e^{2 \phi} \Omega_{ \pm}$is a holomorphic $(3,0)$ form and the Nijenhuis tensor vanishes, so that the complex structure (4.17a) is integrable. Eq. (4.16a) reproduces the relation (3.19) between the dilaton and warp factor. Eqs. (4.17a,b) imply the relations [2, 32]

$$
\begin{aligned}
J_{ \pm a}{ }^{b} J_{ \pm b}{ }^{c} & =-\delta_{a}{ }^{c}, \\
\frac{1}{3 !} J_{ \pm} \wedge J_{ \pm} \wedge J_{ \pm} & =\frac{i}{8} \Omega_{ \pm} \wedge \bar{\Omega}_{ \pm}=\mathrm{Vol}_{6},
\end{aligned}
$$

where $\mathrm{Vol}_{6}$ is the volume form associated with the metric (2.7),

$$
\operatorname{Vol}_{6}=Z^{2}\left(g^{\left(T_{\text {fib }}^{2}\right)} g_{\mathcal{B}}\right)^{1 / 2} \eta^{4} \wedge \eta^{5} \wedge d x^{6} \wedge d x^{7} \wedge d x^{8} \wedge d x^{9} .
$$

19 The heterotic analogs of Eqs. (4.16a,d $)$ and $4.17 b$ ) were suppressed from our short summary in Sec. 1.1, but follow from Strominger's analysis [2,28,27].

20 The fact that $\Omega_{ \pm}$depends antiholomorphically on $\hat{\chi}_{ \pm}$is a consequence of the negative $\gamma^{(6)}$ chirality of $\hat{\chi}_{ \pm}$. 
In the next two paragraphs, we derive a number of results concerning the fiber and base decomposition of $J_{ \pm}$and $\Omega_{ \pm}$that will be useful for the examples in Sec. 6-8. These paragraphs can be skipped over, if desired, without much loss to the understanding of this section.

Since $\hat{\chi}_{+}$and $\hat{\chi}_{-}$have definite fiber and base chirality, these spinors factorize into the product of a $2 \mathrm{D}$ Weyl spinor on the fiber and a $4 \mathrm{D}$ Weyl spinor on the base,

$$
\hat{\chi}_{ \pm}=\zeta_{ \pm}^{\mathrm{fib}} \otimes \zeta_{ \pm}^{\mathcal{B}}, \quad \text { where } \quad \widetilde{\gamma}_{\mathrm{fib}} \zeta_{ \pm}^{\mathrm{fib}}= \pm \zeta_{ \pm}^{\mathrm{fib}}, \quad \widetilde{\gamma}_{\mathcal{B}} \zeta_{ \pm}^{\mathcal{B}}=\mp \zeta_{ \pm}^{\mathcal{B}}
$$

(The tilded Dirac matrices are defined in App. A). Consequently, the Kähler form and $(3,0)$ form decompose as

$$
J_{ \pm}=J_{ \pm}^{\left(T_{\mathrm{fib}}^{2}\right)}+Z J_{ \pm}^{\mathcal{B}}, \quad \Omega_{ \pm}=\left(2 g_{1 \overline{1}}^{\left(T_{\mathrm{fib}}^{2}\right)}\right)^{1 / 2} \eta^{z^{1}} \wedge Z \Omega_{ \pm}^{\mathcal{B}}
$$

Here, the forms $J_{ \pm}^{\mathcal{B}}$ and $\Omega_{ \pm}^{\mathcal{B}}$ are the Kähler form and holomorphic $(2,0)$ form on the base, defined via

$$
J_{ \pm m}^{\mathcal{B}}{ }^{n}=i \zeta^{\mathcal{B}}{ }_{ \pm}^{\dagger} \widetilde{\gamma}_{m}{ }^{n} \zeta_{ \pm}^{\mathcal{B}}, \quad \Omega_{ \pm m n}^{\mathcal{B}}=\zeta_{ \pm}^{\mathcal{B}}{ }^{\dagger} \widetilde{\gamma}_{m n} \zeta_{ \pm}^{\mathcal{B} *}
$$

Also, in complex coordinates, the fiber metric $(2.4 b)$ is

$$
d s_{T_{\mathrm{fib}}^{2}}^{2}=g_{1 \overline{1}}^{\left(T_{\mathrm{fib}}^{2}\right)} \eta^{z^{1}} \eta^{\bar{z}^{1}}+g_{\overline{1} 1}^{\left(T_{\mathrm{fib}}^{2}\right)} \eta^{\bar{z}^{1}} \eta^{z^{1}}, \quad g_{1 \overline{1}}^{\left(T_{\mathrm{fib}}^{2}\right)}=g_{\overline{1} 1}^{\left(T_{\mathrm{fib}}^{2}\right)}=\frac{V_{\mathrm{fib}}}{2\left|\operatorname{Im} \tau_{1}\right|}
$$

where $V_{\text {fib }}$ is given by Eq. (2.16), and the fiber $(1,0)$ form is

$$
\eta^{z^{1}}=d z^{1}+\mathcal{A}^{z^{1}}, \quad \text { where } \quad z^{1}=x^{4}+\tau_{1} x^{5}, \quad \mathcal{A}^{z^{1}}=\mathcal{A}^{4}+\tau_{1} \mathcal{A}^{5}
$$

in terms of the fiber complex structure modulus $\tau_{1}$.

The fiber complex structure is related to the spinors by

$$
J_{ \pm \alpha}^{\left(T_{\mathrm{fib}}^{2}\right) \beta}=i \zeta_{ \pm}^{\mathrm{fib}}{ }^{\dagger} \widetilde{\gamma}_{\alpha}^{\beta} \zeta_{ \pm}^{\mathrm{fib}}
$$

which implies that

$$
J_{ \pm}^{\left(T_{\mathrm{fib}}^{2}\right)}= \pm \mathrm{Vol}_{\mathrm{fib}}
$$

where $\mathrm{Vol}_{\text {fib }}$ was defined in Eq. (3.24), and

$$
\pm=\operatorname{sign}\left(\operatorname{Im} \tau_{1}\right)
$$


Then, from Eqs. (4.18b) and (4.21),

$$
\frac{1}{2} J_{ \pm}^{\mathcal{B}} \wedge J_{ \pm}^{\mathcal{B}}= \pm \operatorname{Vol}_{\mathcal{B}}
$$

So, the geometrical origin of the \pm doubling in Eqs. (4.16) and (4.17) is that positive orientation of $X_{6}$ (4.18b) can correspond to either positive-positive or negative-negative orientation of the fiber and base (4.26a, $)$.

As a consequence of Eqs. (4.16a) and (4.17b), Eq. (4.16d) is automatically satisfied. The remaining conditions (4.16b, $)$ are equivalent to [29,48

$$
\widetilde{F}_{(3)} \mp \frac{i}{g_{s}} e^{-2 \phi} d\left(e^{2 \phi} J_{ \pm}\right) \quad(2,1) \text { and primitive. }
$$

Using Eqs. (4.21) and (4.26a), and the decomposition (3.12), this becomes

$$
\begin{aligned}
& \widetilde{F}_{(3)}^{0}=*_{\mathcal{B}} g_{s}^{-1} d Z, \\
& G_{(3)} \quad(2,1) \text { and primitive, }
\end{aligned}
$$

where

$$
G_{(3)}=F_{(3)}^{1}-\frac{i}{g_{s}} d \operatorname{Vol}_{\mathrm{fib}} .
$$

From the results of Sec. 3.9 applied to the case that $H_{(3)}=0$, we can also write $G_{(3)}$ as

$$
G_{(3)}=F_{(3)}^{\mathrm{bg} 1}-(2 \pi)^{2} \alpha^{\prime} \tau_{\mathrm{dil}} d\left(\eta^{4} \wedge \eta^{5}\right)
$$

where

$$
\tau_{\text {dil }}=\frac{1}{(2 \pi)^{2} \alpha^{\prime}}\left(-\widetilde{c}_{(2) 45}+\frac{i}{g_{s}} V_{\text {fib }}\right)
$$

which is the suitable generalization of Eq. (2.15) to the case of nonvanishing background flux.

Eqs. (4.28a,b,d) express the supersymmetry conditions in the form that we will find most convenient to apply in Secs. 6-8. Given a discrete choice of $F_{(3) \alpha}^{\mathrm{bg}}$ and $\left[\mathcal{F}^{\alpha}\right]$, we can easily deduce the constraints on moduli via these equations. The number $\mathcal{N}$ of supersymmetries preserved is

$$
\mathcal{N}=\mathcal{N}_{+}+\mathcal{N}_{-}
$$


where $\mathcal{N}_{ \pm}$is the number of independent \pm complex structures (i.e., the number of $\hat{\chi}_{ \pm}$) such that these conditions are satisfied. Eqs. (4.28) also imply the equations of motion (3.20).21 The maximum amount of supersymmetry is $\mathcal{N}=4, \mathcal{N}_{+}=\mathcal{N}_{-}=2$, since there are two $4 \mathrm{D}$ spinors of each chirality and one 2D spinor of each chirality, from which we must construct the negative chirality $6 \mathrm{D}$ spinors $\hat{\chi}_{ \pm}$. For nonvanishing flux, the supersymmetry is strictly less than this. That is, either $\mathcal{N}_{+}<2$ and $\mathcal{N}_{-} \leq 2$, or $\mathcal{N}_{+} \leq 2$ or $\mathcal{N}_{-}<2$.

\subsection{S-dual Orientifolds with NS Flux Only}

The class of supersymmetric vacua just discussed is S-dual to a class of vacua with NS flux $H_{(3)}$ only. Such vacua have received considerable attention, so it is desirable to state the precise connection between Sec. 4.2 and known results.

The result of applying S-duality to the class of orientifolds analyzed here is a class of dual orientifolds in which O5 planes are replaced by ON5 planes and D5 branes are replaced by NS5 planes [70]. The orientifold operation $\Omega \mathcal{I}_{4}$ in the original theory becomes $(-1)^{F_{L}} \mathcal{I}_{4}$ in the dual theory. In our S-duality conventions, the dual NS flux is related to the original RR three-form flux by

$$
H_{(3)}^{\prime}=-F_{(3)}
$$

The S-dual metric is

$$
\begin{aligned}
d s_{\text {string }}^{2} & =e^{-\left(\phi-\phi_{0}\right)} d s_{\text {string }}^{2} \\
& =\eta_{\mu \nu} d x^{\mu} d x^{\nu}+d s_{6}{ }^{2},
\end{aligned}
$$

where we have used Eq. (3.19) (or equivalently, Eq. (4.16a)) in the second equality. We recognize this as the hatted metric 4.150 ) that appeared in the previous section.

The S-dual dilaton is given by

$$
e^{\left(\phi^{\prime}-\phi_{0}^{\prime}\right)}=e^{-\left(\phi-\phi_{0}\right)}=Z^{1 / 2}, \quad g_{s}^{\prime}=g_{s}^{-1}
$$

So, the $6 \mathrm{D}$ metric can be written

$$
d s_{6}{ }^{2}=g_{\alpha \beta}^{\left(T_{\mathrm{fib}}^{2}\right)}\left(d x^{\alpha}+\mathcal{A}^{\alpha}\right)\left(d x^{\beta}+\mathcal{A}^{\beta}\right)+e^{2\left(\phi^{\prime}-\phi_{0}^{\prime}\right)} g_{\mathcal{B} m n} d x^{m} d x^{n}
$$

21 The equations of motion (3.20b,c) require that $G_{3}$ be imaginary-selfdual (ISD): $*{ }_{6} G_{(3)}=$ $i G_{(3)}$. This is weaker than (4.28b). The space of ISD three-forms includes not only primitive $(2,1)$ forms, but also $(0,3)$ forms and non-primitive $(1,2)$ forms $J \wedge \omega$, where $\omega$ is a $(0,1)$ form. 
which is a form that has appeared previously in the literature. Nearly identical vacua were described by Gauntlett, Martelli, and Waldram, in their classification of static supersymmetric backgrounds with NS flux only [27]. The one minor difference is that the local ON5 and NS5 sources were excluded from their discussion, and as a result, the base geometry was required to be noncompact to avoid contradicting the Gauss's law constraint (3.15).

In the conventions of Eq. (4.31), the 10D IIB supersymmetry parameters after the S-duality are

$$
\begin{aligned}
\epsilon_{L}^{\prime} & =\frac{1}{\sqrt{2}}\left(u \otimes \chi_{-}+u^{*} \otimes \chi_{-}^{*}\right), \\
\epsilon_{R}^{\prime} & =\frac{1}{\sqrt{2}}\left(u \otimes \chi_{+}+u^{*} \otimes \chi_{+}^{*}\right),
\end{aligned}
$$

up to a possible overall sign in $\epsilon_{L}^{\prime}$ and/or $\epsilon_{R}^{\prime}$ that will not concern us here. Thus, $\mathcal{N}_{+}$and $\mathcal{N}_{-}$, which before the S-duality counted the number of $6 \mathrm{D}$ Killing spinors of + and - fiber chirality, map to the number of right and left Killing spinors, respectively, in the S-dual theory:

$$
\mathcal{N}_{R}^{\prime}=\mathcal{N}_{+}, \quad \mathcal{N}_{L}^{\prime}=\mathcal{N}_{-} .
$$

In Ref. [27, the metric (4.34) was shown to describe supergravity backgrounds (with $S U(2)$ structure in $6 \mathrm{D})$ such that $\mathcal{N}_{R}^{\prime}>0$ and $\mathcal{N}_{L}^{\prime}=0$. On the other hand, for $\mathcal{N}_{R}^{\prime}=$ $\mathcal{N}_{L}^{\prime}=1$, the metric was shown to possess an almost product structure and take the form

$$
d s_{6}^{\prime 2}=g_{a b}^{(4)} d x^{a} d x^{b}+e^{2\left(\phi-\phi_{0}\right)} g_{c d}^{(2)} d x^{c} d x^{d}, \quad a, b=4,5,6,7, \quad c, d=8,9 .
$$

Here, $g_{a b}^{(4)}$ and $\phi$ can depend on all six coordinates, but $g_{c d}^{(2)}$ can only depend on $x^{8}$ and $x^{9}$.

In contrast, for the orientifold backgrounds studied here, we have argued that the Sdual metric is of the form (4.34), without reference to $\mathcal{N}_{ \pm}$. For the two results to agree, it must be true that for $\mathcal{N}_{+}, \mathcal{N}_{-} \geq 1$, the $6 \mathrm{D}$ metric of the $\mathrm{O} 5$ theory takes a form compatible with both (4.34) and (4.37). In Sec. 6.2, we present an example with $\mathcal{N}_{+}=\mathcal{N}_{-}=1$, in which this is indeed the case. In this example, the base metric $g_{\mathcal{B} m n}$ reduces to the product metric on flat $T_{\{67\}}^{2}$ times flat $T_{\{89\}}^{2}$, and the fibration is such that we can take

$$
\mathcal{A}^{4}=-2 n x^{8} d x^{6}, \quad \mathcal{A}^{5}=-2 n x^{8} d x^{7} .
$$

Also, as is true throughout our investigation, $g_{\alpha \beta}^{\left(T_{\text {fib }}^{2}\right)}$ is constant and the dilaton depends only on the base coordinates. Therefore, the metric (4.34) can be cast in the form (4.37) by writing

$$
\begin{aligned}
& g_{a b}^{(4)} d x^{a} d x^{b}=g_{\alpha \beta}^{\left(T_{\text {fib }}^{2}\right)}\left(d x^{\alpha}+\mathcal{A}^{\alpha}\right)\left(d x^{\alpha}+\mathcal{A}^{\alpha}\right)+e^{2\left(\phi^{\prime}-\phi_{0}^{\prime}\right)} d s_{T^{2}\{67\}}^{2}, \\
& g_{c d}^{(2)} d x^{c} d x^{d}=d s_{T^{2}\{89\}}^{2} .
\end{aligned}
$$

Similar remarks apply to the example in Sec. 7.1, which preserves $\mathcal{N}_{+}=1, \mathcal{N}_{-}=2$ supersymmetry. 


\subsection{Supersymmetry Conditions for More General Flux}

The generalization of Eqs. (4.15a,b) to the case of arbitrary flux (2.10) and (2.11) is

$$
\begin{aligned}
& \left(\frac{1}{2} \hat{\phi} \phi \mp \frac{1}{4} g_{s} \hat{\widetilde{F}}_{(3)}\right) u \otimes \hat{\chi}_{ \pm}+\left(\frac{1}{4} e^{-\left(\phi-\phi_{0}\right)} \hat{H}_{(3)} \mp \frac{1}{2} g_{s} e^{\left(\phi-\phi_{0}\right)} \hat{\mathscr{F}}_{(1)}\right) u \otimes \hat{\chi}_{\mp}=0, \\
& \left(\hat{\nabla}_{M} \pm \frac{1}{4} g_{s} \hat{\mathscr{F}}_{(3) M}\right) u \otimes \hat{\chi}_{ \pm}-\left(\frac{1}{8} e^{-\left(\phi-\phi_{0}\right)}\left(\hat{H}_{(3)} \hat{\Gamma}_{M}+2 \hat{\Gamma}_{M} \hat{H}_{(3)}\right)\right. \\
& \left.\quad \pm \frac{1}{8} g_{s} e^{\left(\phi-\phi_{0}\right)}\left(\hat{H}_{(1)} \hat{\Gamma}_{M}+2 \hat{\Gamma}_{M} \hat{F}_{(1)}\right) \mp \frac{1}{16} g_{s} e^{-\left(\phi-\phi_{0}\right)} \hat{\widetilde{F}}_{(5)} \hat{\Gamma}_{M}\right) u \otimes \hat{\chi}_{\mp}=0 .
\end{aligned}
$$

In analyzing the equations of motion in Sec. 3, we made the simplifying assumption that $H_{(3) \alpha \beta m}=0$ and found that $\widetilde{F}_{(5)}=0$ as a result. It can be shown that the same conclusion also follows if instead of using the equations of motion we impose the supersymmetry conditions plus Bianchi identities.22 However, in this section, we will simply take as a starting point that both $H_{(3) \alpha \beta m}$ and $\widetilde{F}_{(5)}$ vanish.

Since the analysis below is somewhat involved, let us first summarize the results. We will find that the supersymmetry conditions become a refinement of the Hodge duality conditions (3.20a,b, $)$, together with the condition that $\hat{\chi}=\hat{\chi}_{+}+\hat{\chi}_{-}$is constant on the fiber and base,

$$
\partial_{\alpha} \hat{\chi}=0, \quad \nabla_{\mathcal{B} m} \hat{\chi}=0 .
$$

From the internal 6D point of view, Eq. (4.41) says that $\hat{\chi}$ is covariantly constant with respect to a torsionful connection that simply forgets about the warping $(Z)$ and fibration $(\mathcal{A})$ in the metric (2.7). The refinement of Eqs. $(3.20 a, b, b)$ is

$$
\begin{array}{ll}
G_{(1)} & \text { of type }(0,1), \\
\widetilde{G}_{(3)} & (2,1) \text { and primitive, }
\end{array}
$$

where

$$
\begin{aligned}
& \left.G_{(1)}=F_{(1)}-\frac{i}{2}(Z J \wedge J)\right\lrcorner\left(\frac{1}{g_{s}} \operatorname{Vol}_{\text {fib }} \wedge H_{(3)}\right), \\
& \widetilde{G}_{(3)}=G_{(3)}-\frac{i}{2} Z^{-1 / 2} J \wedge G_{(1)},
\end{aligned}
$$

22 When there is maximal unbroken supersymmetry, the supersymmetry conditions together with the Bianchi identities imply the equations of motion. For reduced supersymmetry, this is not necessarily the case, nevertheless, it does seem to be true for the backgrounds presented in [9,27, 11, 4], as well as those presented here. See Ref. [33] for a recent discussion. 
and $G_{(3)}$ is given by Eqs. (4.28d).23 Here, the symbol $\lrcorner$ denotes contraction,

$$
\left.\left(A_{(p)}\right\lrcorner B_{(q)}\right)_{a_{1} \ldots a_{q-p}}=\frac{1}{p !} A_{(p)}{ }^{b_{1} \ldots b_{p}} B_{(q) b_{1} \ldots b_{p} a_{1} \ldots a_{q-p}} .
$$

To obtain these results, first note that Eqs. (4.40) can be further decomposed based on fiber and base chirality. Using the assumption that $H_{(3) \alpha \beta m}$ and $\widetilde{F}_{(5)}$ vanish, the dilatino equation $(4.40 a)$ becomes

$$
\begin{aligned}
& \left(\frac{1}{2} \gamma^{m} \partial_{m} \phi \mp \frac{1}{24} g_{s} \widetilde{F}_{(3) m n p} \gamma^{m n p}\right) \hat{\chi}_{ \pm}=0 \\
& \left( \pm \frac{1}{8} g_{s} F_{(3) \alpha m n} \gamma^{\alpha m n}\right) \hat{\chi}_{ \pm}+\left(\mp \frac{1}{2} g_{s} e^{\left(\phi-\phi_{0}\right)} F_{(1) m} \gamma^{m}+\frac{1}{24} e^{-\left(\phi-\phi_{0}\right)} H_{(3) m n p} \gamma^{m n p}\right) \hat{\chi}_{\mp}=0 .
\end{aligned}
$$

Eq. (4.40b) can be similarly decomposed. In the $M=\mu$ equation, we can take the 4D spacetime spinor $u$ to be constant on $\mathbb{R}^{3,1}$; that is, $\partial_{\mu} u=0$. (If the theory has $4 \mathrm{D}$ local supersymmetry, then it also has 4D global Poincaré supersymmetry). Then, this equation becomes

$$
\begin{aligned}
& \gamma_{\mu}\left(\nabla_{\mathcal{B} m}\left(\phi+\frac{1}{2} \log Z\right)\right) \gamma^{m} \hat{\chi}_{ \pm}=0 \\
& -\frac{1}{8} \gamma_{\mu}\left(\frac{1}{3 !} e^{-\left(\phi-\phi_{0}\right)} H_{(3) m n p} \gamma^{m n p} \mp g_{s} e^{\left(\phi-\phi_{0}\right)} F_{(1) m} \gamma^{m}\right) \hat{\chi}_{\mp}=0 .
\end{aligned}
$$

Here, the leading $\gamma_{\mu}$ factors can be eliminated by contracting with $\frac{1}{4} \gamma^{\mu}$. Eq. (4.45a) is due to the spin connection $\left(\hat{w}_{\mu}\right)_{\nu m}$ in the metric (4.15d). It reproduces the condition that $g_{s}$ as defined in Eq. (3.19) is constant, and it is equivalent to Eq. (4.16a), which states that the prefactor $Z^{-1 / 2} e^{-\left(\phi-\phi_{0}\right)}$ in the hatted metric $(4.158)$ is unity.

For $M=\alpha$, Eq. (4.40b) decomposes into $\partial_{\alpha} \hat{\chi}_{ \pm}=0$ and

$$
-\frac{1}{8}\left(g_{\alpha \beta}^{\left(T_{\mathrm{fib}}^{2}\right)} \mathcal{F}_{m n}^{\beta} \mp g_{s} F_{(3) \alpha m n}\right) \gamma^{m n} \hat{\chi}_{ \pm}=0
$$

as a consequence of Eqs. (4.16a) and (4.45b).

23 In case the reader is bothered by the explicit appearance of the warp factor $Z$ in Eqs. (4.42c,d), we note that $J$ and $\lrcorner$ also contain implicit $Z$ dependence, to that $Z$ drops out of the moduli constraints implied by conditions (4.42a, b) (cf. Sec. 6.3). We can also remove explicit reference to $Z$ from Eqs. (4.42c,d), if desired, by redefining $J$ and $\lrcorner$ in terms of the rescaled metric $Z^{-1 / 2} d s_{6}{ }^{2}$, which is the restriction of the $10 \mathrm{D}$ string frame metric (2.4) to the internal space. 
Finally, using Eqs. (4.16a), (4.44a), and (4.45b), the $M=n$ equation (4.40b) decomposes into $\nabla_{\mathcal{B} n} \hat{\chi}_{ \pm}=0$ and

$$
-\frac{1}{4}\left(g_{\alpha \beta}^{\left(T_{\mathrm{fib}}^{2}\right)} \mathcal{F}_{m n}^{\beta} \mp g_{s} F_{(3) n m \alpha}\right) \gamma^{m \alpha} \hat{\chi}_{ \pm}-\left(\frac{1}{8} e^{-\left(\phi-\phi_{0}\right)} H_{(3) n m p} \gamma^{m p} \mp \frac{1}{4} g_{s} e^{\left(\phi-\phi_{0}\right)} F_{(1) n}\right) \hat{\chi}_{\mp}=0 .
$$

In Sec. 3, we found that the equations of motion and Bianchi identities implied a set of Hodge duality constraints (3.20). We can now reproduce two of these constraints from the supersymmetry conditions as follows. For any Hodge dual pair of odd degree forms on $\mathcal{B}$,

$$
\omega_{(3)}=*_{\mathcal{B}} \omega_{(1)}, \quad \omega_{(1)}=-*_{\mathcal{B}} \omega_{(3)},
$$

we have

$$
\begin{aligned}
\frac{1}{6} \omega_{(3) m n p} \gamma^{m n p} \hat{\chi}_{ \pm} & =\mp Z^{-1} \omega_{(1) r} \gamma^{r} \hat{\chi}_{ \pm} \\
\frac{1}{2} \omega_{(3) m n p} \gamma^{n p} \hat{\chi}_{ \pm} & = \pm Z^{-1} \omega_{(1) r} \gamma^{r}{ }_{n} \hat{\chi}_{ \pm} .
\end{aligned}
$$

From Eq. (4.16a) and the first of these identities, Eqs. (4.44d) and (4.45b) become

$$
\begin{aligned}
& \left(d Z+g_{s} *_{\mathcal{B}} \widetilde{F}_{(3)}^{0}\right)_{m} \gamma^{m} \hat{\chi}_{ \pm}=0 \\
& \left(g_{s} F_{(1)}+*_{\mathcal{B}} H_{(3)}\right)_{m} \gamma^{m} \hat{\chi}_{ \pm}=0
\end{aligned}
$$

Therefore,

$$
\begin{aligned}
d Z & =-*_{\mathcal{B}} g_{s} \widetilde{F}_{(3)}^{0}, \\
g_{s} F_{(1)} & =-*_{\mathcal{B}} H_{(3)},
\end{aligned}
$$

which are the first two constraints $(3.20 a, b)$.

To reproduce the third constraint and obtain the refining primitivity condition, we will need to introduce $S U(3)_{L, R}$ structures. The three spinor conditions that remain are Eqs. (4.44b), (4.46), and (4.47). Using Eqs. (4.16a), (4.49a), and (4.51b), the first of these spinor conditions is implied by the other two. So, Eqs. (4.46) and (4.47) are the complete set of equations that remain. Since the latter couples $\chi_{+}$and $\chi_{-}$, we cannot in general expect to find solutions with $\chi_{+}$or $\chi_{-}$set to zero. Therefore, we will write these constraints in terms of the spinor

$$
\hat{\chi}=e^{\left(\phi-\phi_{0}\right) / 4} \chi=\hat{\chi}_{+}+\hat{\chi}_{-}
$$


(related to $\epsilon_{R}$ by Eq. (4.3)), and the $S U(3)_{R}$ structures constructed from this spinor,

$$
\begin{aligned}
J_{a}{ }^{b} & =i \hat{\chi}^{\dagger} \gamma_{a}{ }^{b} \hat{\chi}, \\
\Omega_{a b c} & =\hat{\chi}^{\dagger} \gamma_{a b c} \hat{\chi}^{*} .
\end{aligned}
$$

Alternatively, we could express the constraints in terms of

$$
\hat{\chi}_{L}=e^{\left(\phi-\phi_{0}\right) / 4} \chi_{L}=-\hat{\chi}_{+}+\hat{\chi}_{-}
$$

(cf. Eqs (4.7) and (4.8)), and the corresponding $S U(3)_{L}$ structures

$$
\begin{aligned}
J_{L a}{ }^{b} & =i \hat{\chi}_{L}^{\dagger} \gamma_{a}{ }^{b} \hat{\chi}_{L}, \\
\Omega_{L a b c} & =\hat{\chi}_{L}^{\dagger} \gamma_{a b c} \hat{\chi}_{L}^{*} .
\end{aligned}
$$

However, since $\chi_{L}$ is related to $\chi$ by Eq. (4.8), the two $\mathrm{SU}(3)$ structures are also related, so that there is no new information gained in doing this.24 Note that in the case that $\chi_{+}$or $\chi_{-}$vanishes, we have $\chi$ and $\chi_{L}$ equal up to a sign, and the $S U(3)_{R}$ and $S U(3)_{L}$ structures are identical. This was the case in Sec. 4.2.

Using Eqs. (4.16a), and the identities (4.49), the two remaining equations become 25

$$
\begin{aligned}
& \left(*_{6} F_{(3)}-\frac{1}{g_{s}} d \mathrm{Vol}_{\mathrm{fib}}\right)_{\alpha m n} \gamma^{m n} \hat{\chi}=0 \\
& \left(\left(F_{(3)}+\frac{i}{g_{s}} d \mathrm{Vol}_{\mathrm{fib}}\right)_{n m \alpha} \gamma^{m \alpha}+\left(Z^{-1 / 2} F_{(1) n}-\frac{1}{g_{s}} Z^{1 / 2}\left(*_{6}\left(\operatorname{Vol}_{\mathrm{fib}} \wedge H_{3}\right)\right)_{m} \gamma^{m}{ }_{n}\right)\right) \hat{\chi}=0 .
\end{aligned}
$$

With the sign conventions

$$
J_{j \bar{k}}=\left(i g_{6}\right)_{j \bar{k}}
$$

(equivalent to Eq. (A.3b)), the nonzero components of the ACS are

$$
J_{j}{ }^{k}=i \delta_{j}{ }^{k}, \quad J_{\bar{\jmath}}{ }^{\bar{k}}=-i \delta_{\bar{\jmath}}{ }^{\bar{k}} .
$$

Together with Eq. (4.53a), this implies that $\hat{\chi}$ is annihilated by $\gamma_{j}$ and $\gamma^{\bar{k}}$, while $\gamma_{\bar{\jmath}}$ and $\gamma^{k}$ act as creation operators. The spinors $\hat{\chi}, \gamma^{\bar{\imath}} \hat{\chi}^{*}$ form a basis for the space of $6 \mathrm{D}$ spinors

24 One can easily show that, for example, $J_{L m n}=J_{m n}, J_{L \alpha \beta}=J_{\alpha \beta}$, and $J_{L m \alpha}=-J_{m \alpha}$.

25 For $\chi \rightarrow \chi_{L}$, the sign of the second term in Eq. (4.56b) is reversed. 
of negative chirality, and $\hat{\chi}^{*}, \gamma^{i} \hat{\chi}$ form a basis for positive chirality $6 \mathrm{D}$ spinors. Using Eq. (4.53b) as well, it is then possible to prove the identities 26

$$
\begin{aligned}
\gamma^{b c} \hat{\chi} & =-\frac{1}{2} \bar{\Omega}^{b c} \gamma^{d} \hat{\chi}^{*}-i J^{b c} \hat{\chi} \\
\gamma^{a} \gamma^{b c} & =-\bar{\Omega}^{a b c} \hat{\chi}^{*}+\left(\frac{1}{4} \bar{\Omega}_{d}^{b c} \Omega_{f}^{d a}-i J^{b c} \delta_{f}^{a}\right) \gamma^{f} \hat{\chi}
\end{aligned}
$$

The latter implies that for an arbitrary three-form $\omega_{(3)}$,

$$
\left.\left.\frac{1}{3 !} \omega_{(3) a b c} \gamma^{a b c} \hat{\chi}=-(\bar{\Omega}\lrcorner \omega_{(3)}\right) \hat{\chi}^{*}-i(J\lrcorner \omega_{(3)}\right)_{i} \gamma^{i} \hat{\chi}
$$

where the contraction operater $\lrcorner$ was defined in Eq. (4.43).

Now, define $G_{(3)}$ as in Eq. (4.28d), and define the complex one-form flux

$$
G_{(1) a}=F_{(1) a}-\frac{i}{g_{s}} Z\left(*_{6}\left(\operatorname{Vol}_{\mathrm{fib}} \wedge H_{(3)}\right)\right)_{b} J_{a}^{b}
$$

which is equivalent to Eq. (4.42). Using the Hodge duality constraint (3.20b), the remaining spinor conditions $(4.56 a, b)$ contracted with $\gamma^{\alpha}$ and $\gamma^{n}$, respectively, become

$$
\begin{aligned}
& \left.\bar{\Omega}\lrcorner G_{(3)}=0, \quad(J\lrcorner G_{(3)}\right)^{(1,0)}=0, \quad\left(G_{(1)}\right)^{(1,0)}=0, \\
& \left.\bar{\Omega}\lrcorner \bar{G}_{(3)}=0, \quad(-i J\lrcorner \bar{G}_{(3)}+Z^{-1 / 2} \bar{G}_{(1)}\right)^{(1,0)}=0,
\end{aligned}
$$

where we have made use of the identity (4.59) $)$. Eq. (4.42a implies the Hodge duality condition (3.208). The constraints on $G_{(3)}$ are equivalent to demanding that $\widetilde{G}_{(3)}$ be primitive and of type $(2,1)+(1,2)$, where $\widetilde{G}_{(3)}$ is given by Eq. (4.42d).

Using this result, the original noncontracted constraints 4.56) eliminate the $(1,2)$ primitive piece via identity (4.59a). Thus, our final condition on $G_{(3)}$ for supersymmetry becomes Eq. (4.42b). This implies, among other things, that $G_{(3)}$ is imaginary-selfdual (ISD), which in turn reproduces the remaining Hodge duality condition (3.20d).

To summarize, the complete set of conditions for solutions to the equations of motion with $\mathcal{N} \geq 1$ supersymmetry consists of the constancy conditions (4.41), the constraints (3.20a) and (4.16a) relating the dilaton and $F_{(3)}^{0}$ to the warp factor, vanishing five-form flux (3.51), and finally the flux constraints (4.42a,b).

26 Similar identities have appeared in numerous places. See, for example ref. [71]. 


\section{Relation to T-Dual O3 Orientifolds}

The $\mathrm{O} 5$ orientifolds just discussed are T-dual to $\mathrm{O} 3$ orientifolds on $T^{2} \times K 3$ for $\mathcal{B}=K 3$ and on $T^{6}$ for $\mathcal{B}=T^{4}$. For the rest of the paper, we will restrict to the case that $\mathcal{B}=T^{4}$.

\subsection{Review of O3 Orientifolds with Internal $T^{6}$}

In this subsection and the next, symbols with (without) a prime denote quantities associated with the O3 (O5) orientifold. The orientifold projection for O3 planes has already been discussed in connection with the decomposition of the 10D supersymmetry parameters $\epsilon_{L, R}^{\prime}$ in Sec. 4.1. The even internal fluxes preserved by the orientifold projection are $F_{(3)}^{\prime}$ and $H_{(3)}^{\prime}$. Except in the case that O3 and D3 charges cancel locally, the equations of motion also require odd $F_{(5)}^{\prime}$ flux. For compact internal manifold $X_{6}^{\prime}$, the most general $\mathcal{N}^{\prime} \geq 1$ O3 orientifold background compatible with 4D Poincaré invariance is [6]

$$
\begin{aligned}
d s_{\text {string }}^{2} & =Z^{\prime-1 / 2} \eta_{\mu \nu} d x^{\mu} d x^{\nu}+Z^{1 / 2} d s_{6}^{2}, \\
e^{\phi^{\prime}} & =g_{s}^{\prime}=\mathrm{const}, \\
-\frac{1}{g_{s}^{\prime}} \nabla_{X_{6}^{\prime}}^{2} Z^{\prime} & =(2 \pi)^{4} \alpha^{\prime 2} \sum_{i} Q_{i}^{\prime}\left(\frac{\delta^{6}\left(x-x_{i}\right)}{\sqrt{g_{6}^{\prime}}}-\frac{1}{V_{6}^{\prime}}\right), \\
\widetilde{F}_{(5)}^{\prime} & =\left(1+*_{6}\right) d\left(g_{s}^{\prime-1} Z^{\prime-1} d x^{0} \wedge d x^{1} \wedge d x^{2} \wedge d x^{3}\right),
\end{aligned}
$$

where $X_{6}^{\prime}$ is a Calabi-Yau threefold, $K 3 \times T^{2}$, or $T^{6}$, and $V_{6}^{\prime}$ is the volume of $X_{6}^{\prime}$.

The Gauss's law constraint is

$$
N_{\text {flux }}^{\prime}+\sum_{i} Q_{i}^{\prime}=0, \quad \text { where } \quad N_{\text {flux }}^{\prime}=\frac{1}{(2 \pi)^{4} \alpha^{\prime 2}} \int_{X_{6}^{\prime}} F_{(3)}^{\prime} \wedge H_{(3)}^{\prime}
$$

Here, $\left\{\left(Q_{i}^{\prime}, x_{i}\right)\right\}$ is the set of charges and positions of local O3 and D3 sources, with $Q_{i}^{\prime}$ equal to 1 for a D3 brane. We assume that all O3 planes are standard O3- planes in the terminology of Ref. [59], and work on the covering space of the orientifold, so that there are $M$ D3 branes and $M \mathbb{Z}_{2}$ image branes. The orientifold operation is $\Omega(-1)^{F_{l}} \mathcal{I}_{6}$, where $I_{6}$ inverts $X_{6}^{\prime}$. For $X_{6}^{\prime}=T^{6}$, the $\mathrm{O} 3$ planes are located at the $2^{6}$ fixed points on the base where each $x^{a}$ is equal to 0 or $1 / 2$, and each O3 plane has charge $Q_{i}^{\prime}=-1 / 2$.

In the absence of three-form flux, the theory preserves $4 \mathrm{D} \mathcal{N}^{\prime}=4$ supersymmetry. The 4D moduli are the zero-modes on $X_{6}^{\prime}$ of

$$
\tau_{\mathrm{dil}}^{\prime}=C_{(0)}^{\prime}+i / g_{s}^{\prime}, \quad g_{6 a b}^{\prime}, \quad C_{(2) a b}^{\prime}, \quad \text { and } \quad C_{(4) a b c d}^{\prime}
$$


together with the D3 worldvolume scalars $\Phi_{I}, I=1, \ldots, M$. The massless $4 \mathrm{D}$ gauge bosons are the zero-modes of

$$
B_{(2) \mu a}^{\prime} \quad \text { and } \quad C_{(2) \mu a}^{\prime}
$$

together with the D3 worldvolume gauge bosons $A_{I \mu}$.

In the presence of three-form flux, the supersymmetry and massless field content is reduced [9]. For the case that $X_{6}^{\prime}=T^{6}$, this model has been analyzed in great detail [11, 60]. The possible choices of NS and RR three-form flux are $H_{(3)}^{\prime}, F_{(3)}^{\prime} \in(2 \pi)^{2} \alpha^{\prime} H^{3}\left(T^{6}, 2 \mathbb{Z}\right)$. That is,

$$
\begin{aligned}
H_{(3)}^{\prime} & =(2 \pi)^{2} \alpha^{\prime} m_{[a b c]} d x^{a} \wedge d x^{b} \wedge d x^{c}, \quad m_{[a b c]} \in 2 \mathbb{Z}, \\
F_{(3)}^{\prime} & =(2 \pi)^{2} \alpha^{\prime} n_{[a b c]} d x^{a} \wedge d x^{b} \wedge d x^{c}, \quad n_{[a b c]} \in 2 \mathbb{Z}, \quad a, b, c=1, \ldots, 6 .
\end{aligned}
$$

Without imposing any supersymmetry conditions, the equations of motion alone imply the ISD condition

$$
*_{6} G_{(3)}^{\prime}=i G_{(3)}^{\prime}
$$

where

$$
G_{(3)}^{\prime}=F_{(3)}^{\prime}-\tau_{\mathrm{dil}}{ }^{\prime} H_{(3)}^{\prime} .
$$

Since $H_{(3)}^{\prime}$ and $F_{(3)}^{\prime}$ are discrete, this is a constraint on $\tau_{\text {dil }}{ }^{\prime}$ and metric moduli.

The condition for $\mathcal{N}^{\prime} \geq 14 \mathrm{D}$ (Poincaré) supersymmetry further refines this to

$$
G_{(3)}^{\prime} \quad(2,1) \text { and primitive. }
$$

The complex structure on $T^{6}$ can be parametrized by a complex $3 \times 3$ period matrix ${\tau^{\prime i}}_{j}$,

$$
z^{i}=x^{i}+\tau^{\prime i}{ }_{j} y^{j},
$$

where $z^{i} \cong z^{i}+1 \cong z^{i}+\tau^{\prime i}{ }_{j}$, for $i, j=1,2,3$. The holomorphic three-form is

$$
\Omega^{\prime} \propto d z^{1} \wedge d z^{2} \wedge d z^{3}
$$

which depends on the complex structure moduli up to cubic order in the period matrix. The Kähler form is

$$
J^{\prime}=i g_{i \bar{\jmath}}^{\prime} d z^{i} \wedge d \bar{z}^{j} .
$$


The $(2,1)$ supersymmetry condition is easily implemented by varying the superpotential of Gukov, Vafa, and Witten [57]

$$
W_{\mathrm{GVW}}=\int_{X_{6}^{\prime}} G_{(3)}^{\prime} \wedge \Omega^{\prime},
$$

with respect to $\tau_{\text {dil }}{ }^{\prime}$ and all complex structure moduli $\tau^{\prime i}{ }_{j}$, and in addition imposing the condition $W_{\mathrm{GVW}}=0$. (The latter follows from $D_{\rho} W_{\mathrm{GVW}}=0$, where $\rho$ is the complexified overall volume modulus [9,11.) For generic supersymmetric vacua, this fixes $\tau_{\text {dil }}$ and all of the complex structure moduli, but for nongeneric flux, some of these moduli are left unfixed. The primitivity condition then becomes a linear constraint on the $g_{i \bar{\jmath}}^{\prime}$, with coefficients determined by the flux and axion-dilaton. One subtlety in this procedure for $X_{6}^{\prime}=T^{6}$ is that not all of the $(18$ real $) \tau^{\prime i}{ }_{j}$ and $(9$ real $) g_{i \bar{\jmath}}^{\prime}$ correspond to the $(21$ real $)$ physical metric moduli. See App. E for further discussion. Due to the subtleties discussed in App. E concerning $W_{\mathrm{GVW}}$ in theories with extended supersymmetry, we have chosen in this paper to avoid any reference to a superpotential for the $\mathrm{O} 5$ orientifold, and instead to state the supersymmetry conditions directly in terms of conditions on the fields.

In the $\mathrm{O} 3$ orientifold, the decomposition of RR potentials into a background part $C_{(p)}^{\text {bgg }}$ and deformation $c_{(p)}^{\prime}$,

$$
C_{(p)}^{\prime}=C_{(p)}^{\prime \mathrm{bg}}+c_{(p)}^{\prime},
$$

is cleaner than it was for the $\mathrm{O} 5$ orientifold. First, set the gauge fields to zero and focus on deformations corresponding to moduli. Then,

$$
C_{(0)}^{\prime}=c_{(0)}^{\prime}, \quad C_{(2)}^{\prime}=C_{(2)}^{\prime \mathrm{bg}},
$$

and it is only in $C_{(4)}^{\prime}$ that both contributions appear:

$$
C_{(4)}^{\prime}=C_{(4)}^{\prime \mathrm{bg}}+c_{(4)}^{\prime}
$$

Here, $F_{(3)}^{\prime}, H_{(3)}^{\prime}$, and $\widetilde{F}_{(5)}^{\prime}$, as given by Eqs. (5.4) and (5.1d), satisfy

$$
\begin{aligned}
& F_{(3)}^{\prime}=d C_{(2)}^{\prime \mathrm{bg}} \\
& \widetilde{F}_{(5)}^{\prime}=d C_{(4)}^{\prime \mathrm{bg}}-C_{(2)}^{\mathrm{bg}} \wedge H_{(3)}^{\prime} .
\end{aligned}
$$

In addition to the deformations just discussed, there are other deformations of the supergravity background which when promoted to 4D fields become gauge bosons. In the closed string sector, these deformations are the zero modes on $X_{6}^{\prime}$ of $B_{(2) d \mu}^{\prime}$ and $C_{(2) d \mu}^{\prime}$, 
and will be denoted by the lowercase symbols $b_{(2) d \mu}^{\prime}$ and $c_{(2) d \mu}^{\prime}$, respectively. When the couplings to gauge bosons are included and all deformations are promoted to 4D fields, the kinetic term for $c_{(4) a b c d}^{\prime}$ is the square of 60]

$$
\partial_{\mu} c_{(4) a b c f}^{\prime}+4 F_{(3)[a b c \mid}^{\prime} b_{(2) \mid f] \mu}^{\prime}-4 H_{(3)[a b c \mid}^{\prime} c_{(2) \mid f] \mu}^{\prime}
$$

If we define complex gauge bosons

$$
d_{a \mu}^{\prime}=c_{(2) a \mu}^{\prime}-\tau_{\mathrm{dil}}^{\prime} b_{(2) a \mu}^{\prime}, \quad \text { and } \quad \bar{d}_{a \mu}^{\prime}=c_{(2) a \mu}^{\prime}-\bar{\tau}_{\mathrm{dil}}^{\prime} b_{(2) a \mu}^{\prime}
$$

then Eq. (5.15) can also be written as

$$
\partial_{\mu} c_{(4) a b c f}^{\prime}-\frac{1}{\operatorname{Im} \tau_{\mathrm{dil}^{\prime}}} \operatorname{Im}\left(4 \bar{G}_{(3)[a b c \mid}^{\prime} d_{\mid f] \mu}^{\prime}\right) .
$$

\subsection{T-Duality Map}

In this subsection, as in the previous one, symbols with (without) a prime denote quantities associated with the $\mathrm{O} 3$ (O5) orientifold. Let $X_{6}^{\prime}=T^{6}$ and $\mathcal{B}=T^{4}$. Then, the metric on $X_{6}^{\prime}$ can be written as a trivial $T^{2}$ fibration over $\mathcal{B}$ :

$$
d s_{6}^{\prime 2}=d s_{\mathcal{B}}^{2}+g_{\alpha \beta}^{\left(T_{\text {fib }}^{2}\right) \prime} \eta^{\prime a} \eta^{\prime \beta}, \quad \eta^{\prime \alpha}=d x^{\alpha}+a^{\prime \alpha}
$$

where trivial means that $\mathcal{F}^{\prime \alpha}=d a^{\prime \alpha}=0$. The use of a lowercase $a^{\prime \alpha}$ indicates that the $a^{\prime \alpha}{ }_{m n}$ are moduli rather than a quantized background. Until the very end of this subsection, we set all gauge fields to zero.

For the O5 orientifold, we write NS $B$-field as $B_{(2)}=B_{(2)}^{\mathrm{bg}}+b_{(2)}$, where, from Sec. 3.9,

$$
\begin{aligned}
B_{(2)}^{\mathrm{bg}} & =B_{(2)}^{\mathrm{bg} 0}=\frac{1}{2} B_{(2) m n} d x^{m} \wedge d x^{n} \\
b_{(2)} & =b_{(2)}^{1}=\eta^{\alpha} \wedge b_{(2) \alpha}, \quad \text { with } \quad b_{(2) \alpha}=b_{(2) \alpha m} d x^{m} .
\end{aligned}
$$

The NS $B$-field for the O3 orientifold is given by $B_{(2)}^{\prime}=B_{(2)}^{\prime \mathrm{bg}}$, where $B_{(2)}^{\prime \mathrm{bg}}=B_{(2)}^{\prime \mathrm{bg} 0}+B_{(2)}^{\prime \mathrm{bg} 1}$, and

$$
\begin{aligned}
& B_{(2)}^{\text {bg0 }}=\frac{1}{2} B_{(2) m n}^{\prime \mathrm{bg}} d x^{m} \wedge d x^{n}, \\
& B_{(2)}^{\prime \mathrm{bg} 1}=\eta^{\prime \alpha} \wedge B_{(2) \alpha}^{\text {bg }}, \quad \text { with } \quad B_{(2) \alpha}^{\text {/bg }}=B_{(2) \alpha m}^{\text {/bg }} d x^{m} .
\end{aligned}
$$

T-duality is an exact duality between string vacua that relates conformal field theories order by order in string perturbation theory. At the level of the conformal field theories, it is has the simple interpretation as the sign reversal $X_{R}^{\alpha}(\bar{z}) \rightarrow-X_{R}^{\alpha}(\bar{z})$ of the right-moving worldsheet scalars in the duality directions. However, in the low energy supergravity 
description, it involves an intermediate operation known as smearing. In the O3 orientifold, the locations of the O3 planes and D3 branes spontaneously break the translation isometries in each of the internal $T^{6}$ directions. The breaking is spontaneous in the sense that, on the $\mathbb{Z}_{2}$ covering space, the entire O3/D3 system can be translated by an arbitrary amount along any of the $T^{6}$ circles, to produce an inequivalent vacuum with identical relative O3/D3 positions and identical physics. The space of vacua respects the isometry, but a particular vacuum does not. Smearing is the operation of averaging a supergravity background over all vacua related by such translations in order to restore an isometry. To perform the T-duality between the O3 and O5 supergravity backgrounds, we first smear in the 4 and 5 directions and then perform the supergravity analog of the conformal field theory T-duality in these directions. The information about D3 brane positions lost in the smearing is recovered in the Wilson lines of the of D5 worldvolume gauge fields in the 4 and 5 directions.

The effect of the smearing is that the warp factors $Z$ and $Z^{\prime}$ are related via

$$
Z=Z_{\mathrm{smeared}}^{\prime}=\frac{1}{V_{\mathrm{fib}}^{\prime}} \int_{T_{\mathrm{fib}}^{2},} \operatorname{Vol}_{\mathrm{fib}}^{\prime} Z^{\prime},
$$

where

$$
\mathrm{Vol}_{\mathrm{fib}}^{\prime}=V_{\mathrm{fib}}^{\prime} \eta^{\prime 4} \wedge \eta^{\prime 5}, \quad \text { and } \quad V_{\mathrm{fib}}^{\prime}=\left(g^{\left(T_{\mathrm{fib}}^{2}\right) \prime}\right)^{1 / 2}=(2 \pi)^{4} \alpha^{\prime 2} / V_{\mathrm{fib}},
$$

which is easily seen to relate solutions of the $6 \mathrm{D}$ Poisson equation (5.1d) to solutions of the 4D Poisson equation (3.29).

The T-duality action on the dilaton is

$$
e^{\phi}(2 \pi)^{2} \alpha^{\prime} /\left(Z^{-1} V_{\mathrm{fib}}\right)=e^{\phi^{\prime}}
$$

or equivalently

$$
g_{s}(2 \pi)^{2} \alpha^{\prime} / V_{\mathrm{fib}}=g_{s}^{\prime}
$$

The T-duality action on the NS $B$-field and metric interchanges the geometrical $S^{1}$ fibration of connection $a^{\prime \alpha}$ with the formal $S^{1}$ fibrations of connection $B_{(2) \alpha}^{\prime b g} .27$ The relations are 38

$$
\begin{aligned}
b_{(2) \alpha} & =-a^{\prime \alpha}(2 \pi)^{2} \alpha^{\prime}, \\
\mathcal{A}^{\alpha} & =-B_{(2) \alpha}^{\prime \mathrm{bg}} /\left((2 \pi)^{2} \alpha^{\prime}\right), \\
B_{(2)}^{\mathrm{bg}} & =B_{(2)}^{\prime \mathrm{bg} 0}+a^{\prime \alpha} \wedge B_{(2) \alpha}^{\prime \mathrm{bg}},
\end{aligned}
$$

27 This point has been emphasized recently, first in Ref. [72] (and subsequent work [73]) and then in Ref. [38]. 
together with

$$
\begin{aligned}
& g_{\alpha \beta}^{\left(T_{\mathrm{fib}}^{2}\right)}=g^{\left(T_{\mathrm{fib}}^{2}\right) / \alpha \beta}\left((2 \pi)^{2} \alpha^{\prime}\right)^{2}, \\
& g_{\mathcal{B} m n}=g_{\mathcal{B} m n}^{\prime} .
\end{aligned}
$$

Note that the T-duality map does not leave the purely base component $B_{(2)}^{0}$ invariant.28 Nevertheless, the correction term in Eq. (5.25d) has a straightforward interpretation. Let underscored indices $\underline{\mu}, \underline{\alpha}, \underline{m}$ denote components in the $d x^{\mu}, d x^{\alpha}, d x^{m}$ basis as opposed to the fibration-adapted bases involving $\eta^{\alpha}$ or $\eta^{\alpha}$. Then Eq. (5.257) is equivalent to

$$
B_{(2) m n}^{\mathrm{bg}}=B_{(2) \underline{m n}}^{\mathrm{bg}} .
$$

This illustrates an important rule. Many (but not all) of the T-duality relations connecting $\mathrm{O} 3$ and $\mathrm{O} 5$ orientifolds take the simplest form when expressed in terms of the $d x^{\alpha}, \eta^{\alpha}, d x^{m}$ basis for the O5 orientifold and the $d x^{\mu}, d x^{\alpha}, d x^{m}$ basis for the O3 orientifold. This is perhaps to be expected, since in the O3 orientifold there is nothing special about the $\alpha=4,5$ directions - any other pair of directions could have been used to define the fiber of a flat $T^{2}$ fibration. The three-form fluxes of the $\mathrm{O} 3$ orientifold are of the moduli-independent form (5.4). Thus, $H_{(3) \underline{\alpha m n}}^{\prime}$ and $H_{(3) \underline{m n r}}^{\prime}$ are quantized and moduli-independent, whereas $H_{(3) m n r}^{\prime}$ involves a combination of quantized fluxes and the metric moduli $a^{\prime \alpha}{ }_{m n}$. From the T-duality map (5.25), the relation between quantized NS sector fluxes is

$$
\mathcal{F}_{m n}^{\alpha}=H_{(3) \underline{\alpha m n}}^{\prime} /\left((2 \pi)^{2} \alpha^{\prime}\right) \quad \text { and } \quad H_{(3) m n r}^{\mathrm{bg}}=H_{(3) \underline{m n r}}^{\prime} .
$$

The relation between NS sector moduli has already been given explicitly in Eqs. (5.25a,d,e).

The rule described in the previous paragraph is particularly applicable to the T-duality action on RR fluxes and moduli. The T-duality relations between RR fluxes are [74, 64, 75,

$$
\begin{aligned}
\widetilde{F}_{(n)}^{0} & =\frac{1}{2} \epsilon^{\alpha \beta} \widetilde{F}_{(n+2) \alpha \beta}^{2} /\left((2 \pi)^{2} \alpha^{\prime}\right), \\
\widetilde{F}_{(n) \alpha}^{1} & =-\epsilon^{\alpha \beta} \widetilde{F}_{(n) \beta}^{1}, \\
\widetilde{F}_{(n) \alpha \beta}^{2} & =-\epsilon^{\alpha \beta} \widetilde{F}_{(n-2)}^{\prime 0}(2 \pi)^{2} \alpha^{\prime} .
\end{aligned}
$$

Here $\epsilon^{45}=-\epsilon^{54}=1$, and we have assumed that the ordering of the T-dualities in going from the $\mathrm{O} 3$ theory to the $\mathrm{O} 5$ theory is that we first T-dualize in the 4-direction and then in the 5-direction.

28 I am grateful to A. Tomasiello for correspondence regarding this point. 
In Eq. (5.28), both sides involve a combination of moduli-dependent and quantized contributions. It is desirable to disentangle the two types of contributions. When the RHS is expanded in terms of $a^{\prime \alpha}, c_{(0)}^{\prime}$, and underscored flux components, the relations become

$$
\begin{aligned}
& F_{(1) m}=\left(F_{(3)}^{\prime}-c_{(0)}^{\prime} H_{(3)}^{\prime}\right)_{\underline{45 m}} /(2 \pi)^{2} \alpha^{\prime}, \\
& F_{(3) \beta m n}=-\epsilon^{\beta \alpha}\left(F_{(3)}^{\prime}-c_{(0)}^{\prime} H_{(3)}^{\prime}\right)_{\underline{\alpha m n}}-2 a_{[\underline{[m} \mid}^{\prime \alpha}\left(F_{(3)}^{\prime}-c_{(0)}^{\prime} H_{(3)}^{\prime}\right)_{\underline{45} \underline{n}]}, \\
& \widetilde{F}_{(5) 45 m n r}=-\left(\left(F_{(3)}^{\prime}-c_{(0)}^{\prime} H_{(3)}^{\prime}\right)_{\underline{m n r}}-3 a^{\prime \alpha}{ }_{[\underline{m} \mid}\left(F_{(3)}^{\prime}-c_{(0)}^{\prime} H_{(3)}^{\prime}\right)_{\underline{\alpha} \mid \underline{n r}]}\right. \\
&\left.\quad+6 a_{[m \mid}^{\prime 4} a_{|n|}^{\prime 5}\left(F_{(3)}^{\prime}-c_{(0)}^{\prime} H_{(3)}^{\prime}\right)_{\underline{45} \mid \underline{r}]}\right)(2 \pi)^{2} \alpha^{\prime} .
\end{aligned}
$$

In addition, the map of odd fluxes is

$$
\widetilde{F}_{(3) m n r}=\left(\widetilde{F}_{(5) \text { smeared }}^{\prime}\right)_{45 m n r} /(2 \pi)^{2} \alpha^{\prime}
$$

Here, the five-form flux in the smeared supergravity backround is

$$
\widetilde{F}_{(5) \text { smeared }}^{\prime}=\frac{1}{g_{s}^{\prime}} \mathrm{Vol}_{\text {fib }}^{\prime} *_{\mathcal{B}} d Z_{\text {smeared }}^{\prime}
$$

By Eqs. (5.21) and (5.24), Eq. (5.29d) agrees with Eq. (3.200). In the remaining relations $(5.29 a, b, 6)$, we can drop the components $H_{(3)}^{\prime} \underline{45 m}$, which necessarily vanish for the Tduality map to exist (cf. Footnotes 7 and 12). Upon setting these terms to zero and using the map (5.25a), Eqs. (5.29c,d $)$ can be identified, term for term, with Eqs. (3.43) and (3.47a,b). The result is that the T-duality map between quantized fluxes is

$$
\begin{aligned}
F_{(1) m} & =F_{(3) \underline{45 m}}^{\prime} /(2 \pi)^{2} \alpha^{\prime}, \\
F_{(3) 4 m n}^{\mathrm{bg}} & =-F_{(3) \underline{5 m n}}^{\prime} \quad \text { and } \quad F_{(3) 5 m n}^{\mathrm{bg}}=F_{(3) \underline{4 m n}}^{\prime}, \\
F_{(5) 45 m n r}^{\mathrm{bg}} & =-F_{(3) \underline{m n r}}^{\prime}(2 \pi)^{2} \alpha^{\prime},
\end{aligned}
$$

and the axionic partner of the dilaton maps as

$$
\widetilde{c}_{(2) 45}=-(2 \pi)^{2} \alpha^{\prime} c_{(0)}^{\prime}
$$

From $H_{(3) \underline{45 m}}^{\prime}=0$ together with Eq. (5.5), the flux components $F_{(3) \underline{m n r}}^{\prime}$ vanish. Therefore,

$$
F_{(5) 45 m n r}^{\mathrm{bg}}=0
$$


which is a result that we stated without proof in Sec. 3.9. Eqs. (5.31d) and (5.24) together give

$$
\tau_{\text {dil }}=\tau_{\text {dil }}{ }^{\prime}
$$

where $\tau_{\text {dil }}$ is defined in Eq. (4.29) and $\tau_{\text {dil }}{ }^{\prime}$ in Eq. (5.2). See App. F for a discussion of the T-duality map between RR potentials.

The T-duality map between supersymmetry parameters is 75

$$
\begin{aligned}
& \epsilon_{R}=\epsilon_{R}^{\prime}, \\
& \epsilon_{L}=-\left(g^{\left(T_{\mathrm{fib}}^{2}\right)}\right)^{-1 / 2} Z^{-1 / 2} \Gamma^{(10)} \Gamma_{[5}^{\prime} \Gamma^{(10)} \Gamma_{4]}^{\prime} \epsilon_{L}^{\prime}=i \Gamma_{\mathrm{fib}} \epsilon_{L}^{\prime},
\end{aligned}
$$

in agreement with the results of Sec. 4.1. Here, as explained in Ref. [75], given a vielbein $e^{\prime A}{ }_{M}$ such that $g_{M N}^{\prime}=\eta_{A B} e^{A}{ }_{M} e^{B}{ }_{N}$ in the O3 theory, one obtains two different vielbeine $\left(e_{L, R}\right)_{M}^{A}$ in the dual O5 theory, and correspondingly two different Dirac matrix representations $\left(\Gamma_{L, R}\right)_{M}=\left(e_{L, R}\right)^{A}{ }_{M} \Gamma_{A}$, for fixed choice of representation of $\Gamma_{A}$ satisfying the algebra $\left\{\Gamma_{A}, \Gamma_{B}\right\}=2 \eta_{A B}$. The two vielbeine are related by a local Lorentz transformation. In writing Eq. (5.34), an arbitrary choice has been made to define

$$
\Gamma_{M}=\left(\Gamma_{R}\right)_{M}=\left(e_{R}\right)^{A}{ }_{M} \Gamma_{A}
$$

and to re-express $\left(e_{L}\right)^{A}{ }_{M}$ in terms of $\left(e_{R}\right)^{A}{ }_{M}$ whereever it appears. The net effect of this is to supplement T-duality with a local Lorentz transformation in the left moving worldsheet sector, which leads to nontrivial T-duality map of $\epsilon_{L}$.

Finally, when the deformations of the supergravity background corresponding to 4D gauge bosons are reintroduced, the T-duality map between these deformations is

$$
\begin{aligned}
& v_{\mu}^{\alpha}=-b_{(2) \alpha \mu}^{\prime} /\left((2 \pi)^{2} \alpha^{\prime}\right), \quad b_{(2) m \mu}=b_{(2) m \mu}^{\prime}, \\
& c_{(2) \alpha \mu}=-e^{\alpha \beta} c_{(2) \beta \mu}^{\prime}, \quad \widetilde{c}_{(4) 45 m \mu}=-c_{(2) m \mu}^{\prime}(2 \pi)^{2} \alpha^{\prime} .
\end{aligned}
$$

\section{6. $\mathcal{N}=2$ Examples}

In this section, we present three $\mathcal{N}=2$ examples in succession. The first two examples contain $F_{(3)}$ flux only, while the third example also contains $F_{(1)}$ and $H_{(3)}$ flux. The three examples are T-dual to the same O3 background, given in Sec. 6.4, via three different choices of the cycles on which to T-dualize. 


\subsection{Example 1: $\mathcal{N}_{+}=2, \mathcal{N}_{-}=0$}

Consider the choice of fibration curvature and background flux

$$
\begin{aligned}
& \mathcal{F}^{4}=2 n\left(d x^{6} \wedge d x^{8}+d x^{7} \wedge d x^{9}\right), \quad \mathcal{F}^{5}=0, \\
& F_{(3)}^{\mathrm{bg} 1} /\left((2 \pi)^{2} \alpha^{\prime}\right)=-2 m \eta^{4} \wedge\left(d x^{6} \wedge d x^{8}+d x^{7} \wedge d x^{9}\right),
\end{aligned}
$$

with $F_{(1)}=H_{(3)}=0$. This choice gives a contribution to the Gauss's law constraint,

$$
N_{\text {flux }}=8 m n,
$$

so that Eq. (3.15) becomes

$$
2 M+8 m n=32 .
$$

As discussed in Sec. 3.2, $N_{\text {flux }}$ is nonnegative. So, $m$ and $n$ have the same sign, and the number of $\mathrm{D} 5$ branes is $2 M=0,8,16,24$, or 32 , depending on the choice of integers $m$ and $n$. We assume that $m, n \neq 0$, so that $2 M$ is strictly less than 32 .

The complex three-form flux is

$$
\frac{1}{(2 \pi)^{2} \alpha^{\prime}} G_{(3)}=2 m\left(\eta^{4}+(n / m) \tau_{\mathrm{dil}} \eta^{5}\right) \wedge\left(d x^{6} \wedge d x^{8}+d x^{7} \wedge d x^{9}\right) .
$$

If we define an almost complex structure via (cf. Eq. (4.24))

$$
\begin{aligned}
& \eta^{z^{1}}=\eta^{4}+\tau_{1} \eta^{5}, \\
& d z^{i}=d x^{2 i+2}+\tau^{i}{ }_{j} d x^{2 j+3}, \quad i, j=2,3,
\end{aligned}
$$

then, using the decomposition (4.21), the type $(2,1)$ condition on the flux is

$$
\tau_{1}=(n / m) \tau_{\text {dil }} \quad \text { and } \quad \operatorname{det}_{2 \times 2} \tau=-1 .
$$

Note that the first condition implies that only for large fiber complex structure $\operatorname{Im} \tau_{1} \gg 1$ can we simultaneously have $g_{s} \ll 1$ and $V_{\text {fib }} \gg \alpha^{\prime}$. Eq. (6.6) is equivalent to demanding that the two factors appearing in $G_{(3)}$ be of definite Hodge type:

$$
\begin{aligned}
\eta^{4}-(n / m) \tau_{\text {dil }} \eta^{5} & \text { of type }(1,0), \\
d x^{6} \wedge d x^{8}+d x^{7} \wedge d x^{9} & \text { of type }(1,1) .
\end{aligned}
$$

Thus, from Eq. (6.1d), the complex fibration curvature $\mathcal{F}^{z^{1}}=\mathcal{F}^{4}+\tau_{1} \mathcal{F}^{5}$ is also of type $(1,1)$. This provides a check of our formal result that the complex structure is integrable 
in the case of RR three-form flux only. The vanishing of the $(0,2)$ component of $\mathcal{F}^{z^{1}}$ is all that is required for the fibration to be holomorphic; that condition is satisfied.

Finally, the primitivity condition is

$$
J^{\mathcal{B}} \wedge\left(d x^{6} \wedge d x^{8}+d x^{7} \wedge d x^{9}\right)=0
$$

Not all of the $\tau^{i}{ }_{j}$ and $g_{\mathcal{B} i j}$ left unfixed by these constraints correspond to independent physical moduli. (This is the $T^{4}$ version of the ambiguity discussed in App. E.) We can fix the redundancy at the cost of breaking manifest $S U(2)$ covariance in the parametrization of the metric and complex structure on the base $T^{4}$. We write the $T^{4}$ metric as a flat $T_{\{67\}}^{2}$ fibration over $T_{\{89\}}^{2}$,

$$
d s_{\mathcal{B}}^{2}=\frac{V_{2}}{\left|\operatorname{Im} \tau_{2}\right|}\left|\widetilde{\eta}^{6}+\tau_{2} \widetilde{\eta}^{7}\right|^{2}+\frac{V_{3}}{\left|\operatorname{Im} \tau_{3}\right|}\left|d x^{8}+\tau_{3} d x^{9}\right|^{2},
$$

where

$$
\begin{aligned}
& \widetilde{\eta}^{6}=\widetilde{a}^{6}{ }_{8} d x^{8}+\widetilde{a}^{6}{ }_{9} d x^{9}, \\
& \widetilde{\eta}^{7}=\widetilde{a}^{7}{ }_{8} d x^{8}+\widetilde{a}^{7}{ }_{9} d x^{9},
\end{aligned}
$$

with $\widetilde{\mathcal{F}}^{m}=d \widetilde{a}^{m}=0, m=6,7$. We take the $(1,0)$-forms on $X_{6}$ to be

$$
\eta^{z^{1}}=\eta^{4}+\tau_{1} \eta^{5}, \quad \widetilde{\eta}^{z^{2}}=\widetilde{\eta}^{6}+\tau_{2} \widetilde{\eta}^{7}, \quad \text { and } \quad d z^{3}=d x^{8}+\tau_{3} d x^{9},
$$

instead of those given in Eq. (6.5). Then,

$$
\begin{aligned}
\Omega^{\mathcal{B}} & =\left(\frac{V_{2} V_{3}}{\left|\operatorname{Im} \tau_{2}\right|\left|\operatorname{Im} \tau_{3}\right|}\right)^{1 / 2}\left(\widetilde{\eta}^{6}+\tau_{2} \widetilde{\eta}^{7}\right) \wedge\left(d x^{8}+\tau_{3} d x^{9}\right), \\
J^{\mathcal{B}} & =\left(\operatorname{sign} \tau_{2}\right) V_{2} \widetilde{\eta}^{6} \wedge \widetilde{\eta}^{7}+\left(\operatorname{sign} \tau_{3}\right) V_{3} d x^{8} \wedge d x^{9} .
\end{aligned}
$$

The $(2,1)$ condition becomes

$$
\begin{aligned}
\tau_{1} & =(n / m) \tau_{\text {dil }}, \\
\tau_{2} \tau_{3} & =-1,
\end{aligned}
$$

and the primitivity condition becomes

$$
\widetilde{a}_{9}^{6}=\widetilde{a}_{8}^{7}
$$

The constraints on NS $B$-field moduli follow from Eqs. (3.52a, $]$ ). These equations become

$$
0=h_{(3)}=b_{(2) 4 m} \mathcal{F}^{4} \wedge d x^{m}
$$


and

$$
0=-b_{(2) 5 m} d x^{m} \wedge F_{(3) 4}^{\mathrm{bg}},
$$

respectively. The first equation eliminates $b_{(2)} 4 m$, and the second $b_{(2)} 5 m$. So, there are no NS $B$-field moduli.

The massless RR moduli are those that do not couple to the gauge fields. (Those that do are eaten via the supersymmetric Higgs mechanism). From the kinetic terms (3.54), they are

$$
\widetilde{c}_{(2) 89}, \widetilde{c}_{(2) 78}, \widetilde{c}_{(2) 69}, \widetilde{c}_{(2) 67}, \widetilde{c}_{(2) 68}-\widetilde{c}_{(2) 79}, \quad \text { and } \quad \widetilde{c}_{(2) \mu \nu} \leftrightarrow \widetilde{c}_{(6) 456789}
$$

Similarly, the massless gauge bosons are those that do not couple to RR scalars. From (3.54), they are the three linear combinations of $v^{4}{ }_{\mu}, v^{5}{ }_{\mu}, c_{(2)} 4 \mu$, and $c_{(2)} 5 \mu$ orthogonal to

$$
(2 \pi)^{2} \alpha^{\prime}(m / n) v_{\mu}^{4}+c_{(2)} 4 \mu,
$$

where orthogonality is defined by the metric appearing in the gauge kinetic terms. (See Sec. 6.4 for further discussion in the context of the dual O3 orientifold).

Finally, there are $6 M$ massless scalars $A_{I \alpha}, \Phi_{I}^{m}$ and $M$ massless gauge bosons $A_{I \mu}$ from the D5 branes.

In summary, the massless bosonic fields are one graviton, $3+M$ vectors, and $16+6 M$ moduli. The moduli consist of

$$
\begin{gathered}
V_{\text {fib }}, V_{2}, V_{3}, 2 \text { indep } \tau, 3 \text { indep } \widetilde{a}_{n}^{m}, \\
6 \widetilde{c}_{(2)} \text { scalars, and } 6 M \text { D5 scalars. }
\end{gathered}
$$

These fields combine to form one $4 \mathrm{D} \mathcal{N}=2$ gravity multiplet, $2+M$ vector multiplets, and $3+M$ hypermultiplets.

The amount of unbroken supersymmetry is

$$
\mathcal{N}_{+}=2, \quad \mathcal{N}_{-}=0,
$$

in the notation of Sec. 4.2. To verify this, first note that since $V_{\text {fib }}, g_{s}>0$, we have $\operatorname{Im} \tau_{\text {dil }}>0$. Then, from the moduli constraints, $\operatorname{Im} \tau_{1}>0$. So, $J^{\text {fib }}=+V_{\text {ol }}$ fib, and we conclude that $\chi=\chi_{+}$and $\mathcal{N}_{-}=0$. Next, observe that there is exactly one antiholomorphic involution of $X_{6}$ compatible with the constraints on complex structure moduli:

$$
\tau_{1}, \tau^{i}{ }_{j} \rightarrow \tau_{1}, \bar{\tau}^{i}{ }_{j}
$$

(or equivalently, $\tau_{1}, \tau_{2}, \tau_{3} \rightarrow \tau_{1}, \bar{\tau}_{2}, \bar{\tau}_{3}$ in the complex structure (6.11). Therefore, there are two independent $6 \mathrm{D}$ Killing spinors. The spinors are of the form

$$
\chi_{1+}=\zeta_{+}^{\mathrm{fib}} \otimes \zeta_{+}^{\mathcal{B}}, \quad \chi_{2+}=\zeta_{+}^{\mathrm{fib}} \otimes \zeta_{+}^{\mathcal{B}^{*}},
$$

for some $\zeta_{+}^{\mathrm{fib}}$ and $\zeta_{+}^{\mathcal{B}}$, and are related by complex conjugation of the base. 


\subsection{Example 2: $\mathcal{N}_{+}=\mathcal{N}_{-}=1$}

Choose fibration curvature and background flux

$$
\begin{aligned}
& \mathcal{F}^{4}=2 n d x^{6} \wedge d x^{8}, \quad \mathcal{F}^{5}=2 n d x^{7} \wedge d x^{8}, \\
& F_{(3)}^{\text {bg } 1} /\left((2 \pi)^{2} \alpha^{\prime}\right)=-2 m\left(\eta^{4} \wedge d x^{7}-\eta^{5} \wedge d x^{6}\right) \wedge d x^{9} .
\end{aligned}
$$

Again, $m, n$, and $M$ are constrained by Eq. (6.3). The complex three-form flux is

$$
\frac{1}{(2 \pi)^{2} \alpha^{\prime}} G_{(3)}=2 n \tau_{\text {dil }}\left(\eta^{4} \wedge d x^{7}-\eta^{5} \wedge d x^{6}\right) \wedge\left(d x^{8}+(m / n)\left(-1 / \tau_{\text {dil }}\right) d x^{9}\right) .
$$

Define the complex structure as in Eq. (6.5). Then, the type $(2,1)$ condition gives

$$
\begin{aligned}
\tau_{2 \times 2} & =\operatorname{diag}\left(\tau_{2}, \tau_{3}\right), \\
\tau_{1} & =\tau_{2}, \\
\tau_{3} & =(m / n)\left(-1 / \tau_{\text {dil }}\right) .
\end{aligned}
$$

So, the base factorizes into $T_{\left\{x^{6}, x^{7}\right\}}^{2} \times T_{\left\{x^{8}, x^{9}\right\}}^{2}$ with respect to complex structure. Primitivity implies that $g_{\mathcal{B} i \bar{\jmath}}$ factorizes in the same way. Therefore, the base metric takes the form

$$
d s_{\mathcal{B}}^{2}=\frac{V_{2}}{\left|\operatorname{Im} \tau_{2}\right|}\left|d z^{2}\right|^{2}+\frac{V_{3}}{\operatorname{Im} \tau_{3}}\left|d z^{3}\right|^{2} .
$$

Here, we have removed the absolute value bars from $\operatorname{Im} \tau_{3}$ since the nonnegativity of $\operatorname{Im} \tau_{\text {dil }}$ and $m n$ implies nonnegativity of $\operatorname{Im}\left(-1 / \tau_{3}\right)$ and $\operatorname{Im} \tau_{3}$.

From Eqs. $(\overline{3.52 a, b})$, the constraints on NS $B$-field moduli are

$$
\begin{aligned}
0 & =h_{(3)}=\left(b_{(2) 4 m} \mathcal{F}^{4}+b_{(2) 5 m} \mathcal{F}^{5}\right) \wedge d x^{m}, \\
& =-2 n\left(b_{(2) 4 m} d x^{6}+b_{(2) 5 m} d x^{7}\right) \wedge d x^{8} \wedge d x^{m}
\end{aligned}
$$

and

$$
\begin{aligned}
0 & =b_{(2) 4 m} d x^{m} \wedge F_{(3) 5}^{\mathrm{bg}}-b_{(2) 5 m} d x^{m} \wedge F_{(3) 4}^{\mathrm{bg}}, \\
& =-2 m d x^{m} \wedge\left(b_{(2) 4 m} d x^{6}+b_{(2) 5 m} d x^{7}\right) \wedge d x^{9} .
\end{aligned}
$$

These equations are equivalent to

$$
\begin{array}{ll}
b_{(2) 49}=b_{(2) 59}=0, & b_{(2) 56}=b_{(2) 47}, \\
b_{(2) 48}=b_{(2) 58}=0, & b_{(2) 56}=b_{(2) 47},
\end{array}
$$


respectively. So, there are three unconstrained NS $B$-field moduli,

$$
b_{(2) 46}, \quad b_{(2) 57}, \quad \text { and } \quad b_{(2) 56}+b_{(2) 47} .
$$

In the RR sector, the uneaten moduli that follow from the kinetic terms (3.54) are the uncharged scalars

$$
\widetilde{c}_{(2) 67}, \widetilde{c}_{(2) 89}, \widetilde{c}_{(4) 4689}, \widetilde{c}_{(4) 5789}, \widetilde{c}_{(4) 4789}+\widetilde{c}_{(4) 5689}, \quad \text { and } \quad \widetilde{c}_{(2) \mu \nu} \leftrightarrow \widetilde{c}_{(6) 456789}
$$

The massless gauge bosons are those that do not couple to RR scalars. From (3.54), they are the three linear combinations of $b_{(2) 8 \mu}, b_{(2) 9 \mu}, \widetilde{c}_{(4) 458 \mu}$, and $\widetilde{c}_{(4) 459 \mu}$ orthogonal to

$$
(2 \pi)^{2} \alpha^{\prime}(m / n) b_{(2) 8 \mu}-\widetilde{c}_{(4) 459 \mu},
$$

where orthogonality is defined by the metric appearing in the gauge kinetic terms. (See Sec. 6.4 for further discussion in the context of the dual O3 orientifold).

Finally, there are $6 M$ massless scalars $A_{I \alpha}, \Phi_{I}{ }^{m}$ and $M$ massless gauge bosons $A_{I \mu}$ from the D5 branes.

In summary, as in Ex. 1, the massless bosonic fields are one graviton, $3+M$ vectors, and $16+6 M$ moduli. However, the moduli now consist of

$$
\begin{gathered}
V_{\text {fib }}, V_{2}, V_{3}, 2 \text { indep } \tau, 3 \text { indep } b_{(2) \alpha m}, \\
3 \widetilde{c}_{(2)} \text { scalars, } 3 \widetilde{c}_{(4)} \text { scalars, and } 6 M \text { D5 scalars. }
\end{gathered}
$$

These fields combine to form one $4 \mathrm{D} \mathcal{N}=2$ gravity multiplet, $2+M$ vector multiplets, and $3+M$ hypermultiplets.

The amount of unbroken supersymmetry is

$$
\mathcal{N}_{+}=1, \quad \mathcal{N}_{-}=1,
$$

in the notation of Sec. 4.2. Due to the factorization $\mathcal{B}=T_{\left\{z^{2}\right\}}^{2} \times T_{\left\{z^{3}\right\}}^{2}$ and the earlier observation that $\operatorname{Im} \tau_{3}>0$, we can write

$$
\chi_{ \pm}=\zeta_{ \pm}^{\mathrm{fib}} \otimes \zeta_{ \pm}^{\mathcal{B}}, \quad \text { where } \quad \zeta_{ \pm}^{\mathcal{B}}=\zeta_{ \pm}^{(2)} \otimes \zeta^{(3)},
$$

for some $\zeta_{ \pm}^{\text {fib }}, \zeta_{ \pm}^{(2)}$ and $\zeta^{(3)}$. Here, $\zeta^{(i)}$ is a spinor on $T_{\left\{z^{i}\right\}}^{2}$, and the chirality of $\zeta^{(3)}$ is fixed by the condition $\operatorname{Im} \tau_{3}>0$. The two $6 \mathrm{D}$ Killing spinors $\chi_{+}$and $\chi_{-}$are related by complex conjugation of $T_{\text {fib }}^{2}$ and $T_{\left\{z^{2}\right\}}^{2}$,

$$
\tau_{1}, \tau_{2}, \tau_{3} \rightarrow \bar{\tau}_{1}, \bar{\tau}_{2}, \tau_{3}
$$

that is,

$$
\zeta_{+}^{\mathrm{fib}}=\zeta_{-}^{\mathrm{fib} *}, \quad \zeta_{+}^{(2)}=\zeta_{-}^{(2)^{*}}
$$




\subsection{Example 3: More General Flux}

Choose fibration curvature and background flux

$$
\begin{aligned}
\mathcal{F}^{5} & =-2 n d x^{6} \wedge d x^{9} \\
F_{(1)} & =2 m d x^{6} \\
F_{(3)}^{\mathrm{bg} 1} /\left((2 \pi)^{2} \alpha^{\prime}\right) & =-2 m \eta^{5} \wedge d x^{7} \wedge d x^{8} \\
H_{(3)}^{\mathrm{bg}} /\left((2 \pi)^{2} \alpha^{\prime}\right) & =2 n d x^{7} \wedge d x^{8} \wedge d x^{9}
\end{aligned}
$$

Again, $m, n$, and $M$ are constrained by Eq. 6.3). The flux $G_{(3)}$ is

$$
\frac{1}{(2 \pi)^{2} \alpha^{\prime}} G_{(3)}=-2 m \eta^{5} \wedge d x^{7} \wedge d x^{8}-2 n \tau_{\mathrm{dil}} d x^{4} \wedge d x^{6} \wedge d x^{9}+b_{(2)} \wedge 2 m d x^{6} .
$$

From the moduli constraints, it is possible to show that $b_{(2)}, J$, and the complex structure all decompose as $(49) \oplus(5678)$. We omit the proof here, since it is tedious and uninstructive; however, this factorization is dual to an analogous factorization for the $\mathrm{O} 3$ orientifold discussed in the next subsection. Due to the factorization, we have

$$
d s_{X_{6}}^{2}=d s_{5678}^{2}+d s_{49}^{2}
$$

where the most general metric in the 5678 directions can be written as

$$
d s_{5678}^{2}=\frac{V_{1}}{\left|\operatorname{Im} \tau_{1}\right|}\left|\eta^{5}+\tau_{1} Z^{1 / 2} \widetilde{\eta}^{8}\right|^{2}+Z \frac{V_{2}}{\left|\operatorname{Im} \tau_{2}\right|}\left|d x^{6}+\tau_{2} d x^{7}\right|^{2},
$$

with

$$
\widetilde{\eta}^{8}=d x^{8}+\widetilde{a}_{m}^{8} d x^{m}, \quad m=6,7,
$$

parametrizing a flat $S^{1}$ fibration, and the most general metric in the 49 directions can be written

$$
d s_{49}^{2}=\frac{V_{3}}{\left|\operatorname{Im} \tau_{3}\right|}\left|d x^{4}+\tau_{3} Z^{1 / 2} d x^{9}\right|^{2} .
$$

Here $\tau_{1}$ and $\tau_{3}$ are pure imaginary, but $\tau_{2}$ can have both real and imaginary parts.

In this parametrization, the $(3,0)$ form and Kähler form are

$$
\Omega=\left(\frac{V_{1} V_{2} V_{3}}{\left|\operatorname{Im} \tau_{1}\right|\left|\operatorname{Im} \tau_{2}\right|\left|\operatorname{Im} \tau_{3}\right|}\right)^{1 / 2}\left(\eta^{5}+\tau_{1} Z^{1 / 2} \widetilde{\eta}^{8}\right) \wedge Z^{1 / 2}\left(d x^{6}+\tau_{2} d x^{7}\right) \wedge\left(d x^{4}+\tau_{3} Z^{1 / 2} d x^{9}\right)
$$


and

$$
J=Z^{1 / 2} v_{1} \eta^{5} \wedge \widetilde{\eta}^{8}+Z v_{2} d x^{6} \wedge d x^{7}+Z^{1 / 2} v_{3} d x^{4} \wedge d x^{9}
$$

where the lowercase $v_{i}$ are signed volumes,

$$
v_{i}=V_{i} \operatorname{sign}\left(\operatorname{Im} \tau_{i}\right), \quad i=1,2,3 \quad(\text { no sum })
$$

By expressing $H_{(3)}$ in terms of $\eta^{5}, \widetilde{\eta}^{8}, d x^{6}, d x^{7}, d x^{4}, d x^{9}$, and using the factorization of $b_{(2)}$, we find that

$$
\begin{aligned}
& \left.\frac{1}{2}(Z J \wedge J)\right\lrcorner\left(\operatorname{Vol}_{\mathrm{fib}} \wedge H_{(3)}\right) /\left((2 \pi)^{2} \alpha^{\prime}\right) \\
& \quad=2 n \frac{v_{\mathrm{fib}}}{v_{1} v_{3}}\left(d x^{7}+\frac{b_{(2) 58}}{(2 \pi)^{2} \alpha^{\prime}} d x^{6}-Z^{-1 / 2} \frac{v_{1}}{v_{2}}\left(\frac{b_{(2) 57}}{(2 \pi)^{2} \alpha^{\prime}}-\frac{b_{(2) 58}}{(2 \pi)^{2} \alpha^{\prime}} \widetilde{a}_{7}^{8}+\widetilde{a}_{6}^{8}\right) \eta^{5}\right) .
\end{aligned}
$$

From Eq. (4.42d), the complex one-form flux is then

$$
\begin{aligned}
G_{(1)}= & -2 n i \frac{(2 \pi)^{2} \alpha^{\prime} v_{\mathrm{fib}}}{v_{1} v_{3} g_{s}}\left(d x^{7}+\frac{1}{(2 \pi)^{2} \alpha^{\prime}}\left(b_{(2) 58}+i \frac{m}{n} \frac{v_{1} v_{3} g_{s}}{v_{\mathrm{fib}}}\right) d x^{6}\right. \\
& \left.-Z^{-1 / 2} \frac{v_{1}}{v_{2}}\left(\frac{b_{(2) 57}}{(2 \pi)^{2} \alpha^{\prime}}-\frac{b_{(2) 58}}{(2 \pi)^{2} \alpha^{\prime}} \widetilde{a}^{8}{ }_{7}+\widetilde{a}_{6}^{8}\right) \eta^{5}\right) .
\end{aligned}
$$

The supersymmetry condition that $G_{(1)}$ be of type $(0,1)$ implies that

$$
\begin{aligned}
\frac{1}{\tau_{2}} & =\frac{1}{(2 \pi)^{2} \alpha^{\prime}}\left(b_{(2) 58}-i \frac{m}{n} \frac{v_{1} v_{3} g_{s}}{v_{\mathrm{fib}}}\right), \\
\widetilde{a}_{6}^{8} & =-\frac{1}{(2 \pi)^{2} \alpha^{\prime}}\left(b_{(2) 57}-b_{(2) 58} \widetilde{a}_{7}^{8}\right) .
\end{aligned}
$$

The second equation is equivalent to

$$
\widetilde{a}_{6}^{8}=-b_{(2) 57} /\left((2 \pi)^{2} \alpha^{\prime}\right)
$$

where the indices $\widetilde{6}, \widetilde{7}, \widetilde{8}$ refer to components in the $d x^{6}, d x^{7}, \widetilde{\eta}^{8}$ basis (cf. Eq. (6.37d)).

When these constraints are satisfied,

$$
G_{(1)}=-\frac{2 n i}{\bar{\tau}_{2}} \frac{(2 \pi)^{2} \alpha^{\prime} v_{\mathrm{fib}}}{v_{1} v_{3} g_{s}} d \bar{z}^{2}, \quad \text { where } \quad d z^{2}=d x^{6}+\tau_{2} d x^{7}
$$


This takes care of the condition on $G_{(1)}$. The remaining supersymmetry condition (4.42b) on $\widetilde{G}_{(3)}$ is most easily imposed by first demanding the weaker condition

$$
\left.Z^{1 / 2} J\right\lrcorner G_{(3)}=i G_{(1)}
$$

(cf. Eq. (4.62)). From the above expressions for $J$ and $G_{(3)}$, we obtain

$$
\left.Z^{1 / 2} J\right\lrcorner G_{(3)}=2 m \frac{(2 \pi)^{2} \alpha^{\prime}}{v_{1}}\left(d x^{7}+\left(\frac{v_{1}}{v_{3}}\left(\frac{n}{m} \tau_{\text {dil }}+\frac{b_{(2) 49}}{(2 \pi)^{2} \alpha^{\prime}}\right)+\frac{b_{(2) 58}}{(2 \pi)^{2} \alpha^{\prime}}\right) d x^{6}\right) .
$$

Identifying this with $i G_{(1)}$ gives the constraint

$$
(n / m) \tau_{\text {dil }}=\left(-b_{(2) 49}+i v_{3}\right) /\left((2 \pi)^{2} \alpha^{\prime}\right) .
$$

This constraint allows us to simplify Eq. (6.42a) to

$$
-1 / \tau_{2}=\left(-b_{(2)} 58+i v_{1}\right) /\left((2 \pi)^{2} \alpha^{\prime}\right)
$$

The tilded three-form flux is then

$$
\widetilde{G}_{(3)}=\left((2 \pi)^{2} \alpha^{\prime} m /\left(v_{1} \tau_{2}\right)\right)\left(v_{1} \eta^{5} \wedge \widetilde{\eta}^{8}-v_{3} d x^{4} \wedge d x^{9}\right) \wedge\left(d x^{6}+\tau_{2} d x^{7}\right) .
$$

The first factor is $(1,1)$ and primitive, and the second is $d z^{2}$. So, $\widetilde{G}_{(3)}$ is $(2,1)$ and primitive, and no further constraints arise from condition (4.42b).

From the kinetic terms (3.54), the massless RR scalars are the uncharged fields

$$
\widetilde{c}_{(2)} \widetilde{79}_{9}, \quad \widetilde{c}_{(2) 89}, \quad \widetilde{c}_{(4) 5789}+(2 \pi)^{2} \alpha^{\prime} \widetilde{c}_{(2)} \widetilde{69}_{9}, \quad \widetilde{c}_{(4) 5679}, \quad \widetilde{c}_{(4) 5689}, \quad \text { and } \quad \widetilde{c}_{(4) 4678}
$$

The massless gauge bosons are those that do not couple to RR scalars. From (3.54), they are the three linear combinations of $v^{4}{ }_{\mu}, b_{(2)}{ }_{9 \mu}, c_{(2)}{ }_{5 \mu}$, and $\widetilde{c}_{(4)} 459 \mu$ orthogonal to

$$
(m / n) b_{(2) 9 \mu}-c_{(2) 5 \mu},
$$

where orthogonality is defined by the metric appearing in the gauge kinetic terms. (See Sec. 6.4 for further discussion in the context of the dual O3 orientifold).

Finally, there are $6 M$ massless scalars $A_{I \alpha}, \Phi_{I}{ }^{m}$ and $M$ massless gauge bosons $A_{I \mu}$ from the D5 branes. 
In summary, as in the previous two examples, the massless bosonic fields are one graviton, $3+M$ vectors, and $16+6 M$ moduli. However, in this case the moduli can be taken to be the independent fields

$$
\begin{aligned}
& \tau_{1}, \tau_{2}, \tau_{3}, v_{2}, \tau_{\mathrm{dil}}, \widetilde{a}_{6}^{8}, \widetilde{a}^{8}, b_{(2) 5 \widetilde{5}}, \\
& 6 \widetilde{c}_{(p)} \text { scalars, and } 6 M \text { D5 scalars, }
\end{aligned}
$$

with $\tau_{1}$ and $\tau_{3}$ pure imaginary. These fields combine to form one $4 \mathrm{D} \mathcal{N}=2$ gravity multiplet, $2+M$ vector multiplets, and $3+M$ hypermultiplets.

The amount of unbroken supersymmetry is

$$
\mathcal{N}=2,
$$

due to the existence of an antiholomorphic involution on $\tau_{2}$ compatible with the moduli constraints,

$$
\tau_{2}, \rho_{1 \overline{1}} \rightarrow \bar{\tau}_{2},\left(\rho_{1 \overline{1}}\right)^{*}, \quad \text { where } \quad \rho_{1 \overline{1}}=\left(-b_{(2)} 58+i v_{1}\right) /\left((2 \pi)^{2} \alpha^{\prime}\right) .
$$

In contrast to the previous two examples, there is no decomposition of the $\mathcal{N}=2$ algebra into $\mathcal{N}_{+}$and $\mathcal{N}_{-}$algebras generated by $6 \mathrm{D}$ spinors of definite fiber chirality. For any $\mathcal{N}=1$ subalgebra of the $\mathcal{N}=2$, the corresponding $6 \mathrm{D}$ spinor $\hat{\chi}$ contains components of both positive and negative fiber chirality (cf. Sec. 4.4).

\section{Nonintegrability of the Almost Complex Structure}

In the example just discussed, the ACS selected by the supersymmetry conditions is nonintegrable. The simplest way to verify this is via the torsion classes $\mathcal{W}_{i}$ of Eq. (1.5). This is the one place in the paper in which we will find it useful to compute any of the $\mathcal{W}_{i}$. The criterion that we will use is [45, 32, 29]:

The ACS is integrable if and only if $\mathcal{W}_{1}=\mathcal{W}_{2}=0$

In the example, the ACS is such that the three (1,0)-forms of $X_{6}$ are the three factors appearing in $\Omega$ of Eq. (6.39a). Consequently, the three terms in $J$ of Eq. (6.397) are each of type $(1,1)$, and we have the following Hodge decomposition of $d J$ :

$$
\begin{aligned}
& d J_{(2,1)+(1,2)}=d Z \wedge\left(\frac{1}{2} Z^{-1 / 2} v_{1} \eta^{5} \wedge \widetilde{\eta}^{8}+v_{2} d x^{6} \wedge d x^{7}+\frac{1}{2} Z^{-1 / 2} v_{3} d x^{4} \wedge d x^{9}\right), \\
& d J_{(3,0)+(0,3)}=2 n Z^{1 / 2} v_{1} d x^{6} \wedge d x^{9} \wedge \widetilde{\eta}^{8}
\end{aligned}
$$


From the second equation, $\mathcal{W}_{1} \neq 0$. It is straightfoward to compute

$$
\mathcal{W}_{1}=-\frac{n \tau_{2}}{3 Z}\left(\frac{v_{1} /\left(v_{2} v_{3}\right)}{\operatorname{Im} \tau_{1} \operatorname{Im} \tau_{2} \operatorname{Im} \tau_{3}}\right)^{1 / 2} .
$$

Therefore, the ACS is nonintegrable.

Note, however, that there is no topological obstruction to definining an integrable complex structure. For example, the fibration (6.34b) is compatible with the complex structure

$$
w^{1}=x^{4}+i x^{5}, \quad w^{2}=x^{6}+i x^{9}, \quad \text { and } \quad w^{3}=x^{7}+i x^{8} .
$$

In this complex structure, $\mathcal{F}^{w^{1}}=-2 n d w^{2} \wedge d \bar{w}^{2}$, with no $(0,2)$ component, so that the fibration is indeed holomorphic. This can also be seen from the fact that $X_{6}$ is just a warped version of one of the complex nilmanifolds classified in Ref. [76]. On the other hand, the complex structure (6.54) is not the one selected by the supersymmetry conditions, and is incompatible with the physical metric (6.36) except at certain points in moduli space.

\subsection{Dual О3 Orientifold}

Consider the $\mathrm{O} 3$ orientifold with internal $T^{6}$ and flux 11]

$$
\begin{aligned}
& F_{(3)}^{\prime} /\left((2 \pi)^{2} \alpha^{\prime}\right)=2 m\left(d x^{4} \wedge d x^{6}+d x^{5} \wedge d x^{7}\right) \wedge d x^{9} \\
& H_{(3)}^{\prime} /\left((2 \pi)^{2} \alpha^{\prime}\right)=2 n\left(d x^{4} \wedge d x^{6}+d x^{5} \wedge d x^{7}\right) \wedge d x^{8}
\end{aligned}
$$

The Gauss's law constraint (5.1e) is Eq. (6.3). The complex flux $G_{(3)}^{\prime}$ is

$$
\frac{1}{(2 \pi)^{2} \alpha^{\prime}} G_{(3)}^{\prime}=-2 n \tau_{\mathrm{dil}}{ }^{\prime}\left(d x^{4} \wedge d x^{6}+d x^{5} \wedge d x^{7}\right) \wedge\left(d x^{8}-(m / n)\left(1 / \tau_{\mathrm{dil}}{ }^{\prime}\right) d x^{9}\right) .
$$

If we parametrize the complex structure as in Eq. (5.8), then the supersymmetry conditions imply a factorization $T^{6} \rightarrow T_{\{4567\}}^{4} \times T_{\{89\}}^{2}$ with respect to both complex and Kähler structure. The condition that $G_{(3)}^{\prime}$ be of type $(2,1)$ implies

$$
\tau^{\prime}=\tau_{T^{4}}^{\prime} \oplus \tau_{T^{2}}^{\prime}, \quad \text { with } \quad \operatorname{det} \tau_{T^{4}}^{\prime}=-1, \quad \tau_{\mathrm{dil}}{ }^{\prime} \tau_{T^{2}}^{\prime}=-m / n,
$$

where $\tau_{T^{4}}^{\prime}$ is a complex $2 \times 2$ matrix and $\tau_{T^{2}}^{\prime}$ is a complex number. The condition that $G_{(3)}^{\prime}$ be primitive then gives

$$
J^{\prime}=J_{T^{4}}^{\prime}+J_{T^{2}}^{\prime} .
$$


Working in terms of $\tau^{\prime i}{ }_{j}$ and $g_{i \bar{\jmath}}^{\prime}$ has been convenient thus far for deriving the $T^{6} \rightarrow$ $T^{4} \times T^{2}$ factorization, but is bad for describing moduli. As discussed in Sec. 5.1 and in App. E, the parametrization is redundent. For this reason, we will adopt a different parametrization shortly. First, however, note that the supersymmetry constraints on the $T^{4}$ factor can be phrased in a parametrization independent way as follows:

$$
d x^{4} \wedge d x^{6}+d x^{5} \wedge d x^{7} \quad(1,1) \text { and primitive on } T^{4}
$$

Starting from this condition, it is not hard to show that there is $\mathcal{N}=2$ supersymmetry. For example, at $g_{m n}^{\prime\left(T^{4}\right)}=\delta_{m n}$, there is an $S^{2}$ of complex structures such that the condition is satisfied. The corresponding Kähler forms and holomorphic $(2,0)$ forms are

$$
J_{T^{4}}^{\prime}=n^{A} J^{(A)}, \quad \Omega_{T^{4}}^{\prime}=n^{A} \Omega^{(A)}
$$

where $\mathbf{n}=\left(n^{1}, n^{2}, n^{3}\right)$ is a unit vector on $S^{2}$, and

$$
\begin{gathered}
J^{(1)}=d x^{4} \wedge d x^{5}+d x^{6} \wedge d x^{7}, \quad J^{(2)}=d x^{4} \wedge d x^{7}+d x^{5} \wedge d x^{6}, \quad J^{(3)}=d x^{4} \wedge d x^{6}-d x^{5} \wedge d x^{7} \\
\Omega^{(1)}=J^{(2)}+i J^{(3)}, \quad \Omega^{(2)}, \Omega^{(3)}=\text { cyc. perms. }
\end{gathered}
$$

The $S^{2}$ of complex structures defines a single hyperKähler structure, and corresponds to two $6 \mathrm{D}$ negative chirality Weyl spinors $\hat{\chi}_{1,2}^{\prime}$ via

$$
J^{\prime(A)}{ }_{a}^{b}=i \hat{\chi}^{\prime \dagger} \sigma^{(A)} \gamma_{a}^{b} \hat{\chi}, \quad \hat{\chi}^{\prime}=\left(\begin{array}{c}
\hat{\chi}_{1}^{\prime} \\
\hat{\chi}_{2}^{\prime}
\end{array}\right)
$$

Here, the primes indicates that we are discussing the $\mathrm{O} 3$ orientifold, and the hats indicate that $\chi^{\prime}$ is a normalized spinor, rescaled relative to the $\chi$ appearing in Eq. (4.12). One can also see the $\mathcal{N}=2$ supersymmetry from the fact that there exists exactly one antiholomorphic involution of $X_{6}^{\prime}$ that preserves the moduli constraints on $\tau^{\prime i}{ }_{j}$ and $\tau_{\text {dil }}{ }^{\prime}$,

$$
\tau_{T^{4}}^{\prime}, \tau_{T^{2}}^{\prime} \rightarrow \bar{\tau}_{T^{4}}^{\prime}, \tau_{T^{2}}^{\prime}
$$

This involution implies that for every Killing spinor such that $\hat{\chi}_{1}^{\prime}=\chi_{T^{4}}^{\prime} \otimes \chi_{T^{2}}^{\prime}$, there is an independent Killing spinor such that $\chi_{2}^{\prime}=\chi_{T^{4}}^{\prime}{ }^{*} \otimes \chi_{T^{2}}^{\prime}$. The condition $\operatorname{Im} \tau_{T^{2}}^{\prime}=$ $(m / n) \operatorname{Im}\left(-1 / \tau_{\text {dil }}{ }^{\prime}\right)>0$, fixes the chirality of $\chi_{T^{2}}^{\prime}$ to be positive. Since $\chi^{\prime}$ has negative chirality by definition, this means that $\chi_{T^{4}}^{\prime}$ also has negative chirality. On $T^{4}$, there are exactly two negative chirality spinors, complex conjugate to one another up to an overall 
phase that can be absorbed into the definition of the spinors. Therefore, once we know that this example preserves any supersymmetry, we know that it preserves $\mathcal{N}=2$.

A nonredundent parametrization of the metric moduli is obtained by writing the $T^{4}$ as a flat $T_{\{45\}}^{2}$ fibration over $T_{\{67\}}^{2}$ :

$$
\begin{aligned}
& d s_{T^{4}}^{2}=\frac{V_{1}^{\prime}}{\operatorname{Im} \tau_{1}^{\prime}}\left|\eta^{\prime 4}+\tau_{1}^{\prime} \eta^{\prime 5}\right|^{2}+\frac{V_{2}^{\prime}}{\operatorname{Im} \tau_{2}^{\prime}}\left|d x^{6}+\tau_{2}^{\prime} d x^{7}\right|^{2}, \\
& d s_{T^{2}}^{2}=\frac{V_{3}^{\prime}}{\operatorname{Im} \tau_{3}^{\prime}}\left|d x^{8}+\tau_{3}^{\prime} d x^{9}\right|^{2},
\end{aligned}
$$

where

$$
\begin{aligned}
& \eta^{4}=d x^{4}+a^{\prime 4}{ }_{6} d x^{6}+a^{\prime 4}{ }_{7} d x^{7}, \\
& \eta^{\prime 5}=d x^{5}+a^{\prime 5}{ }_{6} d x^{6}+a^{\prime 5}{ }_{7} d x^{7},
\end{aligned}
$$

with $a^{\prime m}{ }_{n}$ constant on $T^{6}$. That is, $a^{\prime m}$ defines a trivial fibration $\mathcal{F}^{\prime m}=d a^{\prime m}=0$ over the base $T_{\{6,7\}}^{2}$. The natural holomorphic one-forms associated with this parametrization are

$$
\eta^{\prime z^{1}}=\eta^{4}+\tau_{1}^{\prime} \eta^{\prime 5}, \quad d z^{2}=d x^{6}+\tau_{2}^{\prime} d x^{7}, \quad \text { and } \quad d z^{3}=d x^{8}+\tau_{3}^{\prime} d x^{9} .
$$

Therefore, the $(2,1)$ condition on $G_{(3)}$ becomes

$$
\begin{aligned}
\tau_{1}^{\prime} \tau_{2}^{\prime} & =-1, \\
\tau_{3}^{\prime} \tau_{\text {dil }}{ }^{\prime} & =-m / n,
\end{aligned}
$$

and the primitivity condition becomes

$$
a^{14}=a_{6}^{15} .
$$

From the kinetic terms (5.15), the massless $c_{(4) a b c d}$ scalars are the uncharged scalars

$$
c_{(4) 6789}^{\prime}, \quad c_{(4) 5689}^{\prime}, \quad c_{(4) 4789}^{\prime}, \quad c_{(4) 4589}^{\prime}, \quad c_{(4) 4689}^{\prime}-c_{(4) 5789}^{\prime}, \quad \text { and } \quad c_{(4) 4567}^{\prime} .
$$

The massless gauge bosons are those that do not couple to RR scalars. From (5.15), they are the three linear combinations of $b_{(2) 8 \mu}^{\prime}, b_{(2) 9 \mu}^{\prime}, c_{(2) 8 \mu}^{\prime}$, and $c_{(2) 9 \mu}^{\prime}$ orthogonal to

$$
(m / n) b_{(2) 8 \mu}^{\prime}+c_{(2) 9 \mu}^{\prime}
$$


Here, orthogonality is defined by the metric appearing in the gauge kinetic terms. In term of the complex gauge bosons (5.16), the metric on the 89 subspace is proportional to

$$
\frac{1}{2}\left(\begin{array}{ll}
0 & 1 \\
1 & 0
\end{array}\right) \bar{d}^{\prime} \quad \otimes \frac{1}{2}\left(\begin{array}{ll}
0 & 1 \\
1 & 0
\end{array}\right) \bar{z}^{3} .
$$

The massive linear combination (6.69) is proportional to $\operatorname{Re}\left(\left(1 / \bar{\tau}^{\prime}\right) d_{z^{3} \mu}^{\prime}\right)$.2. Therefore, the 3D space of massless gauge bosons is spanned by

$$
\operatorname{Im}\left(\left(1 / \bar{\tau}^{\prime}\right) d_{z^{3} \mu}^{\prime}\right), \quad \operatorname{Re}\left(d_{\bar{z}^{3} \mu}^{\prime}\right), \quad \text { and } \operatorname{Im}\left(d_{\bar{z}^{3} \mu}^{\prime}\right) .
$$

Finally, there are $6 M$ massless scalars $\Phi_{I}^{\prime m}$ and $M$ massless gauge bosons $A_{I \mu}^{\prime}$ from the D3 branes.

In summary, the massless bosonic fields are one graviton, $3+M$ vectors, and $16+M$ moduli. The moduli consist of

$$
\begin{gathered}
V_{1}^{\prime}, V_{2}^{\prime}, V_{3}^{\prime}, 2 \text { independent } \tau^{\prime}, 3 \text { independent } a_{n}^{\prime m}, \\
6 c_{(4)}^{\prime} \text { scalars, and } 6 M \text { D3 scalars. }
\end{gathered}
$$

From the supersymmetry-breaking mass spectra given in Ref. [17], this corresponds to $\mathcal{N}=4 \rightarrow \mathcal{N}=2$ with $m_{1}=m_{2}$ for the two massive gravitini. (This is a third way to see that the background preserves $\mathcal{N}=2$ supersymmetry). The moduli space is $\mathcal{M}=\mathcal{M}_{H} \times \mathcal{M}_{V}$, where in the approximation that the warp factor is set to unity,

$$
\begin{aligned}
\mathcal{M}_{H} & =\frac{S O(4,3+M)}{S O(4) \times S O(3+M)}, \\
\mathcal{M}_{V} & =\frac{S O(2,1+M)}{S O(2) \times S O(1+M)} \times \frac{S U(1,1)}{U(1)},
\end{aligned}
$$

up to discrete identifications. See App. G for a discussion of the metric on moduli space.

\subsection{T-Duality Map}

The T-duality map relating the O3 orientifold of Sec. 6.4 to the O5 orientifolds of Secs. 6.1-6.3 is exactly as described in Sec. 5.2, once we perform the following relabelings of coordinates in the $\mathrm{O} 3$ orientifold:

Sec. 6.1: $\quad\left(x^{4}, x^{5}, x^{6}, x^{7}, x^{8}, x^{9}\right)_{\text {Sec. } 6.4}=\left(x^{6}, x^{7}, x^{8}, x^{9}, x^{4}, x^{5}\right)_{\text {new }}$,

Sec. 6.2: no relabeling,

Sec. 6.3: $\quad\left(x^{4}, x^{5}, x^{6}, x^{7}, x^{8}, x^{9}\right)_{\text {Sec. } 6.4}=\left(x^{5}, x^{8}, x^{6}, x^{7},-x^{9}, x^{4}\right)_{\text {new }}$.

29 Note that from $d z^{3}=d x^{8}+\tau^{\prime} d x^{9}$, we have $d_{z^{3} \mu}^{\prime}=\left(d_{9 \mu}^{\prime}-\bar{\tau}^{\prime} d_{8 \mu}\right) /\left(\tau^{\prime}-\bar{\tau}^{\prime}\right)$. 
Note that in all three cases, the relabeling is a permutation of positive Jacobian. Therefore, the image of the ISD flux (6.55) is again ISD, rather than IASD. In terms of the $T^{6} \rightarrow$ $T^{4} \times T^{2}$ factorization of Sec. 6.4, the three relabelings correspond to T-dualizing along the $T^{2}$ factor, along a $T^{2}$ in the $T^{4}$ factor, and along $S^{1} \subset T^{4}$ times $S^{1} \subset T^{2}$, respectively.

It is straightforward to follow all moduli and gauge fields through the T-duality map, using the relations in Sec. 5.2. The constraints (6.66a,b) and (6.67) map to 6.13b,a) and (6.14) in Sec. 6.1, to $(6.23 a, b)$ and $b_{(2) 56}=b_{(2) 47}$ in Sec. 6.2, and to $(6.46 b, a)$ and $(6.42 b)$ in Sec. 6.3. The volumes $V_{1}^{\prime}, V_{2}^{\prime}, V_{3}^{\prime}$ are equal to $V_{2}, V_{3},(2 \pi)^{4} \alpha^{\prime 2} / V_{\text {fib }}$ in Sec. 6.1, to $(2 \pi)^{4} \alpha^{\prime 2} / V_{\text {fib }}, V_{2}, V_{3}$ in Sec. 6.2 , and to $(2 \pi)^{2} \alpha^{\prime}\left|\tau_{1}\right|, V_{2},(2 \pi)^{2} \alpha^{\prime}\left|\tau_{3}\right|$ in Sec. 6.3. The RR moduli (6.68) map to (6.16), (6.28), and (6.48). The massive gauge bosons (6.69) map to (6.17), 6.29), and (6.49).

\section{7. $\mathcal{N}=3$ Example: The Warped Iwasawa Manifold}

\subsection{Example 4: $\mathcal{N}+=1, \mathcal{N}_{-}=2$}

Consider the choice of fibration curvature and background flux

$$
\begin{gathered}
\mathcal{F}^{4}=2 h\left(d x^{6} \wedge d x^{8}-m d x^{6} \wedge d x^{9}-m d x^{7} \wedge d x^{8}+\left(m^{2}-n\right) d x^{7} \wedge d y^{9}\right), \\
\mathcal{F}^{5}=-2 h\left(d x^{6} \wedge d x^{9}+d x^{7} \wedge d x^{8}-m d x^{7} \wedge d x^{9}\right), \\
F_{(3)}^{\mathrm{bg} 1} /\left((2 \pi)^{2} \alpha^{\prime}\right)=2 f\left(\eta^{4} \wedge\left(d x^{6} \wedge d x^{8}-n d x^{7} \wedge d x^{9}\right)\right. \\
\left.\quad+\eta^{5} \wedge\left(-n d x^{6} \wedge d x^{9}-n d x^{7} \wedge d x^{8}+m n d x^{7} \wedge d x^{9}\right)\right),
\end{gathered}
$$

with $F_{(1)}=H_{(3)}=0$. Here, $f, h, n=1,2$ and $m=0,1$. This choice gives a contribution to the Gauss's law constraint,

$$
N_{\text {flux }}=4 f h\left(4 n-m^{2}\right)
$$

so that Eq. (3.15) becomes

$$
2 M+4 f h\left(4 n-m^{2}\right)=32 .
$$

The condition $0 \leq N_{\text {flux }} \leq 32$ puts restrictions on the allowed combinations of $f, h, m, n$. The discussion of the dual O3 theory in Sec. 7.2 shows why this is in some sense a natural class of backgrounds to consider. 
For the choice (7.1) to preserve $\mathcal{N}=3$ supersymmetry, we require that the complex flux (4.28d) be $(2,1)$ and primitive with respect to three independent complex structures. This is equivalent to demanding that $G_{(3)}$ be of type $(0,3)$ with respect a single complex structure, which is then distinct from the previous three 60. (See Sec. 7.2 for further discussion).

It is convenient to parametrize the metric and almost complex structure as in Ex. 1:

$$
\begin{aligned}
& \eta^{w^{1}}=\eta^{4}+\tau^{w^{1}} \eta^{5}, \\
& d w^{i}=d x^{2 i+2}+\tau^{w^{i}} d x^{2 j+3}, \quad i, j=2,3 .
\end{aligned}
$$

Here, we have used the symbols $w^{i}$ instead of $z^{i}$, reserving the latter for complex coordinates in which $G_{(3)}$ is of type $(2,1)$ and primitive.

The condition that $G_{(3)}$ be of type $(0,3)$ results in the following constraints:

$$
\begin{aligned}
& \tau^{w^{i}}{ }_{j}=\tau \delta^{i}{ }_{j}, \quad \text { where } \quad-(h / f) \bar{\tau}_{\text {dil }}=\tau^{w^{1}}=\tau, \quad \text { and } \\
& \tau^{2}+m \tau+n=0, \quad \text { or equivalently, } \quad \tau=\frac{1}{2}\left(-m+i \sqrt{4 n-m^{2}}\right) .
\end{aligned}
$$

Here, we have chosen the root $\tau$ such that $\operatorname{Im} \tau>0$, for agreement of Eq. (7.5a) with $\operatorname{Im} \tau_{\text {dil }}=1 / g_{s}>0$. When Eqs. $(7.5 a, b)$ are satisfied,

$$
\frac{1}{(2 \pi)^{2} \alpha^{\prime}} G_{(3)}=2 f \eta^{\bar{w}^{1}} \wedge d \bar{w}^{2} \wedge d \bar{w}^{3},
$$

which is indeed of type $(0,3)$. Since this statement is independent of the Kähler moduli, the unfixed metric moduli are the five real Kähler moduli $V_{\mathrm{fib}}$ and $g_{\mathcal{B} w^{i} \bar{w}^{j}}(i, j=2,3)$, with $g_{\mathcal{B} w^{i} \bar{w}^{j}}=\left(g_{\mathcal{B} w^{j}} \bar{w}^{i}\right)^{*}$. The constraints $(7.5 a, \mathbb{Z})$ also imply that

$$
\mathcal{F}^{w^{1}}=2 h d \bar{w}^{2} \wedge d \bar{w}^{3}
$$

where $\mathcal{F}^{w^{1}}=\mathcal{F}^{4}+\tau^{w^{1}} \mathcal{F}^{5}$.

Now let us return to the supersymmetry conditions as originally formulated. The three independent complex structures satisfying conditions $(4.28 a, b)$ are related to the one just described via

$$
\begin{array}{ll}
\text { complex structure 1: } & \left(\eta^{z^{1}}, d z^{2}, d z^{3}\right)=\left(\eta^{w^{1}}, d \bar{w}^{2}, d \bar{w}^{3}\right) \\
\text { complex structure 2: } & \left(\eta^{z^{1}}, d z^{2}, d z^{3}\right)=\left(\eta^{w^{1}}, d w^{2}, d \bar{w}^{3}\right) \\
\text { complex structure 3: } & \left(\eta^{z^{1}}, d z^{2}, d z^{3}\right)=\left(\eta^{\bar{w}^{1}}, d \bar{w}^{2}, d w^{3}\right) .
\end{array}
$$


In these three complex structures,

$$
\mathcal{F}^{z^{1}}=2 h d z^{2} \wedge d z^{3}, \quad \mathcal{F}^{z^{1}}=2 h d \bar{z}^{2} \wedge d z^{3}, \quad \text { and } \quad \mathcal{F}^{z^{1}}=2 h d z^{2} \wedge d \bar{z}^{3}, \quad(7.9 a, b, c)
$$

respectively. In all three cases, $\mathcal{F}^{z^{1}}$ has no $(0,2)$ component. Therefore, the corresponding fibrations are holomorphic, and the complex structures are integrable, in agreement with Sec. 4.2. In constrast, the almost complex structure of the $w$ coordinates is not integrable.

Specialize to complex structure 1. Then we can write Eq. (7.9a) as

$$
d \eta^{z^{1}}=N d z^{2} \wedge d z^{3}, \text { where } \quad N=2 h .
$$

This is the familiar relation between the three left-invariant one-forms

$$
\eta^{z^{1}}=z^{1}+N z^{2} d z^{3}, \quad d z^{2}, \text { and } d z^{3}
$$

on the Iwasawa manifold.

The Iwasawa manifold can be defined as a coset of upper triangular matrices as follows. 30 Consider $\mathbb{C}^{3}$, presented as the space of upper triangular $3 \times 3$ matrices with ones along the diagonal,

$$
g_{N}\left(z^{1}, z^{2}, z^{3}\right)=\left(\begin{array}{ccc}
1 & z^{3} & -z^{1} / N \\
0 & 1 & z^{2} \\
0 & 0 & 1
\end{array}\right), \quad \text { where } \quad z^{1}, z^{2}, z^{3} \in \mathbb{C} .
$$

Here, $N$ is a fixed positive integer. Let $\mathcal{G}_{3}^{N}(\mathbb{C})$ deonte the corresponding group under matrix multiplication. For any $N$, this group is isomorphic to the complex three-dimensional Heisenberg group $\mathcal{H}_{3}(\mathbb{C})$. We can also define a discrete subgroup $\mathcal{G}_{3}^{N}(\Lambda)$ containing the matrices

$$
g_{N}(a, b, c)=\left(\begin{array}{ccc}
1 & c & -a / N \\
0 & 1 & b \\
0 & 0 & 1
\end{array}\right), \quad \text { where } a, b, c \in \Lambda,
$$

with $\Lambda=\mathbb{Z}+\tau \mathbb{Z} \subset \mathbb{C}$ a lattice parametrized by the complex modulus $\tau$. This subgroup has a natural action on $\mathcal{G}_{3}^{N}(\mathbb{C})$ by matrix multiplication. Consider the right-coset $\mathcal{M}^{N}=$ $\mathcal{G}_{3}^{N}(\mathbb{C}) / \mathcal{G}_{3}^{N}(\Lambda)$. The resulting identifications are

$$
\left(z^{1}, z^{2}, z^{3}\right) \cong\left(z^{1}+a-N b z^{3}, z^{2}+b, z^{3}+c\right)
$$

30 This description is taken directly from the twisted $T^{3}$ example in Sec. 2.2 of Ref. [四, with $\mathbb{R}^{3}$ replaced by $\mathbb{C}^{3}$. 
This quotient defines the $N$ th Iwasawa manifold.

The standard metric and Kähler form on the Iwasawa manifold are

$$
\begin{aligned}
d s_{\text {Iwasawa }}^{2} & =\left|\eta^{z^{1}}\right|^{2}+\left|d z^{2}\right|^{2}+\left|d z^{3}\right|^{2} \\
J_{\text {Iwasawa }} & =\frac{i}{2}\left(\eta^{z^{1}} \wedge \eta^{\bar{z}^{1}}+d z^{2} \wedge d \bar{z}^{2}+d z^{3} \wedge d \bar{z}^{3}\right) .
\end{aligned}
$$

However, in the orientifold example that we are considering, the metric and Kähler form are given by 3

$$
\begin{aligned}
d s_{6}{ }^{2} & =\frac{V_{\mathrm{fib}}}{\operatorname{Im} \tau}\left|\eta^{z^{1}}\right|^{2}+2 Z g_{\mathcal{B} z^{i} \bar{z}^{j}} d z^{i} d \bar{z}^{j}, \\
J & =\frac{i V_{\mathrm{fib}}}{2 \operatorname{Im} \tau} \eta^{z^{1}} \wedge \eta^{\bar{z}^{1}}+i Z g_{\mathcal{B} z^{i} \bar{z}^{j}} d z^{i} \wedge d \bar{z}^{j},
\end{aligned}
$$

a generalization of $(7.14 a, b)$ that includes arbitrary fiber and base Kähler structure, and also the warp factor $Z$.

Now let us turn to the NS $B$-field moduli. The constraints that follow from Eq. (3.52a) are

$$
\begin{array}{ll}
m b_{(2) 46}+b_{(2) 47}+b_{(2) 56}=0, & n b_{(2) 46}-b_{(2) 57}=0, \\
m b_{(2) 48}+b_{(2) 49}+b_{(2) 58}=0, & n b_{(2) 48}-b_{(2) 59}=0,
\end{array}
$$

or equivalently,

$$
b_{(2) w^{1} w^{i}}=b_{(2) \bar{w}^{1} \bar{w}^{i}}=0, \quad i=2,3 .
$$

Eq. (3.52b) gives the same constraints. The unlifted NS $B$-field moduli are the orthogonal components

$$
b_{(2) w^{1} \bar{w}^{i}}, \quad b_{(2) \bar{w}^{1} w^{i}}, \quad i=2,3 .
$$

From the kinetic terms $(3.54 a, b, d)$, the massless RR sector scalars are the nine uncharged fields

$$
\begin{aligned}
& \widetilde{c}_{(2) 67}, \quad \widetilde{c}_{(2) 89}, \quad \widetilde{c}_{(2) 69}-\widetilde{c}_{(2) 78}, \quad \widetilde{c}_{(2) \mu \nu} \leftrightarrow \widetilde{c}_{(6) 456789}, \\
& n \widetilde{c}_{(2) 68}+(m / 2)\left(\widetilde{c}_{(2) 69}+\widetilde{c}_{(2) 78}\right)+\widetilde{c}_{(2) 79}, \\
& \widetilde{c}_{(4) 5678}-\widetilde{c}_{(4) 4679}, \quad \widetilde{c}_{(4) 4789}-\widetilde{c}_{(4) 5689}, \\
& n \widetilde{c}_{(4) 4678}+(m / 2)\left(\widetilde{c}_{(4) 4679}+\widetilde{c}_{(4) 5678}\right)+\widetilde{c}_{(4) 5679}, \\
& \text { and } n \widetilde{c}_{(4) 4689}+(m / 2)\left(\widetilde{c}_{(4) 4789}+\widetilde{c}_{(4) 5689}\right)+\widetilde{c}_{(4) 5789} .
\end{aligned}
$$

31 In the conventions of this paper, $d s^{2}=g_{i \bar{\jmath}} d z^{i} d \bar{z}^{j}+g_{\bar{\imath} j} d \bar{z}^{i} d z^{j}=2 g_{i \bar{\jmath}} d z^{i} d \bar{z}^{j}$ (cf. App. A). 
The massive gauge bosons are those that couple to the other RR scalars. From $(3.54 a, b, 3)$, they lie in the $6 \mathrm{D}$ space spanned by

$$
\begin{aligned}
& (2 \pi)^{2} \alpha^{\prime}(f / h) v_{\mu}^{4}-c_{(2) 4 \mu}, \quad(2 \pi)^{2} \alpha^{\prime}(f / h) v_{\mu}^{5}-m c_{(2) 4 \mu}-c_{(2) 5 \mu}, \\
& (2 \pi)^{2} \alpha^{\prime}(f / h) b_{(2) 7 \mu}-\widetilde{c}_{(4) 456 \mu}, \quad(2 \pi)^{2} \alpha^{\prime}(f / h) b_{(2) 6 \mu}+m \widetilde{c}_{(4) 456 \mu}+\widetilde{c}_{(4) 457 \mu}, \\
& (2 \pi)^{2} \alpha^{\prime}(f / h) b_{(2) 9 \mu}-\widetilde{c}_{(4) 458 \mu}, \quad(2 \pi)^{2} \alpha^{\prime}(f / h) b_{(2)} 8 \mu+m \widetilde{c}_{(4) 458 \mu}+\widetilde{c}_{(4) 459 \mu} .
\end{aligned}
$$

The massless gauge bosons span the orthogonal 6D space, where orthogonality is defined with respect to the metric appearing in the gauge kinetic terms. (See Sec. 7.2 for further discussion in the dual O3 orientifold).

Finally, there are $6 M$ massless scalars $A_{I \alpha}, \Phi_{I}{ }^{m}$, and $M$ massless gauge bosons $A_{I \mu}$ from the D5 branes.

In summary, the massless bosonic fields are one graviton, $6+M$ vectors, and $18+6 M$ moduli. The moduli consist of

$$
\begin{gathered}
V_{\text {fib }}, 4 g_{\mathcal{B} z^{i} \bar{z}^{j}}, 4 \text { indep } b_{(2) \alpha m}, \\
9 \widetilde{c}_{(p)} \text { scalars, and } 6 M \text { D5 scalars. }
\end{gathered}
$$

These fields combine to form one $4 \mathrm{D} \mathcal{N}=3$ gravity multiplet, and $3+M$ vector multiplets.

The amount of unbroken supersymmetry is

$$
\mathcal{N}_{+}=1, \quad \mathcal{N}_{-}=2,
$$

in the notation of Sec. 4.2. The $\mathcal{N}_{+}=1$ supersymmetry corresponds to complex structure 1 (7.8a), with the modulus $\tau_{1}=\tau$ of $\operatorname{Im} \tau_{1}>0$ appearing in $\eta^{z^{1}}$ (cf. Eq. (4.26a)). The $\mathcal{N}_{-}=2$ supersymmetries correspond to complex structures 2 and $3(7.8 b, \mathrm{~d})$, with the modulus $\tau_{1}=\bar{\tau}$ of $\operatorname{Im} \tau_{1}<0$ appearing in $\eta^{z^{1}}$.

\subsection{Dual O3 Orientifold}

For the O3 orientifold with internal $T^{6}$, choices of flux preserving $4 \mathrm{D} \mathcal{N}=3$ supersymmetry were first discussed in Ref. [60]. The requirement for $\mathcal{N}=3$ supersymmetry is

$G_{(3)}^{\prime} \quad(2,1)$ and primitive w.r.t. to three independent complex structures.

In contrast, from the equations of motion alone, without imposing any supersymmetry conditions, one obtains the condition that the flux be ISD: $*_{6} G_{(3)}^{\prime}=i G_{(3)}^{\prime}$. As has already 
been mentioned in Footnote 21, the space of ISD forms includes not only primitive $(2,1)$ forms, but also nonprimitive $(1,2)$ forms $J \wedge \omega^{(0,1)}$, where $\omega^{(0,1)}$ is a $(0,1)$ form, as well as $(0,3)$ forms. The condition $(\mathbf{7 . 2 1})$ is equivalent to 60]

$$
G_{(3)}^{\prime} \quad(0,3) \text { for some choice of complex structure. }
$$

The complex structure in this last condition is a fourth complex structure, independent of the three complex structures of the previous condition. 32

To construct a large class of $\mathcal{N}=3$ backgrounds, let us write

$$
\frac{1}{(2 \pi)^{2} \alpha^{\prime}} G_{(3)}^{\prime}=2 \lambda d \bar{w}^{1} \wedge d \bar{w}^{2} \wedge d \bar{w}^{3}
$$

where $\lambda$ is a positive real number, and where the $T^{6}$ factorizes as $T^{2} \times T^{2} \times T^{2}$ with respect to complex structure in the following way:

$$
\begin{aligned}
& w^{i}=x^{i}+\tau^{\prime} y^{i}, \quad i=1,2,3, \\
& (h / f) \tau_{\text {dil }}{ }^{\prime}=-\bar{\tau}^{\prime}, \quad f, h \in \mathbb{N} .
\end{aligned}
$$

The coordinates $x^{i}, y^{i}$ are related to those used elsewhere in this paper by

$$
\left(x^{1}, y^{1}, x^{2}, y^{2}, x^{3}, y^{3}\right)_{\text {here }}=\left(-x^{5}, x^{4}, x^{6}, x^{7}, x^{8}, x^{9}\right)_{\text {rest of paper }}
$$

We adopt this notation here and similar notation in Sec. 8.2 since it allows equations involving cyclic permutation of the $x^{i}, y^{i}$ to be written succinctly.

When expanded in real coordinates, the complex flux becomes

$$
\begin{aligned}
\frac{1}{(2 \pi)^{2} \alpha^{\prime}} G_{(3)}^{\prime} & =2 \lambda\left(d x^{1} \wedge d x^{2} \wedge d x^{3}+\bar{\tau}^{\prime}\left(d x^{1} \wedge d x^{2} \wedge d y^{3}+\text { cyc. perms. of } 123\right)\right. \\
& \left.+\bar{\tau}^{\prime 2}\left(d x^{1} \wedge d y^{2} \wedge d y^{3}+\text { cyc. perms. of } 123\right)+\bar{\tau}^{\prime 3} d y^{1} \wedge d y^{2} \wedge d y^{3}\right)
\end{aligned}
$$

32 In fact, we could have also rephrased the supersymmetry conditions for the $\mathcal{N}=2$ examples. In each example of Sec. 6 , there exists a complex structure such that $G_{(3)}, \widetilde{G}_{(3)}$, or $G_{(3)}^{\prime}$ (whichever is appropriate) is of the form $J \wedge \omega^{(0,1)}$. However, unlike the $\mathcal{N}=4 \rightarrow \mathcal{N}=3$ case, where the conditions (7.21) and (7.22) are truly equivalent, in the $\mathcal{N}=4 \rightarrow \mathcal{N}=2$ case, this rephrasing of the supersymmetry conditions seems to involve the assumption that $m_{1}=m_{2}$ for the two massive gravitini. 
If, in addition, we assume as part of our ansatz that $\tau^{\prime}$ satisfies the quadratic equation

$$
P\left(\tau^{\prime}\right)=l \tau^{\prime 2}+m \tau^{\prime}+n=0, \quad l, m, n \in \mathbb{Z}, \quad l>0,
$$

then Eq. (7.26) reduces to an expression linear in $\bar{\tau}^{\prime}$. From Eqs. (7.24a,b), we can then read off the RR and NS fluxes. It is possible to show from the quantization condition (5.4) that by redefinitions of $l, m, n$, and $\lambda$, we can take $\lambda=f l$. Then,

$$
\begin{aligned}
& \frac{1}{(2 \pi)^{2} \alpha^{\prime}} F_{(3)}^{\prime}=2 f\left(l d x^{1} \wedge d x^{2} \wedge d x^{3}-n\left(d x^{1} \wedge d y^{2} \wedge d y^{3}+\text { cyc. perms. of } 123\right)\right. \\
& \left.\quad+(m n / l) d y^{1} \wedge d y^{2} \wedge d y^{3}\right) \\
& \frac{1}{(2 \pi)^{2} \alpha^{\prime}} H_{(3)}^{\prime}=2 h\left(l\left(d x^{1} \wedge d x^{2} \wedge d y^{3}+\text { cyc. perms. of } 123\right)\right. \\
& \left.-m\left(d x^{1} \wedge d y^{2} \wedge d y^{3}+\text { cyc. perms. of } 123\right)+\left(m^{2} / l-n\right) d y^{1} \wedge d y^{2} \wedge d y^{3}\right) \cdot(7
\end{aligned}
$$

The contribution to the Gauss's law constraint (3.15) from this choice of flux is

$$
N_{\text {flux }}=4 f h\left(4 l n-m^{2}\right) .
$$

Not all choices of $(l, m, n)$ are inequivalent. From change of lattice basis on the $T^{6}$, there is an $S L(2, \mathbb{Z})_{\tau^{\prime}}$ identification of the complex structure modulus $\tau^{\prime}$. Modulo identifications, we can assume that $\tau^{\prime}$ lies in the fundamental domain of $S L(2, \mathbb{C})$,

$$
\tau^{\prime} \in \mathcal{F}_{0}=\left\{\tau^{\prime} \in \mathbb{C}\left|-\frac{1}{2} \leq \operatorname{Re} \tau^{\prime}<\frac{1}{2},\right| \tau^{\prime} \mid \geq 1\right\} .
$$

From Eq. (7.27), we have

$$
\tau^{\prime}=\frac{1}{2 l}\left(-m+i \sqrt{4 l n-m^{2}}\right), \quad\left|\tau^{\prime}\right|=\sqrt{n / l},
$$

so the condition (7.30) becomes

$$
n>l \geq|m| \text { and } \quad 4 l n>m^{2} .
$$

The possible values of $(f, h, l, m, n)$ are strongly constrained by $0 \leq N_{\text {flux }} \leq 32$ and the fact that $m^{2} \equiv 0,1(\bmod 4)$. For $(f, h)=(1,1)$, in addition to the $\mathcal{N}=4$ solution without flux, there are just four choices of $(l, m, n)$ satisfying the inequalities (7.32):

$$
\begin{aligned}
& (l, m, n)=(1,1,1), \quad N_{\text {flux }}=12, \quad 2 M=20, \quad \tau^{\prime}=e^{2 \pi i / 3}, \\
& (l, m, n)=(1,0,1), \quad N_{\text {flux }}=16, \quad 2 M=16, \quad \tau^{\prime}=i, \\
& (l, m, n)=(1,1,2), \quad N_{\text {flux }}=28, \quad 2 M=4, \quad \tau^{\prime}=\frac{1}{2}(-1+i \sqrt{7}), \\
& (l, m, n)=(1,0,2), \quad N_{\text {flux }}=32, \quad 2 M=0, \quad \tau^{\prime}=i \sqrt{2} .
\end{aligned}
$$


Allowing arbitrary values of $(f, h)$, there are four additional choices. For $(f, h)=(2,1)$ or $(1,2)$, we can take

$$
\begin{aligned}
& (l, m, n)=(1,1,1), \quad N_{\text {flux }}=24, \quad 2 M=8, \quad \tau^{\prime}=e^{2 \pi i / 3}, \\
& (l, m, n)=(1,0,1), \quad N_{\text {flux }}=32, \quad 2 M=0, \quad \tau^{\prime}=i .
\end{aligned}
$$

Note that the flux $(7.28 a, b)$ is correctly quantized with $F_{(3)}^{\prime}, H_{(3)}^{\prime} \in(2 \pi)^{2} \alpha^{\prime} H^{3}\left(T^{6}, 2 \mathbb{Z}\right)$ in all of four cases.

If the complex structure and axion-dilaton are deformed from $\tau^{i}{ }_{j}=\tau^{\prime} \delta^{i}{ }_{j}$ and $(h / f) \tau_{\text {dil }}{ }^{\prime}=\tau^{\prime}$, with $\tau^{\prime}$ given by the values in $(7.33 a, b)$, then the complex flux is no longer of type $(0,3)$. On the other hand, the flux is still $(0,3)$ for arbitrary choice of Kähler moduli. So, the metric moduli are the nine real degrees of freedom $g_{w^{i} \bar{w}^{j}}^{\prime}$.

From the kinetic terms (5.15), the space of massless RR scalars is spanned by the nine uncharged scalars

$$
\begin{aligned}
& n c_{(4) x^{1} y^{1} x^{2} x^{3}}^{\prime}+c_{(4) x^{1} y^{1} y^{2} y^{3}}^{\prime}+(m / 2)\left(c_{(4) x^{1} y^{1} x^{2} y^{3}}^{\prime}+c_{(4) x^{1} y^{1} y^{2} x^{3}}^{\prime}\right), \\
& c_{(4) x^{1} y^{1} x^{2} y^{2}}^{\prime}, \quad c_{(4) x^{1} y^{1} x^{2} y^{3}}^{\prime}-c_{(4) x^{1} y^{1} y^{2} x^{3}}^{\prime}, \quad \text { and } \quad \text { cyc. perms. of } 123 \text {. }
\end{aligned}
$$

This is equivalent to the space spanned by the RR scalars of Hodge type $(2,2)$,

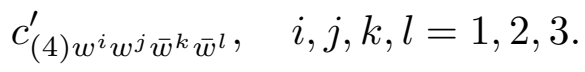

The massive gauge bosons are those that couple to the other RR scalars. From (5.15), they lie in the $6 \mathrm{D}$ space spanned by

$$
(f / h) n b_{(2) x^{i} \mu}^{\prime}-m c_{(2) x^{i} \mu}^{\prime}-c_{(2) y^{i} \mu}^{\prime}, \quad \text { and } \quad(f / h) b_{(2) y^{i} \mu}^{\prime}+c_{(2) x^{i} \mu}^{\prime}, \quad i=1,2,3 .
$$

In terms of the complex gauge bosons (5.16), this is the space spanned by $d_{w^{i} \mu}^{\prime}$, for $i=$

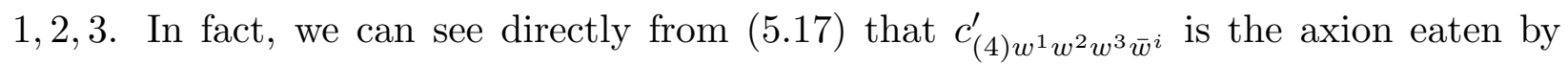
$d_{w^{i} \mu}^{\prime}$ [60]. The massless bosons are the linear combinations orthogonal to this space, where orthogonality is defined with respect to the metric appearing in the gauge kinetic terms. In the $\left(d_{w^{i} \mu}^{\prime}, d_{\bar{w}^{i} \mu}^{\prime}, \bar{d}_{w^{i} \mu}^{\prime}, \bar{d}_{\bar{w}^{i} \mu}^{\prime}\right)$ basis of complex gauge bosons (5.16), the latter is proportional to

$$
\frac{1}{2}\left(\begin{array}{ll}
0 & 1 \\
1 & 0
\end{array}\right) \bar{d}^{d^{\prime}} \quad \otimes \quad\left(\begin{array}{cc}
0 & g_{6}^{\prime w^{i}} \bar{w}^{j} \\
g_{6}^{\prime \bar{w}^{i} w^{j}} & 0
\end{array}\right){ }_{\bar{w}^{i}}^{w^{i}}
$$

Therefore, the six massless gauge bosons are (the real and imaginary parts of) $d_{\bar{w}^{i} \mu}^{\prime}$, for $i=1,2,3$ 60. 
Finally, there are $6 M$ massless scalars $\Phi_{I}^{\prime m}$, and $M$ massless gauge bosons $A_{I \mu}^{\prime}$ from the D3 branes.

In summary, the massless fields are one graviton, $6+M$ vectors, and $18+6 M$ moduli. The moduli consist of

$$
9 g_{\mathcal{B} w^{i} \bar{w}^{j}}, \quad 9 \widetilde{c}_{(p)} \text { scalars, and } 6 M \text { D3 scalars. }
$$

These fields combine to form one $4 \mathrm{D} \mathcal{N}=3$ gravity multiplet, and $3+M$ vector multiplets.

From Ref. [77], the moduli space of the $\mathcal{N}=3$ theory is completely determined by the number $n_{V}$ of vector multiplets. It is the coset

$$
\mathcal{M}=\frac{U\left(3, n_{V}\right)}{U(3) \times U\left(n_{V}\right)}
$$

up to discrete identifications, where, in this example, $n_{V}=3+M$. For the parametrization of the coset in terms of the above moduli, see Ref. [60].

\subsection{T-Duality Map}

For the $\mathrm{O} 3$ orientifold of Sec. 7.2 , the metric is

$$
d s_{6}^{\prime 2}=2 g_{w^{i} \bar{w}^{j}} d w^{i} d \bar{w}^{j}
$$

with $g_{w^{i} \bar{w}^{j}}$ constrained only to be Hermitian. We can alternatively write this metric in the fibration form (5.18),

$$
d s_{6}^{\prime 2}=\frac{V_{\text {fib }}^{\prime}}{\operatorname{Im} \tau^{\prime}}\left|d w^{1}+a^{\prime w^{1}}\right|^{2}+2 g_{\mathcal{B} w^{i} \bar{w}^{j}} d w^{i} d \bar{w}^{j},
$$

where the components of $a^{\prime \alpha}$ are holomorphically constrained,

$$
a^{\prime w^{1}}=a^{\prime w^{1}} w^{i} d w^{i}, \quad \text { and } \quad a^{\prime \bar{w}^{1}}=a^{\prime \bar{w}^{1}} \bar{w}^{i} d \bar{w}^{i}, \quad i=2,3,
$$

and where

$$
\begin{aligned}
V_{\mathrm{fib}}^{\prime} & =2 g_{w^{1} \bar{w}^{1}} \operatorname{Im} \tau^{\prime}, \\
g_{\mathcal{B} w^{i} \bar{w}^{j}} & =g_{w^{i} \bar{w}^{j}}-g_{w^{1} \bar{w}^{1}} a^{\prime w^{1}}{ }_{w^{i}} a^{\bar{w}^{1}}{ }_{\bar{w}^{j}}, \quad i, j=2,3 .
\end{aligned}
$$

Here, from Eqs. (7.24a) and (7.25), the complex coordinates in the O3 orientifold are

$$
w^{1}=-x^{5}+\tau^{\prime} x^{4} \quad \text { and } \quad w^{i}=x^{2 i+2}+\tau^{\prime} x^{2 i+3}, \quad i=2,3 .
$$


Applying the T-duality map of Sec. 5.2, we find agreement between Secs. 7.1 and 7.2, provided that we identify $\tau=\tau^{\prime}$. For example, the map between complex components of $a^{\prime \alpha}$ and $b_{(2) \alpha}$ is

$$
b_{(2) w^{1}}=a^{\prime \bar{w}^{1}} /\left(\tau^{\prime}-\bar{\tau}^{\prime}\right) \quad \text { and } \quad b_{(2) \bar{w}^{1}}=-a^{\prime w^{1}} /\left(\tau^{\prime}-\bar{\tau}^{\prime}\right),
$$

so, the constraints $(\sqrt{7.16})$ and $(7.40 a)$ agree. Here, $\eta^{w^{1}}=\eta^{4}+\tau \eta^{5}$ for the O5 orientifold, from which the relation between $b_{(2) w^{1}}, b_{(2) \bar{w}^{1}}$ and $b_{(2) 4}, b_{(2) 5}$ is

$$
b_{(2) w^{1}}=\frac{1}{\tau-\bar{\tau}}\left(b_{(2) 5}-\bar{\tau} b_{(2) 4}\right) \quad \text { and } \quad b_{(2) w^{1}}=-\frac{1}{\tau-\bar{\tau}}\left(b_{(2) 5}-\tau b_{(2) 4}\right) .
$$

Similarly, using the underscore notation of Sec. 5.2,

$$
\begin{aligned}
\mathcal{F}_{m n}^{w^{1}} & =\mathcal{F}_{m n}^{4}+\tau \mathcal{F}_{m n}^{5}=H_{(3) \underline{4 m n}}^{\prime}+\tau^{\prime} H_{(3)}^{\prime} \underline{5 m n} \\
& =-\left(\tau^{\prime}-\bar{\tau}^{\prime}\right) H_{(3) \underline{\bar{w}^{1} m n}}^{\prime}=(h / f) G_{(3) \underline{\bar{w}^{1} m n}}^{\prime},
\end{aligned}
$$

from which Eq. (7.7) follows.

\section{8. $\mathcal{N}=1$ Example}

\subsection{Example 5: $\mathcal{N}_{+}=1, \mathcal{N}_{-}=0$}

Consider the choice of fibration curvature and background flux

$$
\begin{aligned}
& \mathcal{F}^{4}=2 h\left(d x^{6} \wedge d x^{8}-d x^{6} \wedge d x^{9}-d x^{7} \wedge d x^{8}+d x^{7} \wedge d x^{9}\right), \\
&-\mathcal{F}^{5}=2 h\left(d x^{6} \wedge d x^{8}-d x^{6} \wedge d x^{9}-d x^{7} \wedge d x^{8}+2 d x^{7} \wedge d x^{9}\right), \\
& \frac{1}{(2 \pi)^{2} \alpha^{\prime}} F_{(3)}^{\mathrm{bg} 1}=2 f \eta^{5} \wedge d x^{6} \wedge d x^{8}-2 f \eta^{4} \wedge d x^{7} \wedge d x^{9},
\end{aligned}
$$

with $F_{(1)}=H_{(3)}=0$. This choice gives a contribution to the Gauss's law constraint

$$
N_{\text {flux }}=12 f h,
$$

so that Eq. (3.15) becomes

$$
2 M+12 f h=32 .
$$

As discussed in Sec. 3.2, $N_{\text {flux }}$ is nonnegative. So, $f$ and $h$ have the same sign, and the possible values of $(f, h)$ are $(1,1),(2,1)$, and $(1,2)$, corresponding to $2 M=20,8$, and 8 D5 branes, respectively. 
Define an almost complex structure as in Eq. (6.5). Then, using the decomposition (4.21), it is possible to show that the base $T^{4}$ decomposes as $T^{4} \rightarrow T^{2} \times T^{2}$ with respect to complex structure:

$$
\tau^{i}{ }_{j}=\tau \delta^{i}{ }_{j}, \quad i, j=2,3
$$

where

$$
-1 / \tau_{1}=\tau=(h / f) \tau_{\text {dil }}=e^{2 \pi i / 3}
$$

When these constraints are satisfied, the complex three-form flux (4.28d) is

$$
G_{(3)}=-(2 i f / \sqrt{3})\left(\eta^{z^{1}} \wedge d z^{2} \wedge d \bar{z}^{3}+\eta^{z^{1}} \wedge d \bar{z}^{2} \wedge d z^{3}+\eta^{\bar{z}^{1}} \wedge d z^{2} \wedge d z^{3}\right)
$$

The primitivity condition (4.28b) then constrains $J$ to be of the form

$$
J=\frac{i}{2 \operatorname{Im} \tau_{1}} V_{\mathrm{fib}} \eta^{z^{1}} \wedge \eta^{\bar{z}^{1}}+\frac{1}{2 \operatorname{Im} \tau}\left(i V_{2} d z^{2} \wedge d \bar{z}^{2}+i V_{3} d z^{3} \wedge d \bar{z}^{3}-2 V_{(23)} \operatorname{Im}\left(d z^{2} \wedge d \bar{z}^{3}\right)\right)
$$

corresponding to the base metric

$$
d s_{\mathcal{B}}^{2}=\frac{1}{|\operatorname{Im} \tau|}\left(V_{2} d z^{2} d \bar{z}^{2}+V_{3} d z^{3} d \bar{z}^{3}+V_{(23)} \operatorname{Re}\left(d z^{2} d \bar{z}^{3}\right)\right)
$$

From Eqs. (3.52a,, ), the constraints on NS $B$-field moduli are

$$
\begin{aligned}
0= & 2 h\left(b_{(2) 4 m}-b_{(2) 5 m}\right) d x^{m} \wedge\left(d x^{6} \wedge d x^{8}-d x^{6} \wedge d x^{9}-d x^{7} \wedge d x^{8}+d x^{7} \wedge d x^{9}\right) \\
& \quad-2 h b_{(2) 5 m} d x^{m} \wedge d x^{7} \wedge d x^{9} \\
0= & -2 f b_{(2) 4 m} d x^{m} \wedge d x^{7} \wedge d x^{9}+2 f b_{(2) 5 m} \wedge d x^{m} \wedge d x^{6} \wedge d x^{8} .
\end{aligned}
$$

Together, these constraints imply

$$
\begin{aligned}
& b_{(2) 47}=b_{(2) 49}=b_{(2) 56}=b_{(2) 58}=0 \\
& b_{(2) 46}=b_{(2) 57}, \quad \text { and } \quad b_{(2) 48}=b_{(2) 59} .
\end{aligned}
$$

So, there are two unconstrained NS $B$-field moduli,

$$
b_{(2) 46}+b_{(2) 57} \quad \text { and } \quad b_{(2) 48}+b_{(2)} 59 .
$$

From the kinetic terms $(3.54 a, b, d)$, the massless RR sector scalars are the uncharged fields

$$
\begin{aligned}
& \widetilde{c}_{(2) 67}, \quad \widetilde{c}_{(2) 89}, \quad \widetilde{c}_{(2) 69}-\widetilde{c}_{(2) 78}, \quad \widetilde{c}_{(2) \mu \nu} \leftrightarrow \widetilde{c}_{(6) 456789}, \\
& \widetilde{c}_{(4) 4678}+\widetilde{c}_{(4) 5679}, \quad \text { and } \quad \widetilde{c}_{(4) 5789}+\widetilde{c}_{(4) 4689} .
\end{aligned}
$$


The massive gauge bosons are those that couple to the other RR scalars. From $(3.54 a, b, d)$, they lie in the 9D space spanned by

$$
\begin{aligned}
& (2 \pi)^{2} \alpha^{\prime}(f / h) v_{\mu}^{4}-c_{(2) 5 \mu}, \quad v_{\mu}^{5}, \quad c_{(2) 4 \mu}-c_{(2) 5 \mu}, \\
& (2 \pi)^{2} \alpha^{\prime}(f / h) b_{(2) 6 \mu}-\widetilde{c}_{(4) 456 \mu}, \quad b_{(2) 7 \mu}, \quad \widetilde{c}_{(4) 456 \mu}+\widetilde{c}_{(4) 457 \mu}, \\
& (2 \pi)^{2} \alpha^{\prime}(f / h) b_{(2) 8 \mu}-\widetilde{c}_{(4) 458 \mu}, \quad b_{(2) 9 \mu}, \quad \widetilde{c}_{(4) 458 \mu}+\widetilde{c}_{(4) 459 \mu} .
\end{aligned}
$$

The massless bosons are the three linear combinations orthogonal to this space, where orthogonality is defined with respect to the metric appearing in the gauge kinetic terms. (See Sec. 8.2 for further discussion in the dual O3 orientifold).

Finally, there are $6 M$ massless scalars $A_{I \alpha}, \Phi_{I}^{m}$, and $M$ massless gauge bosons $A_{I \mu}$ from the D5 branes.

In summary, the massless fields are one graviton, $3+M$ vectors, and $6+6 M$ moduli. The moduli consist of

$$
\begin{aligned}
& V_{\mathrm{fib}}, V_{2}, V_{3}, V_{(23)}, 2 \text { indep } b_{(2) \alpha m}, \\
& 6 \widetilde{c}_{(p)} \text { scalars, and } 6 M \text { D5 scalars. }
\end{aligned}
$$

These fields combine to form one $4 \mathrm{D} \mathcal{N}=1$ gravity multiplet, $3+3 M$ chiral multiplets, and $3+M$ vector multiplets.

The amount of unbroken supersymmetry is

$$
\mathcal{N}_{+}=1, \quad \mathcal{N}_{-}=0
$$

in the notation of Sec. 4.2. To verify this, first note that the complex structure modulus of the fiber is $\tau_{1}$, with $\operatorname{Im} \tau_{1}>0$ from (8.5). Therefore, the supersymmetry is of + type. Since the base factorizes as $T_{\{67\}}^{2} \times T_{\{89\}}^{2}$ with respect to complex structure, we can define chirality operators $\widetilde{\gamma}_{(2)}=\widetilde{\gamma}_{2}{ }^{2}$ on $T_{\{67\}}^{2}$ and $\widetilde{\gamma}_{(3)}=-\widetilde{\gamma}_{3}{ }^{3}$ on $T_{\{89\}}^{2}$, where the indices 2 and 3 are holomorphic $z^{2}$ and $z^{3}$ indices. 33 Then, since complex structure moduli of $T_{\{67\}}^{2}$ and $T_{\{89\}}^{2}$ are $\tau$, with $\operatorname{Im} \tau>0$ from $(8.5)$, there is one independent negative chirality $6 \mathrm{D}$ Killing spinor $\chi$. It is of the form

$$
\chi=\zeta_{+}^{\mathrm{fib}} \otimes \zeta^{(2)} \otimes \zeta^{(3)},
$$

where $\widetilde{\gamma}_{(2)} \zeta^{(2)}=\zeta^{(2)}$ and $\widetilde{\gamma}_{(3)} \zeta^{(3)}=-\zeta^{(2)}$. The three factors in Eq. (8.15) are on equal footing in the sense that $\widetilde{\gamma}_{i}^{i}$ (no sum) $=+1$ in all three cases.

33 Here, the relative minus sign is necessary for chiralities to be multiplicative, that is, for $\widetilde{\gamma}_{\mathcal{B}}=\widetilde{\gamma}_{(2)} \widetilde{\gamma}_{(3)}$. In the conventions of this paper, $\widetilde{\gamma}_{\mathcal{B}}=g_{\mathcal{B}}^{1 / 2} \widetilde{\gamma}^{6789}$ (cf. Eq. (A.17)), which equivalent to $\widetilde{\gamma}_{\mathcal{B}}=-\widetilde{\gamma}_{2}{ }^{2}{ }_{3}{ }^{3}$ when expressed in terms of holomorphic coordinates. 


\subsection{Dual O3 Orientifold}

In this subsection, it is convenient to use the notation

$$
\left(x^{1}, y^{1}, x^{2}, y^{2}, x^{3}, y^{3}\right)_{\text {here }}=\left(x^{4}, x^{5}, x^{6}, x^{7}, x^{8}, x^{9}\right)_{\text {rest of paper }}
$$

In this notation, consider the $\mathrm{O} 3$ orientifold with internal $T^{6}$ and flux

$$
\begin{gathered}
\frac{1}{(2 \pi)^{2} \alpha^{\prime}} F_{(3)}^{\prime}=a^{0} d x^{1} \wedge d x^{2} \wedge d x^{3}+a\left(d x^{1} \wedge d x^{2} \wedge d y^{3}+\right.\text { cyc. perms. of 123) } \\
-b\left(d x^{1} \wedge d y^{2} \wedge d y^{3}+\text { cyc. perms. of } 123\right)+b_{0} d y^{1} \wedge d y^{2} \wedge d y^{3} \\
\frac{1}{(2 \pi)^{2} \alpha^{\prime}} H_{(3)}^{\prime}=c^{0} d x^{1} \wedge d x^{2} \wedge d x^{3}+c\left(d x^{1} \wedge d x^{2} \wedge d y^{3}+\right.\text { cyc. perms. of 123) } \\
-d\left(d x^{1} \wedge d y^{2} \wedge d y^{3}+\text { cyc. perms. of } 123\right)+d_{0} d y^{1} \wedge d y^{2} \wedge d y^{3}
\end{gathered}
$$

This class of fluxes was analyzed in Sec. 4.1 of Ref. [11]. Let us quickly review that analysis. The $(2,1)$ constraint on the complex flux $G_{(3)}^{\prime}$ implies that

$$
\tau^{\prime i}{ }_{j}=\tau^{\prime} \delta^{i}{ }_{j},
$$

that is, $T^{6} \rightarrow T^{2} \times T^{2} \times T^{2}$ with respect to complex structure, where $\tau^{\prime}$ and $\tau_{\text {dil }}^{\prime}$ satisfy

$$
\begin{aligned}
& P_{1}\left(\tau^{\prime}\right) \equiv a^{0} \tau^{\prime 3}-3 a \tau^{\prime 2}-3 b \tau^{\prime}-b_{0}=0, \\
& P_{2}\left(\tau^{\prime}\right) \equiv c^{0} \tau^{\prime 3}-3 c \tau^{\prime 2}-3 d \tau^{\prime}-d_{0}=0,
\end{aligned}
$$

and

$$
\left(a^{0}-\tau_{\text {dil }}{ }^{\prime} c^{0}\right) \tau^{\prime 2}-2\left(a-\tau_{\text {dil }}{ }^{\prime} c\right) \tau^{\prime}-\left(b-\tau_{\text {dil }}{ }^{\prime} d\right)=0 .
$$

A solution exists only if

$$
P_{1}\left(\tau^{\prime}\right)=2\left(f \tau^{\prime}+g\right) P\left(\tau^{\prime}\right), \quad P_{2}\left(\tau^{\prime}\right)=2\left(h \tau^{\prime}+k\right) P\left(\tau^{\prime}\right)
$$

where $P\left(\tau^{\prime}\right)$ is a quadratic polynomial of the form (7.27), and $f, g, h, k \in \mathbb{Z}$. In Eq. (8.20), we have added factors of 2 compared to the corresponding equation in Ref. [11], in order to automatically take into account the $2 \mathbb{Z}$ quantization condition on the fluxes. The relations

$$
\begin{aligned}
2(f m+g l) & =-3 a, \quad 2(h m+k l)=-3 c \\
2(f n+g m) & =-3 b, \quad 2(h n+k m)=-3 d,
\end{aligned}
$$


give consistency conditions modulo 3, and the flux contribution to the Gauss's law constraint $(5.1 e)$ is

$$
N_{\text {flux }}=\frac{4}{3}(f k-g h)\left(m^{2}-4 l n\right) .
$$

It can be shown from Eq. (8.21) that $N_{\text {flux }}$ is divisible by 12 .

Ref. [1] went on to consider the particular example

$$
\begin{gathered}
\left(a^{0}, a, b, b_{0}\right)=(2,0,0,2), \quad\left(c^{0}, c, d, d_{0}\right)=(2,-2,-2,-4), \\
(f, g, h, k, l, m, n)=(1,-1,1,2,1,1,1) .
\end{gathered}
$$

In App. H, we prove that this is the unique choice of supersymmetric flux in the class $(8.17 a, b)$ with the minimum value $N_{\text {flux }}=12$ (and $2 \mathrm{M}=20$ D3 branes), modulo $S L(2, \mathbb{Z})_{\tau^{\prime}} \times S L(2, \mathbb{Z})_{\tau_{\mathrm{dil}^{\prime}}}$ equivalences 34 This choice gives

$$
\tau^{\prime}=\tau_{\text {dil }}^{\prime}=e^{2 \pi i / 3}
$$

The only other value of $N_{\text {flux }} \leq 32$ such that 12 divides $N_{\text {flux }}$ is $N_{\text {flux }}=24$ (with $2 \mathrm{M}=$ 8 D3 branes). By arguments analogous to those in App. $\mathrm{H}$, one can show that in this case there are two distinct choices of flux modulo equivalences: one choice differs from Eqs. (8.23a,b,c) by $f, g, \tau_{\text {dil }}{ }^{\prime} \rightarrow 2 f, 2 g, 2 \tau_{\text {dil }}{ }^{\prime}$, the other differs by $h, k, \tau_{\text {dil }}{ }^{\prime} \rightarrow 2 h, 2 k, \tau_{\text {dil }}{ }^{\prime} / 2$. So, in the entire class (8.17a,b), there are just three inequivalent choices of supersymmetric flux.

The three possibilities are summarized by

$$
\begin{gathered}
\frac{1}{(2 \pi)^{2} \alpha^{\prime}} F_{(3)}^{\prime}=2 f\left(d x^{1} \wedge d x^{2} \wedge d x^{3}+d y^{1} \wedge d y^{2} \wedge d y^{3}\right) \\
\frac{1}{(2 \pi)^{2} \alpha^{\prime}} H_{(3)}^{\prime}=2 h d x^{1} \wedge d x^{2} \wedge d x^{3}-2 h\left(d x^{1} \wedge d x^{2} \wedge d y^{3}+\text { cyc. perms. of } 1\right. \\
+2 h\left(d x^{1} \wedge d y^{2} \wedge d y^{3}+\text { cyc. perms. of } 123\right)-4 h d y^{1} \wedge d y^{2} \wedge d y^{3} \\
N_{\text {flux }}=12 f h, \quad \tau^{\prime}=(h / f) \tau_{\text {dil }}{ }^{\prime}=e^{2 \pi i / 3}
\end{gathered}
$$

where $(f, h)=(1,1),(2,1)$, or $(1,2)$.

The complex three-form flux is

$$
G_{(3)}^{\prime}=\left(2 i f e^{-2 \pi i / 3} / \sqrt{3}\right)\left(d z^{1} \wedge d z^{2} \wedge d \bar{z}^{3}+d z^{1} \wedge d \bar{z}^{2} \wedge d z^{3}+d \bar{z}^{1} \wedge d z^{2} \wedge d z^{3}\right) .
$$

34 This was suggested, but not proven in Ref. [11]. 
The primitivity condition (5.7) implies that $J^{\prime}$ is of the form

$$
\begin{aligned}
J^{\prime}= & \frac{1}{2\left|\operatorname{Im} \tau^{\prime}\right|}\left(i V_{1}^{\prime} d z^{1} \wedge d \bar{z}^{1}+i V_{2}^{\prime} d z^{2} \wedge d \bar{z}^{2}+i V_{3}^{\prime} d z^{3} \wedge d \bar{z}^{3}\right. \\
& \left.-2 V_{(12)}^{\prime} \operatorname{Im}\left(d z^{1} \wedge d \bar{z}^{2}\right)-2 V_{(23)}^{\prime} \operatorname{Im}\left(d z^{2} \wedge d \bar{z}^{3}\right)-2 V_{(31)}^{\prime} \operatorname{Im}\left(d z^{3} \wedge d \bar{z}^{1}\right)\right)
\end{aligned}
$$

where $z^{i}=x^{i}+\tau y^{i}$. In real coordinates, this becomes

$$
\begin{aligned}
J^{\prime}= & v_{1}^{\prime} d x^{1} \wedge d y^{1}+v_{2}^{\prime} d x^{2} \wedge d y^{2}+v_{3}^{\prime} d x^{3} \wedge d y^{3} \\
& +v_{(12)}^{\prime}\left(d x^{1} \wedge d y^{2}-d y^{1} \wedge d x^{2}\right)+(12 \rightarrow 23)+(12 \rightarrow 31),
\end{aligned}
$$

where $v_{i}^{\prime}=V_{i}^{\prime} \operatorname{sign} \tau^{\prime}$ and $v_{(i j)}^{\prime}=V_{(i j)}^{\prime} \operatorname{sign} \tau^{\prime}$. The metric is

$$
d s_{6}{ }^{2}=\frac{1}{\left|\operatorname{Im} \tau^{\prime}\right|}\left(\sum_{i=1}^{3} V_{i}^{\prime} d z^{i} d \bar{z}^{i}+\sum_{\substack{i, j=1 \\ i \neq j}}^{3} V_{(i j)}^{\prime} d z^{i} d \bar{z}^{j}\right),
$$

where $V_{(i j)}^{\prime}=V_{(j i)}^{\prime}$.

From the kinetic terms (5.15), the massless RR scalars are the uncharged scalars

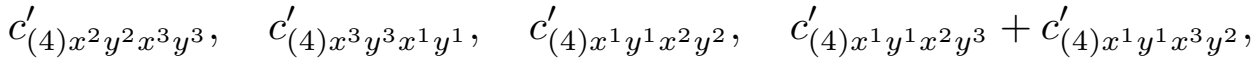

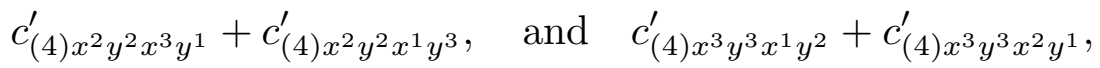

which are the axionic partners of $V_{1}^{\prime}, V_{2}^{\prime}, V_{3}^{\prime}, V_{(23)}^{\prime}, V_{(31)}^{\prime}$, and $V_{(12)}^{\prime}$, respectively, in $\mathcal{N}=1$ chiral multiplets.

The massive gauge bosons are those that couple to the other RR scalars. From (5.15), they lie in the 9D space spanned by

$$
(f / h) b_{(2) x^{i} \mu}^{\prime}+c_{(2) x^{i} \mu}^{\prime}, \quad b_{(2) y^{i} \mu}^{\prime}, \quad \text { and } \quad c_{(2) x^{i} \mu}^{\prime}+c_{(2) y^{i} \mu}^{\prime}, \quad i=1,2,3 .
$$

In terms of the complex gauge bosons $(5.16)$, this is the space spanned by

$$
\operatorname{Im}\left(\left(1 / \bar{\tau}^{\prime}\right) d_{z^{i} \mu}^{\prime}\right), \quad \operatorname{Re}\left(d_{\bar{z}^{i} \mu}^{\prime}\right), \quad \text { and } \quad \operatorname{Im}\left(d_{\bar{z}^{i} \mu}^{\prime}\right), \quad i=1,2,3
$$

The massless bosons are the linear combinations orthogonal to this space, where orthogonality is defined with respect to the metric appearing in the gauge kinetic terms. In the $\left(d_{z^{i} \mu}^{\prime}, d_{\bar{z}^{i} \mu}^{\prime}, \bar{d}_{z^{i} \mu}^{\prime}, \bar{d}_{\bar{z}^{i} \mu}^{\prime}\right)$ basis, the latter is proportional to

$$
\frac{1}{2}\left(\begin{array}{ll}
0 & 1 \\
1 & 0
\end{array}\right) \bar{d}^{d^{\prime}} \quad \otimes\left(\begin{array}{cc}
0 & g_{6}^{\prime z^{i} \bar{z}^{j}} \\
g_{6}^{\prime \bar{z}^{i} z^{j}} & 0
\end{array}\right) \bar{z}^{i}
$$


Therefore, the three massless gauge bosons are

$$
\operatorname{Re}\left(\left(1 / \bar{\tau}^{\prime}\right) d_{z^{i} \mu}^{\prime}\right) \propto(f / h)\left(b_{(2) x^{i} \mu}^{\prime}+b_{(2) y^{i} \mu}^{\prime}\right)+c_{(2) y^{i} \mu}^{\prime}, \quad i=1,2,3 .
$$

Finally, there are $6 \mathrm{M}$ moduli $\Phi_{I}$ and $M$ massless gauge bosons $A_{I \mu}$ from the D3 branes.

In summary, the massless fields are one graviton, $3+M$ vectors, and $6+6 M$ moduli. The moduli consist of

$$
\begin{gathered}
V_{1}^{\prime}, V_{2}^{\prime}, V_{3}^{\prime}, V_{(23)}^{\prime}, V_{(31)}^{\prime}, V_{(12)}^{\prime}, \\
6 \widetilde{c}_{(p)} \text { scalars, and } 6 M \text { D5 scalars. }
\end{gathered}
$$

These fields combine to form one $4 \mathrm{D} \mathcal{N}=1$ gravity multiplet, $3+3 M$ chiral multiplets, and $3+M$ vector multiplets.

From the supersymmetry-breaking mass spectra given in Ref. [17], this number of massless moduli and gauge bosons corresponds to $\mathcal{N}=4 \rightarrow \mathcal{N}=1$ with $m_{1}=m_{2}=m_{3}$ for the three massive gravitini. There are $3+3 M$ massless chiral multiplets and $3+M$ massless vector multiplets. The metric on moduli space is discussed in App. G. Since the complex structure modulus for each $T^{2}$ factor satisfies $\operatorname{Im} \tau^{\prime}>0$, the $6 \mathrm{D}$ spinor $\chi^{\prime}$ generating the supersymmetries can be written as the product of three $2 \mathrm{D}$ spinors, each of definite chirality along a $T^{2}$ (cf. the discussion at the end of Sec. 8.1).

\subsection{T-Duality Map}

If we return from the notation of Sec. 8.2 to the conventional labeling of coordinates via $(8.16)$, then it is straightforward to show that the metric $(8.28)$ can be written as a flat $T^{2}$ fibration (5.18), with

$$
\begin{aligned}
V_{\text {fib }}^{\prime}= & V_{1}^{\prime}, \\
a^{\prime 4}= & V_{1}^{\prime-1}\left(V_{(12)}^{\prime} d x^{6}+V_{(31)}^{\prime} d x^{8}\right) \\
a^{\prime 5}= & V_{1}^{\prime-1}\left(V_{(12)}^{\prime} d x^{7}+V_{(31)}^{\prime} d x^{9}\right) \\
d s_{\mathcal{B}}^{2}= & \frac{1}{\left|\operatorname{Im} \tau^{\prime}\right|}\left(\left(V_{2}^{\prime}-\left(V_{(12)}^{\prime}\right)^{2} / V_{1}\right) d z^{2} d \bar{z}^{2}+\left(V_{3}^{\prime}-\left(V_{(31)}^{\prime}\right)^{2} / V_{1}\right) d z^{3} d \bar{z}^{3}\right. \\
& \left.\quad+\left(V_{23}^{\prime}-V_{(12)}^{\prime} V_{(31)}^{\prime} / V_{1}\right)\left(d z^{2} d \bar{z}^{3}+d z^{3} d \bar{z}^{2}\right)\right) .
\end{aligned}
$$

The base metrics (8.8) and (8.34d) agree, provided that we make the identifications

$$
\begin{gathered}
V_{2}=V_{2}^{\prime}-\left(V_{(12)}^{\prime}\right)^{2} / V_{1}^{\prime}, \quad V_{3}=V_{3}^{\prime}-\left(V_{(31)}^{\prime}\right)^{2} / V_{1}^{\prime}, \\
V_{(23)}=V_{(23)}^{\prime}-V_{(12)}^{\prime} V_{(31)}^{\prime} / V_{1}^{\prime}, \quad \tau=\tau^{\prime} .
\end{gathered}
$$

All other quantities map exactly as described in Sec. 5.2. 


\section{Preview: $\mathcal{N}=2$ Calabi-Yau Duals without Flux 35}

So far, we have said nothing about $g_{s}$ or $\alpha^{\prime}$ corrections. We know that there are at least some such corrections, due to the following pathology in the leading $g_{s}, \alpha^{\prime}$ supergravity description of the orientifold. 36 As $x \rightarrow x^{\prime}$, the Green's function (3.31) behaves like a Green's function on $\mathbb{R}^{4}$ :

$$
G_{\mathcal{B}}\left(x, x^{\prime}\right) \sim \frac{1}{8 \pi^{2}}\left(\frac{1}{\left|x-x^{\prime}\right|^{2}}+c\right),
$$

where $c$ is a constant. So, near an O5 plane (with $Q_{\mathrm{O} 5}=-2$ ),

$$
Z(x) \sim 1-g_{s} \alpha^{\prime}\left(\frac{1}{\left|x-x_{\mathrm{O} 5}\right|^{2}}+c\right) .
$$

Consequently, as we let $x$ approach $x_{\mathrm{O} 5}$, we encounter a locus $Z=0$ where the metric is singular and $e^{\phi}=g_{s} Z^{-1 / 2}$ diverges. Beyond this locus, we enter the region $Z<0$, where the metric and $e^{\phi}$ are imaginary. A similar pathology afflicts the leading supergravity description of all orientifolds. On the other hand, orientifold planes are not singular objects in string theory. Therefore, the 10D effective field theory descending from string theory must get corrected in a way that resolves the pathology. In the $\mathcal{N}=2$ and $\mathcal{N}=4$ cases, it is easy to understand this resolution.

In the $\mathcal{N}=2$ example of Sec. 6.2 , there is an isometry in the $x^{9}$ direction (spontantanously broken by the positions of the eight $\mathrm{O} 5$ planes and $2 M$ D5 branes). If we T-dualize in this direction, we obtain an O6 orientifold, with O6 planes and D6 branes wrapping the $x^{4}, x^{5}, x^{9}$ directions. Since there was no NS flux before the T-duality, there is no new $S_{\left\{x^{9}\right\}}^{1}$ fibration introduced by the T-duality. The only flux after the T-duality is $F_{(2)}$, which is (the pullback to 6D of) a two-form in the $T_{\left\{x^{6}, x^{7}, x^{8}\right\}}^{3}$ directions. As noted in Ref. 迎, this IIA background lifts to a purely geometrical M theory background. The $F_{(2)}$ flux gives the fibration of the $\mathrm{M}$ theory circle $S_{\left\{x^{10}\right\}}^{1}$ over the IIA geometry. The dilaton gives the size of the fiber. Finally, the D6 branes and O6 planes, the only objects that are singular in the IIA supergravity description, lift locally to smooth Taub-NUT and smooth

35 I am grateful to P. Berglund, V. Braun, B. Florea, C. Johnson, R. Reinbacher, N. Warner, and C. Vafa for comments and suggestions related to this section. In particular, P. Berglund and $\mathrm{N}$. Warner first suggested studying the lift to $\mathrm{M}$ theory.

36 Here, leading order in $g_{s}$ means " $0+\frac{1}{2}$ " loop order, that is, tree level in the closed string sector plus tree level in the open string sector. 
Atiyah-Hitchin spaces times $\mathbb{R}^{6,1}$, respectively [78]. (The Atiyah-Hitchin space is the $\mathbb{Z}_{2}$ quotient of negative mass Taub-NUT space in which the region too close to the center has been smoothly excised. The excised region corresponds roughly to the unphysical region $Z<0$ of the orientifold.) Thus, the IIA orientifold lifts to

$$
\text { M theory on } Y_{7}, \quad \text { where } \quad Y_{7}=S_{\left\{x^{9}\right\}}^{1} \times \text { smooth } Y_{6} \text {. }
$$

There is no flux in $\mathrm{M}$ theory after the lift. The manifold $Y_{7}$ is truly a product, with no discrete identifications mixing the two factors. The orientifold $\mathbb{Z}_{2}$ operation lifts to a geometrical $\mathbb{Z}_{2}$ that inverts $x^{6}, x^{7}, x^{8}, x^{10}$. So, it goes into the definition of $Y_{6}$, but does not act on $S_{\left\{x^{9}\right\}}^{1}$. Since the M theory compactification preserves $4 \mathrm{D} \mathcal{N}=2$ supersymmetry, it follows that

$Y_{6}$ is a smooth Calabi-Yau threefold.

Compactifying on $S_{\left\{x^{9}\right\}}^{1}$ then gives a standard $\mathcal{N}=2$ Calabi-Yau compactification of IIA. Compactifying on $S_{\left\{x^{10}\right\}}^{1} \subset Y_{6}$ (with $Y_{6}$ viewed as a fibration) gives the $\mathrm{O} 6$ orientifold. Alternatively, $\mathrm{F}$ theory on $Y_{6} \times T^{2}$ gives the original $\mathrm{O} 5$ orientifold of Sec. 6.2, provided that we identify the F theory torus with $S_{\left\{x^{10}\right\}}^{1} \subset Y_{6}$ times $S^{1} \subset T^{2}$. Since this F theory $T^{2}$ fibration has no global section, there is automatically $F_{(3)}$ flux in the IIB orientifold 79].

Since the geometry $\mathbb{R}^{3,1} \times S^{1} \times Y_{6}$, with no flux, solves the equations of motion of $11 \mathrm{D}$ supergravity, the only essential ingredient that is missing in the leading IIA description but present in the $\mathrm{M}$ theory dual description is the dependence of the $11 \mathrm{D}$ background on the $x^{10}$ direction. The 10D IIA supergravity theory is the dimensional reduction of 11D supergravity truncated to lowest Fourier modes along the $x^{10}$ circle. The full Kaluza-Klein reduction of $11 \mathrm{D}$ supergravity includes not only the IIA supergravity fields, but also the entire tower of their D0-charged massive cousins from the bound states of $N$ D0 branes, for all $N \in \mathbb{N}$. Away from the O6 planes, if $g_{s}$ is tuned to be small, the D0 bound states are heavy, and it is a good approximation to ignore the massive tower. By tuning $g_{s}$ smaller and smaller, this approximation becomes valid closer and closer to the O6 planes. However, $e^{\phi}$ always diverges at some locus near the O6 planes. As we approach this locus, the D0 bound states become massless and cannot be neglected.

It is natural to ask what class of Calabi-Yau threefolds arises from the duality just described. Immediately, we know at least one piece of topological data. In Sec. 6.2 we determined that there were $n_{V}=2+M$ vector multiplets and $n_{H}=3+M$ hypermultiplets 
in addition to the gravity multiplet in the massless spectrum. On the other hand, for Calabi-Yau compactifications of type IIA, $n_{V}=h^{1,1}$ and $n_{H}=h^{2,1}+1$. Therefore,

$$
h^{1,1}\left(Y_{6}\right)=h^{2,1}\left(Y_{6}\right)=2+M,
$$

where the possible values of $M$ are $M=0,4,8$, and 12 . In the case that $M=16$, there is $\mathcal{N}=4$ supersymmetry and $Y_{6}$ is $T_{\left\{x^{4}, x^{5}\right\}}^{2} \times K 3_{\left\{x^{6}, x^{7}, x^{8}, x^{1} 0\right\}}^{\text {[80]. }}$.

Beyond this, it is possible to determine the intersection numbers from the special Kähler geometry of the moduli space (6.72b). Furthermore, for each $M$, there is not one Calabi-Yau, but topologically distinct geometries distinguished by the integers $m, n$ of Sec. 6 such that $4 m n=16-M$ :

\begin{tabular}{|c|c|c|c|c|}
\hline$M$ & $\left(h^{1,1}, h^{2,1}\right)$ & \multicolumn{3}{|c|}{$(m, n)$} \\
\hline 0 & $(2,2)$ & $(4,1)$, & $(2,2)$, & $(1,4)$ \\
\hline 4 & $(6,6)$ & $(3,1)$, & $(1,3)$ & \\
\hline 8 & $(10,10)$ & $(2,1)$, & $(1,2)$ & \\
\hline 12 & $(14,14)$ & $(1,1)$ & & \\
\hline
\end{tabular}

The integer $n$ tell us about $H_{1}\left(Y_{6}, \mathbb{Z}\right)$, which is pure torsion (and subsequently about $\pi_{1}\left(Y_{6}\right)$, whose abelianization is $\left.H_{1}\left(Y_{6}, \mathbb{Z}\right)\right)$. In the dual O3 orientifold of Sec. 6.4, S-duality interchanges the integers $m$ and $n .37$ Therefore, compactification of type IIA on $Y_{6}(m, n)$ and $Y_{6}(n, m)$ gives the same low energy effective field theory. Since both compactifications are of type IIA, the relation between $Y_{6}(m, n)$ and $Y_{6}(n, m)$ is something different from Calabi-Yau mirror symmetry.

The details of this duality will appear in a separate paper [56]. There, we will derive the further topological data mentioned in the previous paragraph. In addition, from the standard identifications between 11D supergravity and 10D type IIA supergravity, we will obtain an approximate metric for $Y_{6}$, together with its corresponding closed Kähler form and closed $(3,0)$ form. 38

37 To be precise, $m$ and $n$ are interchanged under S-duality followed by the coordinate relabeling $\left(x^{8}, x^{9}\right)_{\text {new }}=\left(x^{9},-x^{8}\right)_{\text {old }}$.

38 The approximate metric is Calabi-Yau on a noncompact space that excludes the singular region $Z^{\prime \prime} \leq 0$, where $Z^{\prime \prime}$ is the warp factor of the $O 6$ orientifold. This metric is the lowest Fourier mode of the exact Calabi-Yau metric on the compact manifold $Y_{6}$, in a Fourier expansion along the $x^{10}$ direction. It can in principle be systematically extended to the exact Calabi-Yau metric by including all higher Fourier modes, that is, by solving the 6D Einstein equations order by order in an $x^{10}$ Fourier expansion. 
An added incentive for studying this duality is that it could help us to compute other $g_{s}$ and $\alpha^{\prime}$ corrections in the original orientifold. For corrections that map to worldsheet and D instantons in the Calabi-Yau duals, we can hope to take advantage of the existing results for Calabi-Yau compactifications and then map the results back to the orientifold.

\section{Conclusions and Outlook}

Type IIB orientifolds with wrapped O5 planes and internal flux yield a class of 4D vacua that remains largely unexplored compared to its O3 and O7 counterparts. We have studied the simplest such vacua here. We have seen that these vacua, in which O5 planes and D5 branes wrap the $T^{2}$ fiber over a $T^{4}$ base, provide a computable toy model for studying moduli stabilization in a torsionful compactification. The supersymmetry conditions can be expressed in terms of a complex three-form flux $\widetilde{G}_{(3)}$ just as for D3/D7 or heterotic compactifications, and in addition a complex one-form flux $G_{(1)}$. This was illustrated in Secs. 6-8 through five different examples preserving $4 \mathrm{D} \mathcal{N}=1$, 2 , and 3 supersymmetry. As a check, we have also seen that the results of each example agree with the T-dual analysis in the corresponding $T^{6} / \mathbb{Z}_{2}$ orientifold. By providing an intrinsic description in the $\mathrm{O} 5$ orientifold, we have generalized the results of Ref. [4, which relied solely on the T-duality map at a special locus in moduli space.

One might have hoped that by giving a description of the $\mathrm{O} 5$ orientifold not grounded in T-duality, we would have found new solutions that do not T-dualize to the $T^{6} / \mathbb{Z}_{2}$ O3 orientifold. However, as observed in Sec. 3.1, the condition for the T-duality to fail to exist is that $H_{(3) \alpha \beta m}$, with two fiber indices, is nonzero, and this is exactly the case in which the analysis of the O5 orientifold qualitatively changes: the pseudo-BPS conditions of Sec. 3.3 no longer hold; moreover, this component of flux can be thought of as a transversely varying NS $B$-field oriented parallel to the D5 brane worldvolumes, which gives rise to noncommutativity. It would be interesting to prove that $\mathcal{N}>1$ supersymmetry demands that $H_{(3) \alpha \beta m}=0$.

There are a number of other questions left unanswered by this paper. In Sec. 3.9, we stated the flux quantization conditions in the O5 orientifold, but offered no proof. It is a significant omission that we were not able to derive these conditions directly in the O5 orientifold. The quantization conditions agree with those of the dual O3 orientifold via 
Eqs. (5.31). However, it should be possible, and would be more satisfactory, to instead derive the conditions directly from the appropriate twisted K-theory analysis. 39

In our description of the massless spectra, we were more explicit than in Ref. [4] for the $\mathrm{O} 5$ orientifold or Ref. [11] for the dual O3 orientifold. As a result, one feature that we noticed is that in all examples, the partial supersymmetry breaking from $\mathcal{N}=4$ to $\mathcal{N}=1,2$, or 3 is of the "nongeneric" type in which the massive gravitini have the same mass. A natural question is whether this is required. Do there exist supersymmetric $T^{6} / \mathbb{Z}_{2}$ flux vacua, with quantized flux satisfying the Gauss's law constraint, in which the superHiggs mechanism gives different masses to the gravitini? Also, our analysis of the massless spectrum proceeded on a case by case basis. Is there there a more elegant way of computing the massless spectrum through the cohomology of a suitable operator?

Other than in the introduction, we mentioned $S U(3)$ torsion classes in this paper only in Sec. 6.3, where the criterion $\mathcal{W}_{1} \neq 0$ was used to prove that the almost complex structure of Ex. 3 was nonintegrable. We did not employ $S U(2)$ torsion classes at all. The reason is that the torsion classes simply were not essential for our purposes of analyzing the supersymmetry conditions and moduli stabilization in this particular class of O5 vacua. Nevertheless, in recent months there has been considerable effort devoted to developing a framework that can be used to describe the most general 4D Minkowski vacua of type IIA and IIB string theory. (In the case of noncompact internal geometry this is closely related to studying type II or $\mathrm{M}$ theory solutions with an $A d S_{5}$ factor [36].) The correct framework for this analysis is in terms of $S U(2)$ structures and torsion classes [41, 36, 42, For the example of Sec. 6.3 , in which $\widetilde{F}_{(3)}, F_{(1)}$, and $H_{(3)}$ are all nonzero, we were able to avoid talking about $S U(2)$ structures by instead working in terms of the right- $S U(3)$ structures, and the volume form on the fiber. However, this is the one example of the paper in which supersymmetry does not provide us with a single 6D spinor, but two linearly independent spinors $\chi_{R}$ and $\chi_{L}=\gamma_{\mathcal{B}} \chi_{R}$. The two spinors canonically determine an $S U(2)$ structure rather than an $S U(3)$ structure. Therefore, this example should provide a useful testing ground for the IIB supersymmetry conditions as formulated in Ref. [42] in terms of $S U(2)$ torsion classes. One just needs to compute the torsion classes corresponding to the example. (In the other examples, the $S U(2)$ torsion collapses to $S U(3)$ torsion.) Working in terms of these torsion classes might have the added benefit of proving that the almost complex structure is always nonintegrable for the subclass of O5 backgrounds of the type

39 I am indebted to G. Moore for a helpful discussion on flux quantization. 
discussed here when there is NS flux, a result that we proved only for the specific example of Sec. 6.3.

Finally, perhaps the most interesting direction to pursue is to make more precise the duality of Sec. 9 relating $\mathcal{N}=2$ orientifold vacua to standard type IIA Calabi-Yau vacua. We have described a number of computable features of the dual Calabi-Yau theefolds and are optimistic about the prospects for identifying these manifolds. As one application of the identification, note that although it is common to speak of connected webs of $\mathcal{N}=2$ string vacua, given a pair of $\mathcal{N}=2$ vacua, it is not necessarily known whether the moduli spaces of the two are connected. For the class of $\mathcal{N}=2$ Calabi-Yau vacua, what is known is that the subspace of smooth Calabi-Yau threefolds that are hypersurfaces in toric varieties is connected. This subspace has trivial fundamental group, and has been completely described by Kreuzer and Skarke, who tabulated all 473,800,776 reflexive polyhedra in four dimensions [81]. In at least the case that $(M, m, n)=(12,1,1)$ and $h^{1,1}=h^{2,1}=14$, the Calabi-Yau $Y_{6}$ of Sec. 9 is conceivably contained in this subspace, and the dual orientifold contained in the corresponding web of known connected $\mathcal{N}=2$ vacua. This intriguing duality between $\mathcal{N}=2$ orientifold vacua and standard type IIA Calabi-Yau vacua is currently under investigation [56].

\section{Acknowledgements}

It is a pleasure to thank Allan Adams, Per Berglund, Andreas Brandhuber, Ram Brustein, Richard Corrado, Jerome Gauntlett, Jaume Gomis, Fawad Hassan, Anton Kapustin, Albion Lawrence, Christian Römelsberger, and especially Andrew Frey for helpful discussions and useful references. In addition, I thank S. Kachru, P. Tripathy, and S. Trivedi for the enjoyable collaboration from which this investigation is a continuous outgrowth. Finally, I am grateful to Stockholm University, the Aspen Center for Physics, the Kavli Institute for Theoretical Physics, the University of Pennsylvania, and Harvard University for hospitality during the course of this work. This work was supported in part by the DOE under contract DE-FG03-92-ER40701 and by the National Science Foundation under Grant No. PHY99-07949. 


\section{Appendix A. Conventions}

The conventions for coordinate indices are as follows:

$M, N, \ldots \quad$ denote $10 \mathrm{D}$ spacetime indices in the range $0, \ldots, 9$,

$\mu, \nu, \ldots \quad$ denote $4 \mathrm{D}$ spacetime indices in the range $0, \ldots, 3$,

$a, b, \ldots \quad$ denote $6 \mathrm{D}$ internal indices in the range $4, \ldots, 9$,

$\alpha, \beta, \ldots \quad$ denote $T^{2}$ fiber indices in the range 4,5 ,

$m, n, \ldots \quad$ denote $4 \mathrm{D}$ base indices in the range $6, \ldots, 9$,

$i, j, \ldots$ and $\bar{\imath}, \bar{\jmath}, \ldots$ denote complex $6 \mathrm{D}$ internal indices in the range $1,2,3$.

When 10D tensors (such as $R_{M N}$ or the fluxes) are decomposed into $\mu, \alpha, m$ components, the decomposition is with respect to the basis $d x^{\mu}, \eta^{\alpha}, d x^{m}$, not $d x^{\mu}, d x^{\alpha}, d x^{m}$, with one exception. In the context of the O3 orientifold, underscored indices $\underline{\mu}, \underline{\alpha}, \underline{m}$ denote components in the basis $d x^{\mu}, d x^{\alpha}, d x^{m}$.

The squares and partially contracted squares of 10D tensors are defined by

$$
\begin{aligned}
A_{(p)}^{2} & =A_{(p) M_{1} \ldots M_{p}} A_{(p)} M_{1} \ldots M_{p}, \\
A_{(p) M N}^{2} & =A_{(p) M Q_{2} \ldots Q_{p}} A_{(p) N}{ }^{Q_{2} \ldots Q_{p}},
\end{aligned}
$$

with the metric (2.4) used for raising and lowering indices. This metric is also used to define the square of the fibration curvature,

$$
\mathcal{F}^{2}=g_{\alpha \beta} g^{m p} g^{n q} \mathcal{F}_{m n}^{\alpha} \mathcal{F}_{p q}^{\beta}
$$

We follow the differential geometry conventions of Nakahara [82] for normalization of differential forms, exterior differentiation, Hodge duality, and curvature. (In the terminology of Misner, Thorne, and Wheeler [83], we employ +++ sign conventions.) In particular, on the $6 \mathrm{D}$ internal manifold,

$$
\begin{aligned}
& \omega \wedge *_{6} \omega=\frac{1}{p !} \omega_{a_{1} \ldots a_{p}} \omega^{a_{1} \ldots a_{p}} \operatorname{Vol}_{6}, \\
& \frac{1}{3 !} J \wedge J \wedge J=\frac{i}{8} \Omega \wedge \bar{\Omega}=\operatorname{Vol}_{6} .
\end{aligned}
$$

Compared to earlier work 99.11, 4, the Hodge star operator maps the same forms to one another, but $\left(\mathrm{Vol}_{6}\right)_{\text {here }}$ is minus $\left(\mathrm{Vol}_{6}\right)_{\text {there }}$. Therefore, the two possible selfduality conditions on middle dimensional forms,

$$
\begin{array}{ll}
*_{6} \omega_{(3)}=+i \omega_{(3)} & \text { imaginary-selfduality (ISD), } \\
*_{6} \omega_{(3)}=-i \omega_{(3)} & \text { imaginary-antiselfduality (IASD), }
\end{array}
$$


are the same here as in the earlier work [9:11, 国.

Our normalization conventions for 6D Hermitian metrics in complex coordinates $z^{i}, \bar{z}^{j}$ follow by compatibility with the expression for the metric in arbitrary $6 \mathrm{D}$ coordinates $x^{a, b}$ :

$$
d s^{2}=g_{a b} d x^{a} d x^{b}=g_{i \bar{\jmath}} d z^{i} d \bar{z}^{j}+g_{\bar{\imath} j} d \bar{z}^{i} d z^{j}=2 g_{i \bar{\jmath}} d z^{i} d z^{\bar{j}} .
$$

Note the factor of 2 on the RHS of the last equality.

The notation $\omega_{(p)}=\omega_{(p)}^{0}+\omega_{(p)}^{1}+\omega_{(p)}^{2}$ is defined in Sec. 3.2, and denotes the decomposition of an internal $6 \mathrm{D} p$-form $\omega_{(p)}$ into components $\omega_{(p)}^{i}$ of rank $i$ on the $T^{2}$ fiber.

Slashes denote contraction of tensors with 10D Dirac matrices,

$$
A_{(p) M_{1} \ldots M_{k}}=\frac{1}{(p-k) !} A_{(p) M_{1} \ldots M_{k}} \Gamma^{M_{k+1}} \ldots \Gamma^{M_{p}} .
$$

Antisymmetrized products of Dirac matrices are

$$
\begin{aligned}
\Gamma^{M_{1} \ldots M_{n}} & =\Gamma^{\left[M_{1}\right.} \Gamma^{M_{2}} \ldots \Gamma^{\left.M_{n}\right]}, \\
\gamma^{a_{1} \ldots a_{n}} & =\gamma^{\left[a_{1}\right.} \gamma^{a_{2}} \ldots \gamma^{\left.a_{n}\right]} .
\end{aligned}
$$

The antisymmetrized product of all ten $\Gamma^{M}$ gives the 10D chirality operator,

$$
\Gamma^{(10)}=-g^{1 / 2} \Gamma^{0123456789}
$$

where $g_{M N}$ is the 10D metric (2.4). Uppercase Dirac matrices satisfy

$$
\left\{\Gamma_{M}, \Gamma_{N}\right\}=2 g_{M N}
$$

Lowercase Dirac matrices satisfy

$$
\left\{\gamma_{\mu}, \gamma_{\nu}\right\}=2 \eta_{\mu \nu}, \quad\left\{\gamma_{a}, \gamma_{b}\right\}=2 g_{a b}
$$

where $\eta=\operatorname{diag}(-1,1,1,1)$ and $g_{a b}$ is the $6 \mathrm{D}$ internal metric (2.7).

The relation between uppercase and lowercase Dirac matrices is

$$
\Gamma_{\mu}=Z^{-1 / 4} \gamma_{\mu} \otimes 1, \quad \Gamma_{a}=Z^{-1 / 4} \gamma^{(4)} \otimes \gamma_{a}
$$

where

$$
\gamma^{(4)}=i \gamma^{0123}
$$


Here, aside from the overall warp factor (cf. Eq. (2.8)), we follow Candeles, Horowitz, Strominger, and Witten [5]. We also follow the latter in employing a Majorana representation. The $\Gamma_{M}, \gamma_{\mu}$ are real and Hermitian, except for $\Gamma_{0}, \gamma_{0}$, which are real and antiHermitian. The $\gamma_{a}$ are imaginary and Hermitian.

In addition, we define

$$
\begin{aligned}
\Gamma^{(4)} & =i Z^{-1} \Gamma^{0123}, \\
\Gamma^{(6)} & =i Z^{1 / 2} g_{6}^{1 / 2} \Gamma^{456789}, \\
\Gamma_{\text {fib }} & =i Z^{-1 / 2}\left(g^{\left(T_{\text {fib }}^{2}\right)}\right)^{1 / 2} \Gamma^{45}, \\
\Gamma_{\mathcal{B}} & =Z g_{\mathcal{B}}^{1 / 2} \Gamma^{6789},
\end{aligned}
$$

and similarly,

$$
\begin{aligned}
\gamma^{(6)} & =i g_{6}^{1 / 2} \gamma^{456789} \\
\gamma_{\mathrm{fib}} & =i\left(g^{\left(T_{\mathrm{fib}}^{2}\right)}\right)^{1 / 2} \gamma^{45} \\
\gamma_{\mathcal{B}} & =Z^{2} g_{\mathcal{B}}^{1 / 2} \gamma^{6789}
\end{aligned}
$$

Since $\left(\gamma^{(4)}\right)^{2}=1$, we then have the relations

$$
\begin{aligned}
& \Gamma^{(4)}=\gamma^{(4)} \otimes 1, \quad \Gamma_{\text {fib }}=1 \otimes \gamma_{\text {fib }}, \quad \text { and } \quad \Gamma_{\mathcal{B}}=1 \otimes \gamma_{\mathcal{B}}, \\
& \Gamma^{(6)}=\Gamma_{\text {fib }} \Gamma_{\mathcal{B}}=1 \otimes \gamma^{(6)}, \quad \Gamma^{(10)}=\Gamma^{(4)} \Gamma^{(6)}=\gamma^{(4)} \otimes \gamma^{(6)},
\end{aligned}
$$

as well as

$$
\Gamma^{a_{1} \ldots a_{2 n}}=Z^{n / 2} 1 \otimes \gamma^{a_{1} \ldots a_{2 n}}, \quad \Gamma^{a_{1} \ldots a_{2 n+1}}=Z^{(2 n+1) / 4} \gamma^{(4)} \otimes \gamma^{a_{1} \ldots a_{2 n}} .
$$

In our representation conventions, $\gamma^{(4)}, \gamma^{(6)}$, and $\gamma_{\text {fib }}$ are imaginary and Hermitian, while $\gamma_{\mathcal{B}}$ is real and Hermitian. Thus, complex conjugation $\chi \rightarrow \chi^{*}$ of a $6 \mathrm{D}$ internal spinor reverses its $\gamma^{(6)}$ and $\gamma_{\text {fib }}$ chirality, but leaves its $\gamma_{\mathcal{B}}$ chirality unchanged. Complex conjugation $u \rightarrow u^{*}$ of a $4 \mathrm{D}$ spacetime spinor $u$ reverses its $\gamma^{(4)}$ chirality.

We can further decompose the lowercase Dirac matrices as

$$
\gamma_{\alpha}=\widetilde{\gamma}_{\alpha} \otimes 1, \quad \gamma_{m}=Z^{1 / 2} \widetilde{\gamma}_{\text {fib }} \otimes \widetilde{\gamma}_{m},
$$

where

$$
\widetilde{\gamma}_{\mathrm{fib}}=i\left(g^{\left(T_{\mathrm{fib}}^{2}\right)}\right)^{1 / 2} \widetilde{\gamma}^{45}, \quad \widetilde{\gamma}_{\mathcal{B}}=g_{\mathcal{B}}^{1 / 2} \widetilde{\gamma}^{6789}
$$

and

$$
\left\{\widetilde{\gamma}_{\alpha}, \widetilde{\gamma}_{\beta}\right\}=2 g_{\alpha \beta}^{\left(T_{\text {fib }}^{2}\right)}, \quad\left\{\widetilde{\gamma}_{m}, \widetilde{\gamma}_{n}\right\}=2 g_{\mathcal{B} m n}
$$


Since $\gamma_{\mathrm{fib}}{ }^{2}=1$, we then have the relations

$$
\gamma_{\mathrm{fib}}=\widetilde{\gamma}_{\mathrm{fib}} \otimes 1, \quad \gamma_{\mathcal{B}}=1 \otimes \widetilde{\gamma}_{\mathcal{B}}
$$

and finally

$$
\gamma_{\alpha}{ }^{\beta}=\widetilde{\gamma}_{\alpha}^{\beta} \otimes 1, \quad \gamma_{m}^{n}=1 \otimes \widetilde{\gamma}_{m}^{n}, \quad \gamma_{\alpha m n}=Z \widetilde{\gamma}_{\alpha} \otimes \widetilde{\gamma}_{m n}
$$

which are needed in Sec. 4.2 .

The convention for \pm subscripts on $6 \mathrm{D}, 4 \mathrm{D}$, and $2 \mathrm{D}$ spinors is that the subscripts always indicate the fiber chirality and/or minus the base chirality. Therefore, the only $6 \mathrm{D}$ spinors that we refer to directly are of negative $\gamma^{(6)}$ chirality.

\section{Appendix B. Coordinate Identifications}

The fiber coordinates are periodically identified via

$$
x^{\alpha} \cong x^{\alpha}+1 \text { at fixed }\left\{x^{p}, x^{\beta}\right\}_{\beta \neq \alpha}
$$

In the case that the base $\mathcal{B}$ is a torus, Eq. (2.5) implies that

$$
\mathcal{A}^{\alpha}=\frac{1}{2} \mathcal{F}_{m n}^{\alpha} x^{m} d x^{n}, \quad \mathcal{F}_{m n}^{\alpha}=\text { const } \in \mathbb{Z}
$$

up to a fiber coordinate redefinition

$$
x_{\text {new }}^{\alpha}=x^{\alpha}+\Lambda^{\alpha}, \quad \mathcal{A}_{\text {new }}^{\alpha}=\mathcal{A}^{\alpha}-d \Lambda^{\alpha}, \quad \text { where } \Lambda^{\alpha}=\Lambda^{\alpha}\left(\left\{x^{m}\right\}\right) .
$$

In the gauge $(\mathbb{B} .2)$, the base coordinates have a periodic identification that also acts on the fiber coordinates:

$$
\left(x^{m}, x^{\alpha}\right) \equiv\left(x^{m}+1, x^{\alpha}-\frac{1}{2} \mathcal{F}_{m n}^{\alpha} x^{n}\right) \text { at fixed }\left\{x^{p}, x^{\beta}\right\}_{(p, \beta) \neq(m, \alpha)} .
$$

This twisted identification is the unique coordinate identification that both projects to $x^{m} \equiv x^{m}+1$ on the base and is compatible with

$$
\eta^{\alpha}=\left.\left(d x^{\alpha}+\mathcal{A}^{\alpha}\right)\right|_{x^{m}}=\left.\left(d x^{\alpha}+\mathcal{A}^{\alpha}\right)\right|_{x^{m}+1} \text { at fixed }\left\{x^{p}, x^{\beta}\right\}_{(p, \beta) \neq(n, \alpha)} \text {. }
$$

Other gauge choices lead to similar coordinate identifications. 


\section{Appendix C. Derivation of Pseudo-BPS Constraints}

Let

$$
\begin{aligned}
& \Delta_{1}=-g^{\mu \nu}\left(R_{\mu \nu}+2 \nabla_{\mu} \nabla_{\nu} \phi\right), \\
& \Delta_{2}=-g^{\alpha \beta}\left(R_{\alpha \beta}+2 \nabla_{\alpha} \nabla_{\beta} \phi\right)+\frac{1}{4} \mathcal{F}^{2}, \\
& \Delta_{3}=-\nabla^{2} \phi+2(\nabla \phi)^{2},
\end{aligned}
$$

and define

$$
\Delta=\Delta_{1}+\Delta_{2}+\Delta_{3}
$$

The warp factor $Z$ and dilaton $\phi$ are assumed to depend only on the base coordinates. For such a function $f$, we have

$$
\begin{aligned}
\nabla_{\mu} \nabla_{\nu} f & =Z^{-1} g_{\mathcal{B}}^{m m^{\prime}}(\log Z),_{m^{\prime}} \eta_{\mu \nu} \\
\nabla_{\alpha} \nabla_{\beta} f & =Z^{-1} g_{\mathcal{B}}^{m m^{\prime}}(\log Z),_{m^{\prime}} \delta_{\alpha \beta} \\
\nabla_{m} \nabla_{n} f & =\nabla_{\mathcal{B} m} \nabla_{\mathcal{B} n}-\frac{1}{4} Z^{-1}\left(-Z,_{k^{\prime}} g_{\mathcal{B}}^{k^{\prime} k} g_{\mathcal{B} m n}+Z,_{m} \delta_{n}^{k}+Z,_{n} \delta_{m}^{k}\right) f,_{k} .
\end{aligned}
$$

Using Eqs. (3.2) and (C.3), it is straightfoward to show that

$$
\begin{aligned}
\nabla_{\mathcal{B}} \cdot\left(\left(Z^{-1} e^{-2 \phi}\right) \nabla_{\mathcal{B}}\left(Z^{-3 / 2} e^{-\phi}\right)\right) & =Z^{1 / 2}\left(Z^{-1} e^{-2 \phi}\right)\left(Z^{-3 / 2} e^{-\phi}\right) \Delta \\
+ & \left(Z^{-1} e^{-2 \phi}\right)\left(Z^{3 / 2} e^{\phi}\right)\left(\nabla_{\mathcal{B}}\left(Z^{-3 / 2} e^{-\phi}\right)\right)^{2},
\end{aligned}
$$

where contractions are performed using base metric $g_{\mathcal{B} m n}$. On the other hand, from Eqs. (3.1) and (3.4), we have 40

$$
\begin{aligned}
\Delta_{1} & =e^{2 \phi}\left(F_{(1)}^{2}+\frac{1}{3 !} \widetilde{F}_{(3)}^{2}+\frac{1}{2} \frac{1}{5 !} \widetilde{F}_{(5)}^{2}\right)+(2 \pi)^{2} \alpha^{\prime} e^{\phi} \sum Q_{i} \frac{\delta^{4}\left(x-x_{i}\right)}{Z \sqrt{g_{\mathcal{B}}}}, \\
\Delta_{2}-\frac{1}{2} \Delta_{1} & =-\frac{1}{2} e^{2 \phi}\left(\frac{1}{3 !}\left(\widetilde{F}_{(3)}^{1}\right)^{2}+\frac{1}{5 !}\left(\widetilde{F}_{(5)}^{1}\right)^{2}+\frac{2}{5 !}\left(\widetilde{F}_{(5)}^{2}\right)^{2}\right)+\frac{1}{4} \mathcal{F}^{2} \\
-\Delta_{3} & =e^{2 \phi}\left(F_{(1)}{ }^{2}+\frac{1}{2} \frac{1}{3 !} \widetilde{F}_{(3)}{ }^{2}-\frac{1}{2} \frac{1}{3 !} H_{(3)}{ }^{2}\right)+\frac{1}{2}(2 \pi)^{2} \alpha^{\prime} e^{\phi} \sum Q_{i} \frac{\delta^{4}\left(x-x_{i}\right)}{Z \sqrt{g_{\mathcal{B}}}} .
\end{aligned}
$$

Therefore,

$$
\begin{aligned}
\Delta= & \frac{1}{2} e^{2 \phi}\left(F_{(1)}^{2}+\frac{2}{3 !}\left(\widetilde{F}_{(3)}^{0}\right)^{2}+\frac{1}{3 !}\left(\widetilde{F}_{(3)}^{1}\right)^{2}+\frac{2}{5 !}\left(\widetilde{F}_{(5)}^{1}\right)^{2}+\frac{1}{5 !}\left(\widetilde{F}_{(5)}^{2}\right)^{2}\right) \\
& +\frac{1}{2} \frac{1}{3 !}\left(H_{(3)}^{0}\right)^{2}+\frac{1}{2} \frac{1}{2 !} \mathcal{F}^{2}+(2 \pi)^{2} \alpha^{\prime} e^{\phi} \sum Q_{i} \frac{\delta^{4}\left(x-x_{i}\right)}{Z \sqrt{g_{\mathcal{B}}}} .
\end{aligned}
$$

40 Here, $\widetilde{F}_{(5)}=(1-*) \widetilde{F}_{(5)}^{\text {int }}$, where $\widetilde{F}_{(5)}^{\text {int }}=\sum \widetilde{F}_{(5)}^{i}$ denotes the purely internal part of $\widetilde{F}_{(5)}$, with no $4 \mathrm{D}$ spacetime indices. Note that this implies a relative factor of 2 in $\frac{1}{2} \frac{1}{5 !}\left(\widetilde{F}^{(5)}\right)^{2}=\frac{1}{5 !}\left(\widetilde{F}_{(5)}^{\text {int }}\right)^{2}$. 
By substituting Eq. (C.6) into Eq. (C.4) and adding to the result $Z^{-3} e^{-2 \phi}$ times Eq. (3.14a), we obtain the desired pseudo-BPS condition Eq. (3.18).

In writing Eqs. (C.5) and (C.6), we have assumed that $H_{(3)}^{2}=0$. If this is not the case, then there is an additional term

$$
\delta\left(\Delta_{2}-\frac{1}{2} \Delta_{1}\right)=-e^{2 \phi} \frac{1}{3 !}\left(\widetilde{F}_{(3)}^{2}\right)^{2}-\frac{1}{5 !}\left(H_{(3)}^{2}\right)^{2}
$$

on the RHS of the second equation (C.5), and additional terms

$$
\delta \Delta=\frac{3}{2} e^{2 \phi} \frac{1}{3 !}\left(\widetilde{F}_{(3)}^{2}\right)^{2}-\frac{1}{2} \frac{1}{3 !}\left(H_{(3)}^{2}\right)^{2}
$$

on the RHS of Eq. (C.6).

Here $\widetilde{F}_{(3)}^{2}=-C_{0} H_{(3)}^{2}$, where $C_{(0)}$ is a local section of a $U(1)$ bundle (such that $\left.F_{(1)}=d C_{(0)}\right)$ and cannot be written as a single-valued function. Since $\widetilde{F}_{(3)}^{2}$ and $H_{(3)}^{2}$ are gauge-invariant globally-defined three-forms, this implies that $F_{(1)}$ and $H_{(3)}^{2}$ cannot simultaneously be nonzero. Therefore, $F_{(3)}^{2}=0$. However, even when $F_{(1)}=0$ and $H_{(3)}^{2} \neq 0$, the second term in Eq. (C.8) still presents a problem; since it is negative, we obtain an additional negative term on the RHS of Eq. (3.18), and can no longer deduce the Hodge duality relations (3.20). Therefore, we restrict to the case $H_{(3)}^{2}=0$, in which we know how to proceed.

\section{Appendix D. IIB Fermion Variations}

The string frame fermion variations to linear order in the spinors are

$$
\begin{aligned}
\delta_{L, R} \lambda_{L, R} & =\frac{1}{2}\left(\not \partial \phi \mp \frac{1}{2} \not H_{(3)}\right) \epsilon_{L, R}, \\
\delta_{R, L} \lambda_{L, R} & =\frac{1}{2} e^{\phi}\left( \pm \mathscr{F}_{(1)}+\frac{1}{2} \widetilde{F}_{(3)}\right) \epsilon_{R, L}, \\
\delta_{L, R} \psi_{L, R M} & =\left(\nabla_{M} \mp \frac{1}{4} \mathscr{H}_{(3) M}\right) \epsilon_{L, R}, \\
\delta_{R, L} \psi_{L, R M} & =\frac{1}{8} e^{\phi}\left(\mp \mathscr{F}_{(1)}-\widetilde{F}_{(3)} \mp \frac{1}{2} \widetilde{F}_{(5)}\right) \Gamma_{M} \epsilon_{R, L} .
\end{aligned}
$$

Here, subscripts to the left (right) of a comma are correlated with upper (lower) sign choices. The subscripts $L$ and $R$ indicate whether the spin content of a fermion comes from the left-moving or right-moving sector of the worldsheet conformal field theory. In 
this appendix, we follow Hassan [75], except for a sign difference in the chirality of all of the $10 \mathrm{D}$ spinors, due to opposite conventions for $\Gamma^{(10)}$. We take

$$
\Gamma^{(10)} \epsilon_{L, R}=-\epsilon_{L, R}, \quad \Gamma^{(10)} \lambda=+\lambda, \quad \text { and } \quad \Gamma^{(10)} \psi_{L, R M}=-\psi_{L, R M},
$$

where $\Gamma^{(10)}$ is given by Eq. (A.8). Slashes denote contraction with Dirac matrices as defined in Eq. (A.6). Also,

$$
\nabla_{M} \epsilon_{L, R}=\left(\partial_{M}+\frac{1}{4} w_{M a b} \Gamma^{a b}\right) \epsilon_{L, R}
$$

where $w_{M a b}$ is the spin connection, and

$$
\widetilde{F}_{(n) M_{1} \ldots M_{n}}=n \partial_{\left[M_{1}\right.} C_{\left.(n-1) M_{2} \ldots M_{n}\right]}-\frac{n !}{3 !(n-3) !} H_{(3)\left[M_{1} M_{2} M_{3}\right.} C_{\left.(n-3) M_{4} \ldots M_{n}\right]} .
$$

The last equation means that

$$
\begin{aligned}
\widetilde{F}_{(n)} & =F_{(n)}-H_{(3)} \wedge C_{(n-3)}, \\
d \widetilde{F}_{(n)} & =H_{(3)} \wedge F_{(n-2)}+\text { local sources. }
\end{aligned}
$$

By defining the complex quantities

$$
\delta=\delta_{L}+\delta_{R}, \quad \epsilon=\epsilon_{L}+i \epsilon_{R}, \quad \lambda=\lambda_{L}+i \lambda_{R}, \quad \text { and } \quad \psi_{M}=\left(\psi_{L}+i \psi_{R}\right)_{M},
$$

we can equivalently write the fermion variations as

$$
\begin{aligned}
& \delta \lambda=\frac{1}{2}\left(i \not \partial \phi-e^{\phi} \mathscr{F}_{(1)}\right) \epsilon^{*}+\frac{1}{4}\left(e^{\phi} \widetilde{F}_{(3)}-i H_{(3)}\right) \epsilon, \\
& \delta \psi_{M}=\left(\nabla_{M}+\frac{i}{8} e^{\phi}\left(\mathscr{F}_{(1)} \Gamma_{M}+\frac{1}{2} \mathscr{F}_{(5)} \Gamma_{M}\right)\right) \epsilon-\frac{i}{8}\left(e^{\phi} \widetilde{F}_{(3)} \Gamma_{M}-2 i \mathbb{H}_{(3) M}\right) \epsilon^{*} .
\end{aligned}
$$

In terms of the axion-dilaton and complex flux, 41

$$
\begin{aligned}
\tau_{\text {dil }} & =C_{(0)}+i e^{-\phi}, \\
G_{(3)} & =\widetilde{F}_{(3)}-i e^{-\phi} H_{(3)}=F_{(3)}-\tau H_{(3)},
\end{aligned}
$$

41 Here, we have given the axion-dilaton for type IIB supergravity with no orientifold projection. This is also the axion-dilaton for orientifolds with O3 or O7 planes and Becker-type spinor constraints. However, as discussed in Sec. 4.2, a different quantity plays the role of the axiondilaton for $\mathrm{O} 5$ orientifolds, since the zero mode of $C_{(0)}$ is projected out. For the type I orientifold with O9 planes, $\tau_{\text {dil }}=a+i e^{-\phi}$, where $a$ is the scalar dual to $C_{(2) \mu \nu}$. 
this becomes

$$
\begin{aligned}
& e^{-\phi} \delta \lambda=-\frac{1}{2}\left(\not \partial \tau_{\mathrm{dil}}\right) \epsilon^{*}+\frac{1}{4} \phi_{(3)} \epsilon \\
& e^{-\phi}\left(\delta \psi_{M}-\frac{i}{4} \Gamma_{M} \delta \lambda^{*}\right)=\left(e^{-\phi}\left(\nabla_{M}-\frac{1}{8} \Gamma_{M} \not \partial \phi\right)+\frac{i}{4} F_{(1) M}+\frac{i}{16} \widetilde{F}_{(5)} \Gamma_{M}\right) \epsilon \\
&-i\left(\frac{1}{8} \phi_{(3)} \Gamma_{M}+\frac{1}{16} \Gamma_{M} \phi_{(3)}\right) \epsilon^{*}
\end{aligned}
$$

Here, we have assumed a real representation of the Dirac matrices. We have also used the relation

$$
\left\{\text { HI }_{(3)}, \Gamma_{M}\right\}=2 \text { H }_{(3) M}
$$

in deriving $(\overline{D .9})$.

When expressed in terms of the complex quantities $\tau_{\text {dil }}$ and $G_{(3)}$, the equations simplify in Einstein frame. Using a prime to indicate the latter, the relations between string frame and Einstein frame are

$$
\begin{aligned}
& g_{M N}^{\prime}=e^{-\phi / 2} g_{M N}, \quad \Gamma_{M}^{\prime}=e^{-\phi / 4} \Gamma_{M}, \quad \nabla_{M}^{\prime}=\nabla_{M}-\frac{1}{8} \Gamma_{M}^{N} \partial_{N} \phi, \\
& \epsilon^{\prime}=e^{\phi / 8} \epsilon, \quad \lambda^{\prime}=e^{\phi / 8} \lambda, \quad \psi_{M}^{\prime}=e^{-\phi / 8}\left(\psi_{M}-\frac{i}{4} \Gamma_{M} \lambda^{*}\right) .
\end{aligned}
$$

Therefore, the Einstein frame analog of Eqs. (D.9) is

$$
\begin{aligned}
\delta \lambda^{\prime} & =-\frac{1}{2} e^{\phi}\left(\not \partial^{\prime} \tau_{\mathrm{dil}}\right) \epsilon^{*}+\frac{1}{4} e^{\phi / 2} \phi_{T_{(3)}}^{\prime} \epsilon^{\prime}, \\
\delta \psi_{M}^{\prime} & =\left(\nabla_{M}^{\prime}+\frac{i}{4} e^{\phi} F_{(1) M}+\frac{i}{16}{\widetilde{F^{\prime}}}_{(5)}^{\prime} \Gamma_{M}^{\prime}\right) \epsilon^{\prime}-i e^{\phi / 2}\left(\frac{1}{8} \phi_{(3)}^{\prime} \Gamma_{M}^{\prime}+\frac{1}{16} \Gamma_{M}^{\prime}{\phi t_{(3)}^{\prime}}_{(3)} \epsilon^{\prime *},\right.
\end{aligned}
$$

where a slash and prime denotes the analog of Eq. (A.6) with $\Gamma_{M} \rightarrow \Gamma_{M}^{\prime}$.

Eqs. (D.12) give the Einstein frame fermion variations in the usual string theory conventions, in which $F_{(p)}, H_{(3)}$, and $G_{(3)}$ are the same in string frame and Einstein frame. In the supergravity literature, the quantity $G_{(3)}$ is given a slightly different definition. The relation between that definition and the one given in Eqs. (D.8) has been discussed elsewhere [6,75].

\section{Appendix E. The Meaning of $W_{\mathrm{GVW}}$ in the O3 Orientifold with Internal $T^{6}$}

As mentioned in Sec. 5.1, one subtlety in our description of metric moduli stabilization for the $\mathrm{O} 3$ orientifold based on internal $T^{6}$ is that not all of the (18 real) $\tau^{i}{ }_{j}$ and (9 real) $g_{i \bar{\jmath}}$ correspond to the (21 real) physical metric moduli. 
In contrast to the case for a proper Calabi-Yau threefold, there is not a one-to-one correspondence

$$
\begin{aligned}
& \delta \tau^{i}{ }_{j} D_{\tau^{i}{ }_{j}} \Omega=\omega^{(1,2)} \quad(\text { sum on } i, j) \\
& \delta g_{k l}=\Omega_{(k \mid}{ }_{k} \bar{l} \omega_{\bar{k} \bar{l} \mid l)}^{(1,2)}
\end{aligned}
$$

between deformations of complex structure $\left((1,2)\right.$ forms or $T^{(0,1)}$-valued $(1,0)$ forms $)$ and deformations of $g_{i j}$. The reason is that for a manifold such as $T^{6}$ with nontrivial $H^{(0,1)}$, there exist nonprimitive $(1,2)$ forms

$$
\omega^{(1,2)}=J \wedge \omega^{(0,1)}
$$

that generate vanishing metric deformations. For a $T^{6}$, the three such forms are $J \wedge d \bar{z}^{i}$. Correspondingly, there are three complex unphysical degrees of freedom in $\tau^{i}{ }_{j}$ that do not correspond to metric degrees of freedom.

This can be understood in the supergravity theory as follows. In the absence of flux, the theory has $\mathcal{N}=4$ supersymmetry. The fluxes break this to $\mathcal{N}<4$ supersymmetry, but the breaking should still be described within the formalism of $\mathcal{N}=4$ gauged supergravity. Unlike global $\mathcal{N}=4$ supersymmetry, $\mathcal{N} \geq 1$ supergravity cannot be cleanly described in $\mathcal{N}=1$ language, due to the lack of a simple off-shell formulation for $\mathcal{N}=1$ gravitino multiplets with a single auxiliary field analogous to the $D$ or $F$ fields for vector or chiral multiplets. 12 Nevertheless, upon choosing an $\mathcal{N}=1$ subalgebra of $\mathcal{N}=4$, one can still organize all fields into $\mathcal{N}=1$ multiplets and decompose the bosonic parts of all supersymmetry variations into $D$-terms, $F$-terms, and "other-terms." In this sense, we can still define an $\mathcal{N}=1$ superpotential, whose variation gives the $F$-terms. However, this superpotential is part of a family of superpotentials parametrized by a choice of the $\mathcal{N}=1$ subalgebra. Equivalently, it is parametrized by a choice of a point in the space

$$
Y=\frac{S U(4)_{\mathcal{R}}}{U(1)_{\mathcal{R}} \times S U(3)}, \quad \operatorname{dim}_{\mathbb{C}} Y=3
$$

of embeddings of the $U(1) \mathcal{R}$-symmetry of $\mathcal{N}=1$ in the $S U(4) \mathcal{R}$-symmetry of $\mathcal{N}=4$. Here, the $S U(3)$ is the commutant of $U(1)_{\mathcal{R}}$ in $S U(4)_{\mathcal{R}}$. For $T^{6}, W_{\mathrm{GVW}}$ is exactly this type of family of superpotentials, parametrized by the three complex unphysical degrees

42 Here, we have in mind an off-shell multiplet whose on-shell dynamical degrees of freedom are a spin $3 / 2$ field and a spin 1 field. For work on off-shell gravitino multiplets, see Ref. [84]. 
of freedom in $\tau^{i}{ }_{j}$. To obtain the $F$-terms, $W_{\mathrm{GVW}}$ should only be varied with respect to the physical degrees of freedom. The $F$-terms impose the conditions

$$
G_{(3)}=(1,2) \text {-nonprimitive }+(2,1)
$$

where the (1,2)-nonprimitive component is of the form (E.2).

On the other hand, in an operational sense, varying $W_{\mathrm{GVW}}$ with respect to $\tau_{\text {dil }}$ and all of the $\tau^{i}{ }_{j}$, including unphysical degrees of freedom, is a convenient thing to do: it is more easily implemented in practice than varying with respect to only physical deformations, and this seeming unphysical procedure imposes exactly the $(2,1)$ condition on $G_{(3)}$, which is still a subset of the supersymmetry conditions. The philosophy of Ref. [11] was simply to use $W_{\mathrm{GVW}}$ in this seemingly unphysical way, as a convenient tool for imposing the $(2,1)$ condition.

One disadvantage of this approach is that it is not always clear how many physical moduli there are among the redundent set of all $\tau^{i}{ }_{j}$ and $g_{i \bar{\jmath}}$ unfixed by the supersymmetry conditions. For the examples that we present, this is an issue only in the $\mathcal{N}=2$ case. (For our $\mathcal{N}=1$ and $\mathcal{N}=3$ examples, $\tau_{\text {dil }}$ and all complex structure moduli are fixed, and all of the unfixed Kähler moduli are physical.) We avoid the problem by choosing a noncanonical decomposition of unfixed metric moduli into Kähler and complex structure moduli in Sec. 6.4. The choice is natural from the point of view of the dual O5 orientifold of Sec. 6.2. The choice corresponds exactly to the choice of $\chi_{+}=0$ or $\chi_{-}=0$ in Sec 4.2, which leads to an integrable complex structure possessing a fiber $\oplus$ base decomposition as in Eq. (4.21).

\section{Appendix F. T-Duality Map for RR Potentials}

The T-duality map for the RR potentials $C_{(p)}$ is analogous to Eq. (5.28). The relations between components in the $\eta^{\alpha}, d x^{m}$ basis of the 05 orientifold and the $\eta^{\prime \alpha}, d x^{m}$ basis of the $\mathrm{O} 3$ orientifold are

$$
\begin{aligned}
C_{(p)}^{0} & =\frac{1}{2} \epsilon^{\alpha \beta} C_{(p+2) \alpha \beta}^{2} /\left((2 \pi)^{2} \alpha^{\prime}\right), \\
C_{(p)}^{1} & =-\epsilon^{\alpha \beta} C_{(p) \beta}^{\prime 1}, \\
C_{(p) \alpha \beta}^{2} & =-\epsilon^{\alpha \beta} C_{(p-2)}^{\prime 0}(2 \pi)^{2} \alpha^{\prime} .
\end{aligned}
$$

We assume that $H_{(3) \underline{\alpha \delta m}}^{\prime}=0$ so that the T-duality exists, but retain $F_{(3) \underline{m n r}}^{\prime}$. (The equations of motion ultimately require that $F_{(3) \underline{m n r}}^{\prime}=0$.) 
In the $\mathrm{O} 3$ orientifold, a choice of gauge for the potentials leading to the quantized flux (5.4) is

$$
\begin{aligned}
& B_{(2)}^{\prime \mathrm{bg}}=\frac{1}{2} H_{(3) \underline{\alpha n r}}^{\prime} x^{r} d x^{\alpha} \wedge d x^{n}+\frac{1}{3 !} H_{(3) \underline{m n r}}^{\prime} x^{r} d x^{m} \wedge d x^{n} \\
& C_{(2)}^{\prime \mathrm{bg}}=\frac{1}{2} F_{(3) \underline{\alpha \delta r}}^{\prime} x^{r} d x^{\alpha} \wedge d x^{\delta}+\frac{1}{2} F_{(3) \underline{\alpha n r}}^{\prime} x^{r} d x^{\alpha} \wedge d x^{n}+\frac{1}{3 !} F_{(3) \underline{m n r}}^{\prime} x^{r} d x^{m} \wedge d x^{n} .
\end{aligned}
$$

In the $\eta^{\alpha}, d x^{m}$ basis (where $\eta^{\prime \alpha}=d x^{\alpha}+a^{\prime \alpha}{ }_{m}$ ), this becomes

$$
\begin{aligned}
B_{(2)}^{\prime \mathrm{bg}} & =\frac{1}{2} H_{(3) \underline{\alpha n r}}^{\prime} x^{r} \eta^{\prime \alpha} \wedge d x^{n}+\left(\frac{1}{3 !} H_{(3) \underline{m n r}}^{\prime}-\frac{1}{2} H_{(3) \underline{\alpha n r}}^{\prime} a_{m}^{\prime \alpha}\right) x^{r} d x^{m} \wedge d x^{n}, \\
C_{(2)}^{\prime \mathrm{bg}} & =\frac{1}{2} F_{(3) \underline{\alpha \delta r}}^{\prime} x^{r} \eta^{\prime \alpha} \wedge \eta^{\prime \delta}+\left(\frac{1}{2} F_{(3) \underline{\alpha n r}}^{\prime}-F_{(3) \underline{\alpha \delta r}}^{\prime} a_{n}^{\prime \delta}\right) x^{r} \eta^{\prime \alpha} \wedge d x^{n} \\
& +\left(\frac{1}{3 !} F_{(3) \underline{m n r}}^{\prime}-\frac{1}{2} F_{(3) \underline{\alpha n r}}^{\prime} a_{m}^{\prime \alpha}+\frac{1}{2} F_{(3) \underline{\alpha \delta r}}^{\prime} \alpha_{m}^{\prime \alpha} a_{n}^{\prime \delta}\right) x^{r} d x^{m} \wedge d x^{n} .
\end{aligned}
$$

In addition, there are background components of $C_{(4)}^{\text {bg }}$ with one and two $\eta^{\prime \alpha}$ indices, such that

$$
\widetilde{F}_{(5) \text { smeared }}^{\prime}=d C_{(4)}^{\text {bg }}-C_{(2)}^{\text {bg }} \wedge H_{(3)}^{\prime},
$$

with $\widetilde{F}_{(5)}^{\prime}$ given by Eq. (5.30).

The moduli are $c_{(0)}^{\prime}$ and $c_{(4)}^{\prime}=C_{(4)}^{\prime}-C_{(4)}^{\prime \text { bg }}$. The latter are given by

$$
\begin{aligned}
c_{(4)}^{\prime} & =\frac{1}{2 ! 2 !} c_{(4) \underline{\alpha \delta r s}}^{\prime} d x^{\alpha} \wedge d x^{\delta} \wedge d x^{r} \wedge d x^{s}+\frac{1}{3 !} c_{(4) \underline{\alpha n r s}}^{\prime} d x^{\alpha} \wedge d x^{n} \wedge d x^{r} \wedge d x^{s} \\
& +\frac{1}{4 !} c_{(4) m n r s}^{\prime} d x^{m} \wedge d x^{n} \wedge d x^{r} \wedge d x^{s} .
\end{aligned}
$$

In the $\eta^{\alpha}, d x^{m}$ basis, this becomes

$$
\begin{aligned}
c_{(4)}^{\prime} & =\frac{1}{2 ! 2 !} c_{(4) \underline{\alpha \delta r s}}^{\prime} \eta^{\prime \alpha} \wedge \eta^{\prime \delta} \wedge d x^{r} \wedge d x^{s} \\
& +\frac{1}{3 !}\left(c_{(4) \underline{\alpha n r s}}^{\prime}-\frac{1}{2} c_{(4) \underline{\alpha \delta r s}}^{\prime} a^{\prime \delta}{ }_{n}\right) \eta^{\prime \alpha} \wedge d x^{n} \wedge d x^{r} \wedge d x^{s} \\
& +\left(\frac{1}{4 !} c_{(4) m n r s}^{\prime}-\frac{1}{3 !} c_{(4) \underline{\alpha n r s}}^{\prime} a^{\prime \alpha}{ }_{n}+\frac{1}{2 ! 2 !} c_{(4) \underline{\alpha \delta r s}}^{\prime} a^{\prime \alpha}{ }_{m} a^{\prime \beta}{ }_{n}\right) d x^{m} \wedge d x^{n} \wedge d x^{r} \wedge d x^{s} .
\end{aligned}
$$

Applying the T-duality map ([F.1), replacing $a_{(2)}^{\prime}$ with $-b_{(2)}$ via Eq. (5.25a), and at the same time using the flux relations (5.31), we obtain

$$
\begin{aligned}
C_{(0)} & =F_{(1) m} x^{m} \\
C_{(2) 45} & =\widetilde{c}_{(2) 45} \\
C_{(2) \alpha n} & =\frac{1}{2} F_{(3) \alpha n r}^{\mathrm{bg}} x^{r}+b_{\alpha n} C_{(0)}, \\
C_{(2) m n} & =C_{(2) m n}^{\mathrm{bg}}+\widetilde{c}_{(2) m n} \\
C_{(4) \alpha n r s} & =C_{(4) \alpha n r s}^{\mathrm{bg}}+\widetilde{c}_{(4) \alpha n r s}-\left(b_{(2)} \wedge \widetilde{c}_{(2)}\right)_{\alpha n r s}, \\
C_{(4) 45 m n} & =F_{(5) 45 m n r}^{\mathrm{bg}} x^{r}+\left(b_{(2)} \wedge \frac{1}{2} F_{(3) 5 q p} x^{p} d x^{q}\right)_{45 m n}+\frac{1}{2}\left(b_{(2)} \wedge b_{(2)} C_{(0)}\right)_{45 m n}, \\
C_{(6) 45 m n r s} & =\widetilde{c}_{(6) 45 m n r s}+\left(b_{(2)} \wedge \widetilde{c}_{(4)}\right)_{45 m n r s}+\frac{1}{2}\left(b_{(2)} \wedge b_{(2)} \wedge \widetilde{c}_{(2)}\right)_{45 m n r s},
\end{aligned}
$$


where

$$
C_{(2) r s}^{\mathrm{bg}}=C_{(4) 45 r s}^{\mathrm{bg}}, \quad C_{(4) \alpha n r s}^{\mathrm{bg}}=-\epsilon^{\alpha \beta} C_{(4) \alpha n r s}^{\mathrm{bg}},
$$

and where the T-duality map of RR moduli is

$$
\begin{aligned}
& \widetilde{c}_{(2) \underline{45}}=-c_{(0)}^{\prime}, \quad \widetilde{c}_{(2) r s}=c_{(4) \underline{45 r s}}^{\prime}, \\
& \widetilde{c}_{(4) \alpha n r s}=-\epsilon^{\alpha \beta} c_{(4) \underline{\beta n r s}}^{\prime}, \quad \widetilde{c}_{(6) 456789}=c_{(4) \underline{6789}}^{\prime} .
\end{aligned}
$$

Eq. (F.7) is of the same form as Eqs. (3.43) and (3.44), with the gauge choice

$$
C_{(0)}=F_{(1) m} x^{m}, \quad C_{(2) \alpha n}^{\mathrm{bg}}=\frac{1}{2} F_{(3) \alpha n r}^{\mathrm{bg}} x^{r}, \quad C_{(4) 45 m n}^{\mathrm{bg}}=F_{(5) 45 m n r}^{\mathrm{bg}} x^{r} .
$$

Similarly, from Eqs. (5.25b, $)$, the gauge choices for $\mathcal{A}^{\alpha}$ and $B_{(2)}^{\mathrm{bg}}$ resulting from Eq. (F.2) and the T-duality map are

$$
\mathcal{A}^{\alpha}=\frac{1}{2} \mathcal{F}_{m n}^{\alpha} x^{m} d x^{n}, \quad B_{(2)}^{\mathrm{bg}}=\frac{1}{3 !} H_{(3) m n r} x^{m} d x^{n} \wedge d x^{r}
$$

One can check that the potentials $(\mathbb{F . 7})$ do indeed give rise to the fluxes (3.200), (3.47), and $(3.50)$.

\section{Appendix G. Moduli Space Metrics}

In this appendix, we discuss the kinetic terms for the moduli of the $T^{6} / \mathbb{Z}_{2}$ O3 orientifold. We restrict to the $\mathcal{N}=2$ flux (6.55) of Sec. 6.4 and the $\mathcal{N}=1$ flux (8.24) of Sec. 8.2, and work to leading order in $Z-1.43$ For the $\mathcal{N}=3$ case, the kinetic terms were derived by Frey and Polchinski [60], and shown to describe a sigma model with target (7.37), as required by Ref. [77]. Here, we follow Frey and Polchinski, except for a minor difference in conventions, 4 and the inclusion of the axion-dilaton modulus.

43 Properly treating the warp factor in the $4 \mathrm{D}$ kinetic terms for the $6 \mathrm{D}$ metric moduli is a problem that we do not attempt to address here. It was partially studied in Ref. [13], however, the treatment there neglected terms involving $\partial_{\mu} Z=\left(\partial Z / \partial g_{a b}\right) \partial_{\mu} g_{a b}$. It is is currently under investigation 61.

44 We take $x^{a} \cong x^{a}+1$ on the $T^{6}$, whereas Frey and Polchinski take $x^{a} \cong x^{a}+2 \pi$. So, some coefficients differ by powers of $2 \pi$. In addition, we do not absorb factors $g_{s}=e^{\langle\phi\rangle}$ into the normalizations of the $4 \mathrm{D}$ fields, since, in the $\mathcal{N}=2$ case, $\phi$ is an unlifted modulus whose vev is not a priori determined. 
The $10 \mathrm{D}$ string frame action for the metric, dilaton, and axion $C_{(0)}$ is

$$
S_{G, \phi, C_{(0)}}^{10 S}=\frac{1}{2 \cdot 2 \kappa_{10}^{2}} \int d^{10} x \sqrt{-G_{S}}\left(e^{-2 \phi}\left(R_{10 \mathrm{~S}}+4(\partial \phi)^{2}\right)-\frac{1}{2}\left(\partial C_{(0)}\right)^{2}\right),
$$

where $2 \kappa_{10}^{2}=(2 \pi)^{6} \alpha^{\prime 4}$ and $\left(G_{\mathrm{S}}\right)_{M N}$ denotes the metric (5.1a). The integral runs over the $\mathbb{Z}_{2}$ covering space $\mathbb{R}^{3,1} \times T^{6}$, so there is an overall factor of $1 / 2$ multiplying the action. In the $10 \mathrm{D}$ Einstein frame,

$$
\left(G_{\mathrm{E}}\right)_{M N}=e^{-\phi / 2}\left(G_{\mathrm{S}}\right)_{M N}
$$

the action becomes

$$
S_{G, \tau_{\mathrm{dil}}}^{10 \mathrm{E}}=\frac{1}{4 \kappa_{10}^{2}} \int d^{10} x \sqrt{-G_{E}}\left(R_{10 \mathrm{E}}-\frac{\partial_{M} \tau_{\mathrm{dil}} \partial^{M} \bar{\tau}_{\mathrm{dil}}}{2\left(\operatorname{Im} \tau_{\mathrm{dil}}\right)^{2}}\right)
$$

where now the contractions are performed using the new metric $G_{\mathrm{E}}$. The dimensional reduction down to $4 \mathrm{D}$ is

$$
S_{G, \tau_{\mathrm{dil}}}^{4}=\frac{1}{2 \pi \alpha^{\prime}} \int d^{4} x \sqrt{-G_{4}} \Delta\left(R_{4}-\frac{\partial_{\mu} \tau_{\mathrm{dil}} \partial^{\mu} \bar{\tau}_{\mathrm{dil}}}{2\left(\operatorname{Im} \tau_{\mathrm{dil}}\right)^{2}}-\frac{\partial_{\mu} \Delta \partial^{\mu} \Delta}{2 \Delta^{2}}-\frac{1}{4} G^{a c} G^{b d} \partial_{\mu} G_{a b} \partial^{\mu} G_{c d}\right) .
$$

Here, $(2 \pi)^{6} \alpha^{\prime 3} \Delta=\left(\operatorname{det} G_{6}\right)^{1 / 2} / 2$, with $G_{4}$ and $G_{6}$ the restrictions of $G_{\mathrm{E}}$ to $4 \mathrm{D}$ and $6 \mathrm{D}$, respectively. Finally, in terms of the $4 \mathrm{D}$ Einstein frame metric $\left(g_{\mathrm{E}}\right)_{\mu \nu}=\left(G_{4}\right)_{\mu \nu} \Delta$ and rescaled $6 \mathrm{D}$ metric $\gamma_{a b}=\left(G_{6}\right)_{a b} /\left((2 \pi)^{2} \alpha^{\prime} \Delta\right)$, this becomes

$$
S_{g, \tau_{\mathrm{dil}}, \gamma}^{4 \mathrm{E}}=\frac{1}{2 \pi \alpha^{\prime}} \int d^{4} x \sqrt{-g_{\mathrm{E}}}\left(R_{\mathrm{E}}-\frac{\partial_{\mu} \tau_{\mathrm{dil}} \partial^{\mu} \bar{\tau}_{\mathrm{dil}}}{2\left(\operatorname{Im} \tau_{\mathrm{dil}}\right)^{2}}-\frac{1}{4} \gamma^{a c} \gamma^{b d} \partial_{\mu} \gamma_{a b} \partial^{\mu} \gamma_{c d}\right)
$$

As in Ref. [60, once the moduli $c_{(4) a b c d}$ and $\Phi_{I}{ }^{a}$ are included, the total 4D Einstein-Hilbert plus moduli action is

$$
\begin{gathered}
S_{g, \tau_{\mathrm{dil}}, \alpha, \beta, \gamma}^{4 \mathrm{E}}=S_{g}^{4 \mathrm{E}}+S_{\tau_{\mathrm{dil}}}^{4 \mathrm{E}}+S_{\alpha, \beta, \gamma}^{4 \mathrm{E}}, \quad \text { where } \\
S_{g}^{4 \mathrm{E}}=\frac{1}{2 \pi \alpha^{\prime}} \int d^{4} x \sqrt{-g_{\mathrm{E}}} R_{\mathrm{E}}, \\
S_{\tau_{\mathrm{dil}}}^{4 \mathrm{E}}=-\frac{1}{2 \pi \alpha^{\prime}} \int d^{4} x \sqrt{-g_{\mathrm{E}}} \frac{\partial_{\mu} \tau_{\mathrm{dil}} \partial^{\mu} \bar{\tau}_{\mathrm{dil}}}{2\left(\operatorname{Im} \tau_{\mathrm{dil}}\right)^{2}}, \\
S_{\alpha, \beta, \gamma}^{4 \mathrm{E}}=-\frac{1}{2 \pi \alpha^{\prime}} \int d^{4} x \sqrt{-g_{\mathrm{E}}}\left(\gamma_{a b} \partial_{\mu} \alpha_{I}{ }^{a} \partial^{\mu}{\alpha_{I}}^{b}+\frac{1}{4} \gamma_{a c} \gamma_{b d}\left(\mathcal{D}_{\mu} \beta^{a b} \mathcal{D}^{\mu} \beta^{c d}+\partial_{\mu} \gamma^{a b} \partial^{\mu} \gamma^{c d}\right)\right) .
\end{gathered}
$$


In Eq. (G.60),

$$
\begin{aligned}
\alpha_{I}^{a} & =\Phi_{I}^{a} /(2 \pi), \\
c_{(4) a b c d} & =(2 \pi)^{4} \alpha^{\prime 2} \epsilon_{a b c d e f} \beta^{e f}, \quad \text { where } \quad \epsilon_{456789}=1,
\end{aligned}
$$

and

$$
\mathcal{D}_{\mu} \beta^{a b}=\partial_{\mu} \beta^{a b}+\alpha_{I}{ }^{[a \mid} \partial_{\mu} \alpha_{I}{ }^{\mid b]}
$$

The second term in $\mathcal{D}_{\mu} \beta^{a b}$ is the analog of the gauge Chern-Simons term in $F_{(3)}$ of type I or in $H_{(3)}$ of the heterotic theories. It is well known that the sigma model (G.6d) with $a, b$ running over $D$ values and $I$ running over $M$ values parametrizes the coset $\mathcal{T}_{D, D+M}$, where $\mathcal{T}_{m, n}$ is the Grassmannian

$$
\mathcal{T}_{m, n}=\frac{S O(m, n)}{S O(m) \times S O(n)}
$$

up to discrete identifications [85].

Thus, for $\mathcal{N}=4$, with $2 M=32$ D3 branes and no flux, the scalar manifold of the low energy effective field theory is

$$
\mathcal{M}_{\mathcal{N}=4}=\frac{S U(1,1)}{U(1)} \times \frac{S O(6,22)}{S O(6) \times S O(22)}
$$

up to discrete identifications, where the first factor is from $\tau_{\text {dil }}$ and the second from $\alpha, \beta, \gamma$. When there is nonvanishing flux, the supersymmetry is reduced, and the moduli constraints reduce this moduli space to a proper submanifold that is also a product of homogenous spaces.

For $\mathcal{N}=3$ flux, the axion-dilaton and some $\beta, \gamma$ moduli are fixed. In the $w$-coordinates of Sec. 8.2, the unlifted components of $\alpha, \beta, \gamma$ are $\alpha_{I}{ }^{i}, \alpha_{I}{ }^{\bar{\imath}}, \beta^{i \bar{\jmath}}=-\beta^{\bar{\jmath} i}, \gamma^{i \bar{\jmath}}=\gamma^{\bar{\jmath} i}$, and the above sigma model parametrizes the coset $U(3,3+M) /(U(3) \times U(3+M))$, as discussed in Refs. [77,60].

For the $\mathcal{N}=1$ flux of Sec. 8.2, the moduli constraints are similar. The axion-dilaton is again fixed. In the $z$-coordinates of Sec. 8.2, the nonvanishing components of $\alpha, \beta$, $\gamma$ are as in the $\mathcal{N}=3$ case in $w$-coordinates, but with the addition restriction that $\beta^{i \bar{\jmath}}$ and $\gamma^{i \bar{\jmath}}$ be symmetric (imaginary and real, respectively). That is, $\beta^{i \bar{\jmath}}=\beta^{j \bar{\imath}}$ and $\gamma^{i \bar{\jmath}}=$ $\gamma^{j \bar{\imath}}$. It would be interesting to describe this $6+6 M$ dimensional real hypersurface in $U(3,3+M) /(U(3) \times U(3+M))$ as a coset, perhaps $U(3,1+M) /(U(3) \times U(1+M))$. 
Finally, for the $\mathcal{N}=2$ flux of Sec. 6.4, the moduli constraints are such that the scalar manifold factorizes into an $a=4,5,6,7$ part and an $a=8,9$ part. If there were no further constraints, the moduli space would be $(S U(1,1) / U(1)) \times \mathcal{T}_{2,2+M} \times \mathcal{T}_{4,4+M}$. The first two factors form the manifold $\mathcal{S T}_{2,2+M}$ in the special Kähler series

$$
\mathcal{S T}_{2, n}=\frac{S U(1,1)}{U(1)} \times \frac{S O(2, n)}{S O(2) \times S O(n)} .
$$

The third factor is quaternionic. Therefore, we would identify the vector multiplet moduli space with $\mathcal{S T}_{2,2+M}$ and the hypermultiplet moduli space with $\mathcal{T}_{4,4+M}$. However, there are additional moduli constraints that further reduce these special Kähler and quaternionic manifolds. First, there is the constraint $(m / n)\left(-1 / \tau_{\text {dil }}\right)=\tau_{3}^{\prime}$, where $\tau_{3}^{\prime}$ is the complex structure modulus of $\gamma_{a b}$ in the 8,9 directions. Therefore, $\mathcal{S} \mathcal{T}_{2,2+M}$ is further lifted to 45

$$
\mathcal{M}_{V}=\mathcal{S T}_{2,1+M}
$$

If, in the 4, 5, 6, 7 directions, we write (cf. Eqs. (6.63a,b) )

$$
\gamma_{a b} d x^{a} d x^{b}=\frac{\gamma_{1}}{\operatorname{Im} \tau_{1}^{\prime}}\left|\eta^{4}+\tau_{1}^{\prime} \eta^{\prime 5}\right|^{2}+\frac{\gamma_{2}}{\operatorname{Im} \tau_{2}^{\prime}}\left|d x^{6}+\tau_{2}^{\prime} d x^{7}\right|^{2}
$$

with $\eta^{\prime 4}, \eta^{\prime 5}$ given by Eqs. $\left.6.64 a, b\right)$, then the remaining moduli constraints are

$$
\tau_{1}^{\prime} \tau_{2}^{\prime}=-1, \quad a^{\prime 4}=a_{6}{ }_{6}, \quad \text { and } \quad \beta^{46}=-\beta^{57} .
$$

The hypermultiplet moduli space $\mathcal{M}_{H}$ is the hypersurface (G.13) in $\mathcal{T}_{4,4+M}$. Since it must be quaternionic, we conclude that 46

$$
\mathcal{M}_{H}=\mathcal{T}_{4,3+M}
$$

As we have already observed in Secs. 6.4 and 8.2, for classes of flux discussed in this paper, the partial breaking of $\mathcal{N}=4$ to $\mathcal{N}=1,2$ supersymmetry is such that the resulting

45 The only other homogeneous symmetric space $\mathcal{G} / \mathcal{H}$ of dimension $2(2+M)$, with $M$ divisible by four, that is special Kähler is $U(1,2+M) /(U(1) \times U(2+M))$. However, this is not a hypersurface in $\mathcal{S} \mathcal{T}_{2,2+M}$. For a nice review of special Kähler and hyperKähler/quaternionic geometry in connection with both locally and globally $\mathcal{N}=2$ supersymmetric field theories, see Ref. [86].

46 Likewise, the only other homogeneous symmetric quaternionic manifolds of dimension $4(3+$ $M)$ are $S p(2 M+8) /(S p(2) \times S p(2 M+6))$ and $U(2,3+M) /(U(2) \times U(3+M))$. Neither of these is a hypersurface in $\mathcal{T}_{4,4+M}$. 
massive gravitini all have equal mass. It is noteworthy that for the $T^{6} / \mathbb{Z}_{2} \mathrm{O} 3$ orientifold, there does not exist an single proper example in the literature (that is, a choice of flux consistent with Dirac quantization and the Gauss's law constraint) in which the breaking involves massive gravitini of unequal mass. The "generic" case of partial supersymmetry breaking with unequal gravitino mass is apparantly not as generic as one might have thought. It is not clear that such a case is possible. However, if it is, then the resulting mass spectra and superHiggs mechanism would be governed by the results of Ref. [17], in which the reduced moduli spaces for this case have also been worked out.

\section{Appendix H. Uniqueness of Flux in Sec. 8.2}

In this appendix we prove the claim that the flux (8.23) is the unique choice of supersymmetric flux in the class $(8.17 a, b)$ with the minimal value $N_{\text {flux }}=12$, modulo $S L(2, \mathbb{Z})_{\tau} \times S L(2, \mathbb{Z})_{\tau_{\text {dil }}}$ equivalences. (Here $S L(2, \mathbb{Z})_{\tau}$ is the diagonal subgroup of $S L(2, \mathbb{Z})^{3} \subset S L(6, \mathbb{Z})$, where $S L(6, \mathbb{Z})$ is the duality group of $T^{6}$, and $S L(2, \mathbb{Z})^{3}$ is the product of the duality groups of the $T^{2}$ factors in the $T^{6} \rightarrow T^{2} \times T^{2} \times T^{2}$ factorization of the complex structure for this example.) For notational simplicity, we drop the primes on $\tau$ and $\tau_{\text {dil }}$ that were used in Sec. 8.2 solely to denote that O3 from O5 quantities. Instead, primes will indicate $S L(2, \mathbb{Z})$ transformed quantities below. It is convenient to rewrite Eq. (7.27) in matrix form as

$$
P(\tau)=\frac{1}{2}\left(\begin{array}{ll}
\tau & 1
\end{array}\right) \hat{P}\left(\begin{array}{l}
\tau \\
1
\end{array}\right), \quad \text { where } \quad \hat{P}=\left(\begin{array}{cc}
2 l & m \\
m & 2 n
\end{array}\right) .
$$

Then,

$$
\operatorname{det} \hat{P}=4 l n-m^{2},
$$

and under $S L(2, \mathbb{Z})_{\tau}$ transformations,

$$
\begin{aligned}
& \tau \rightarrow \tau^{\prime}, \quad \text { where } \quad\left(\begin{array}{c}
\tau \\
1
\end{array}\right)=\frac{1}{\gamma \tau^{\prime}+\delta} M\left(\begin{array}{c}
\tau^{\prime} \\
1
\end{array}\right), \\
& \hat{P} \rightarrow \hat{P}^{\prime}, \quad \text { where } \quad \hat{P}^{\prime}=M^{T} \hat{P} M,
\end{aligned}
$$

with

$$
M=\left(\begin{array}{ll}
\alpha & \beta \\
\gamma & \delta
\end{array}\right) \in S L(2 \mathbb{Z})_{\tau}
$$


For $N_{\text {flux }}=12$, we have $(f k-g h)\left(4 l n-m^{2}\right)=9$. Since $4 l n-m^{2} \equiv-1(\bmod 4)$, the only possible factorization is

$$
f k-g h=3, \quad 4 l n-m^{2}=3 .
$$

Here we have used the fact that $4 l n-m^{2}>0$ in order for $P(\tau)$ to have two complex conjugate roots with nonzero imaginary part. This is required for the $T^{6}$ to be nondegenerate.

It is well known that $\tau$ can also be mapped into the fundamental domain (7.30) by $S L(2, \mathbb{Z})_{\tau}$ transformations. For $\tau \in \mathcal{F}_{0}$, the conditions $(7.32)$ combined with the second equality in Eq. (H.4) imply that $3 \geq 4 l(n-l)$, with $n \geq l>0$. Therefore, $l=n$. From Eq. (․․ $)$, we then have $3=(2 l+|m|)(2 l-|m|)$. So, $l= \pm m=n=1$, corresponding to $\tau=\frac{1}{2}(\mp 1+i \sqrt{3})$ from Eq. (7.32). The two possibilities are dual via $\tau \rightarrow \tau \pm 1$. (Only the $m=+1$ solution is actually in the fundamental domain as defined in (7.30)). Thus,

$$
(l, m, n)=(1,1,1) \quad \text { and } \quad \tau=e^{2 \pi i / 3}, \quad \text { up to } S L(2, \mathbb{Z})_{\tau} \text { duality. }
$$

Now consider the equivalences on $(f, g, h, k)$. Under $S L(2, \mathbb{Z})_{\tau_{\text {dil }}}$ duality,

$$
A=\left(\begin{array}{ll}
f & g \\
h & k
\end{array}\right) \rightarrow A^{\prime}=N A, \quad \text { where } \quad N \in S L(2, \mathbb{Z})_{\tau_{\mathrm{dil}}} .
$$

From $a=b=-\frac{2}{3}(f+g), c=d=-\frac{2}{3}(h+k)$, together with $f k-g h=3$, we conclude that $\operatorname{gcd}(f, h)=1$. (Assume, to the contrary, that $\operatorname{gcd} f, h=3$. Then, demanding that $a, b, c, d \in 2 \mathbb{Z}$ implies that $\operatorname{gcd}(g, k)=3$ and subsequently that 9 divides $f k-g h$. This contradicts $f k-g h=3$.) Therefore, $\gamma f+\delta h=1$ for some $\gamma, \delta$, so that the $S L(2, \mathbb{Z})_{\tau_{\mathrm{dil}}}$ duality $A^{\prime}=\left(\begin{array}{cc}f^{\prime} & \gamma^{\prime} \\ h^{\prime} & k^{\prime}\end{array}\right)=\left(\begin{array}{cc}h & -f \\ \gamma & \delta\end{array}\right)\left(\begin{array}{ll}f & g \\ h & k\end{array}\right)$ gives $h^{\prime}=1$. Next, the duality $A^{\prime \prime}=\left(\begin{array}{cc}f^{\prime \prime} & g^{\prime \prime} \\ h^{\prime \prime} & k^{\prime \prime}\end{array}\right)=\left(\begin{array}{ccc}0 & 1 \\ -1 & f^{\prime}\end{array}\right)\left(\begin{array}{cc}f^{\prime} & g^{\prime} \\ 1 & k^{\prime}\end{array}\right)$ gives $h^{\prime \prime}=0$. Finally, the $S L(2, \mathbb{Z})_{\tau_{\text {dil }}}$ duality $A^{\prime \prime \prime}=\left(\begin{array}{ll}f^{\prime \prime \prime} & g^{\prime \prime \prime} \\ 0 & k^{\prime \prime \prime}\end{array}\right)=\left(\begin{array}{ll}1 & \beta \\ 0 & 1\end{array}\right)\left(\begin{array}{ll}f^{\prime \prime} & g^{\prime \prime} \\ 0 & k^{\prime \prime}\end{array}\right)$ allows us to replace $g^{\prime \prime}$ with any $g^{\prime \prime \prime} \equiv g^{\prime \prime}\left(\bmod k^{\prime \prime}\right)$. From $\operatorname{det} A^{\prime \prime \prime}=\operatorname{det} A^{\prime \prime}=\operatorname{det} A^{\prime}=\operatorname{det} A=3$, we have $f^{\prime \prime \prime} k^{\prime \prime \prime}=3$. We can assume that $f^{\prime \prime \prime}, k^{\prime \prime \prime}>0$ (via $S L(2, \mathbb{Z})$ duality by $\left(\begin{array}{cc}-1 & 0 \\ 0 & -1\end{array}\right)$, if necessary). There are two possible factorizations: $f^{\prime \prime \prime}=1$ and $k^{\prime \prime \prime}=3$, or $f^{\prime \prime \prime}=3$ and $k^{\prime \prime \prime}=1$. The second case is excluded by $c=d=-2 k^{\prime \prime \prime} / 3 \in \mathbb{Z}$. In the first case, the congruence $-3 a=2\left(f^{\prime \prime \prime}+g^{\prime \prime \prime}\right) \equiv 0(\bmod 3)$ gives $g^{\prime \prime \prime} \equiv-1(\bmod 3) \equiv-1\left(\bmod k^{\prime \prime \prime}\right)$. So, $g^{\prime \prime \prime}=-1$ and $\left(\begin{array}{cc}f & g \\ h & k\end{array}\right)=\left(\begin{array}{cc}1 & -1 \\ 0 & 3\end{array}\right)$ up to $S L(2, \mathbb{Z})_{\tau_{\text {dil }}}$ duality. This is equivalent to

$$
\left(\begin{array}{ll}
f & g \\
h & k
\end{array}\right)=\left(\begin{array}{cc}
1 & -1 \\
1 & 2
\end{array}\right) \quad \text { up to } S L(2, \mathbb{Z})_{\tau_{\mathrm{dil}}} \text { duality. }
$$

Together, Eqs. (H.5) and (H.7) prove the desired uniqueness of the choice (8.23b). 


\section{References}

[1] C. M. Hull, "Superstring Compactifications With Torsion And Space-Time Supersymmetry," Print-86-0251 (CAMBRIDGE).

[2] A. Strominger, "Superstrings With Torsion," Nucl. Phys. B 274, 253 (1986).

[3] K. Dasgupta, G. Rajesh and S. Sethi, "M theory, orientifolds and G-flux," JHEP 9908, 023 (1999) arXiv:hep-th/9908088.

[4] S. Kachru, M. B. Schulz, P. K. Tripathy and S. P. Trivedi, "New supersymmetric string compactifications," JHEP 0303, 061 (2003) arXiv:hep-th/0211182.

[5] P. Candelas, G. T. Horowitz, A. Strominger and E. Witten, "Vacuum Configurations For Superstrings," Nucl. Phys. B 258, 46 (1985).

[6] M. Graña and J. Polchinski, "Gauge/gravity duals with holomorphic dilaton," Phys. Rev. D 65, 126005 (2002) arXiv:hep-th/0106014.

[7] H. Verlinde, "Holography and compactification," Nucl. Phys. B 580, 264 (2000) arXiv:hep-th/9906182;

C. S. Chan, P. L. Paul and H. Verlinde, "A note on warped string compactification," Nucl. Phys. B 581, 156 (2000) arXiv:hep-th/0003236.

[8] I. R. Klebanov and M. J. Strassler, "Supergravity and a confining gauge theory: Duality cascades and $\chi$ SB-resolution of naked singularities," JHEP 0008, 052 (2000) arXiv:hep-th/0007191.

[9] S. B. Giddings, S. Kachru and J. Polchinski, "Hierarchies from fluxes in string compactifications," Phys. Rev. D 66, 106006 (2002) arXiv:hep-th/0105097.

[10] L. Randall and R. Sundrum, 'A large mass hierarchy from a small extra dimension," Phys. Rev. Lett. 83, 3370 (1999) arXiv:hep-ph/9905221;

L. Randall and R. Sundrum, "An alternative to compactification," Phys. Rev. Lett. 83, 4690 (1999) arXiv:hep-th/9906064.

[11] S. Kachru, M. B. Schulz and S. Trivedi, "Moduli stabilization from fluxes in a simple IIB orientifold," JHEP 0310, 007 (2003) arXiv:hep-th/0201028].

[12] P. K. Tripathy and S. P. Trivedi, "Compactification with flux on $K 3$ and tori," JHEP 0303, 028 (2003) arXiv:hep-th/0301139;

A. Giryavets, S. Kachru, P. K. Tripathy and S. P. Trivedi, "Flux compactifications on Calabi-Yau threefolds," JHEP 0404, 003 (2004) arXiv:hep-th/0312104.

[13] O. DeWolfe and S. B. Giddings, "Scales and hierarchies in warped compactifications and brane worlds," Phys. Rev. D 67, 066008 (2003) arXiv:hep-th/0208123.

[14] J. Polchinski and A. Strominger, "New Vacua for Type II String Theory," Phys. Lett. B 388, 736 (1996) arXiv:hep-th/9510227;

T. R. Taylor and C. Vafa, "RR flux on Calabi-Yau and partial supersymmetry breaking," Phys. Lett. B 474, 130 (2000) arXiv:hep-th/9912152;

P. Mayr, "On supersymmetry breaking in string theory and its realization in brane 
worlds," Nucl. Phys. B 593, 99 (2001) arXiv:hep-th/0003198;

B. R. Greene, K. Schalm and G. Shiu, "Warped compactifications in M and F theory," Nucl. Phys. B 584, 480 (2000) arXiv:hep-th/0004103;

G. Curio, A. Klemm, D. Lüst and S. Theisen, "On the vacuum structure of type II string compactifications on Calabi-Yau spaces with $H$-fluxes," Nucl. Phys. B 609, 3 (2001) arXiv:hep-th/0012213;

G. Curio, A. Klemm, B. Körs and D. Lüst, "Fluxes in heterotic and type II string compactifications," Nucl. Phys. B 620, 237 (2002) [arXiv:hep-th/0106155];

G. Curio, B. Körs and D. Lüst, "Fluxes and branes in type II vacua and M-theory geometry with $G_{2}$ and Spin(7) holonomy," Nucl. Phys. B 636, 197 (2002) arXiv:hepth/0111165];

J. Louis and A. Micu, "Type II theories compactified on Calabi-Yau threefolds in the presence of background fluxes," Nucl. Phys. B 635, 395 (2002) arXiv:hep-th/0202168; K. Becker, M. Becker, M. Haack and J. Louis, "Supersymmetry breaking and $\alpha^{\prime}$-corrections to flux induced potentials," JHEP 0206, 060 (2002) arXiv:hepth/0204254];

A. R. Frey and A. Mazumdar, "3-form induced potentials, dilaton stabilization, and running moduli," Phys. Rev. D 67, 046006 (2003) arXiv:hep-th/0210254;

R. Blumenhagen, D. Lüst and T. R. Taylor, "Moduli stabilization in chiral type IIB orientifold models with fluxes," Nucl. Phys. B 663, 319 (2003) arXiv:hep-th/0303016; M. Berg, M. Haack and B. Körs, "An orientifold with fluxes and branes via T-duality," Nucl. Phys. B 669, 3 (2003) arXiv:hep-th/0305183;

S. P. de Alwis, "On potentials from fluxes," Phys. Rev. D 68, 126001 (2003) arXiv:hep-th/0307084;

J. F. G. Cascales and A. M. Uranga, "Chiral 4d string vacua with D-branes and moduli stabilization," arXiv:hep-th/0311250;

J. F. G. Cascales, M. P. Garcia del Moral, F. Quevedo and A. M. Uranga, "Realistic D-brane models on warped throats: Fluxes, hierarchies and moduli stabilization," JHEP 0402, 031 (2004) arXiv:hep-th/0312051;

D. Lüst, S. Reffert and S. Stieberger, "Flux-induced Soft Supersymmetry Breaking in Chiral Type IIB Orientifolds with D3/D7-Branes," arXiv:hep-th/0406092.

[15] A. R. Frey, "Warped strings: Self-dual flux and contemporary compactifications," arXiv:hep-th/0308156.

[16] G. Dall'Agata, "Type IIB supergravity compactified on a Calabi-Yau manifold with H-fluxes," JHEP 0111, 005 (2001) arXiv:hep-th/0107264;

L. Andrianopoli, R. D'Auria, S. Ferrara and M. A. Lledo, "Super Higgs effect in extended supergravity," Nucl. Phys. B 640, 46 (2002) arXiv:hep-th/0202116;

L. Andrianopoli, R. D'Auria, S. Ferrara and M. A. Lledo, "Gauging of flat groups in four dimensional supergravity," JHEP 0207, 010 (2002) arXiv:hep-th/0203206; 
L. Andrianopoli, R. D'Auria, S. Ferrara and M. A. Lledo, "Duality and spontaneously broken supergravity in flat backgrounds," Nucl. Phys. B 640, 63 (2002) arXiv:hepth/0204145;

R. D'Auria, S. Ferrara and S. Vaula, "N $\mathcal{N}=4$ gauged supergravity and a IIB orientifold with fluxes," New J. Phys. 4, 71 (2002) arXiv:hep-th/0206241;

R. D'Auria, S. Ferrara, M. A. Lledo and S. Vaula, "No-scale $\mathcal{N}=4$ supergravity coupled to Yang-Mills: The scalar potential and super Higgs effect," Phys. Lett. B 557, 278 (2003) arXiv:hep-th/0211027;

S. Ferrara, "Duality, gauging and superHiggs effect in string and M-theory," arXiv:hepth/0211116;

L. Andrianopoli, R. D'Auria, S. Ferrara and M. A. Lledo, " $\mathcal{N}=2$ super-Higgs, $\mathcal{N}=$ 1 Poincaré vacua and quaternionic geometry," JHEP 0301, 045 (2003) arXiv:hepth/0212236];

C. Angelantonj, R. D'Auria, S. Ferrara and M. Trigiante, " $K 3 \times T^{2} / \mathbb{Z}_{2}$ orientifolds with fluxes, open string moduli and critical points," Phys. Lett. B 583, 331 (2004) arXiv:hep-th/0312019;

M. Grana, T. W. Grimm, H. Jockers and J. Louis, "Soft supersymmetry breaking in Calabi-Yau orientifolds with D-branes and fluxes," arXiv:hep-th/0312232;

T. W. Grimm and J. Louis, "The effective action of $\mathcal{N}=1$ Calabi-Yau orientifolds," arXiv:hep-th/0403067.

[17] R. D'Auria, S. Ferrara, F. Gargiulo, M. Trigiante and S. Vaula, " $\mathcal{N}=4$ supergravity Lagrangian for type IIB on $T^{6} / \mathbb{Z}_{2}$ in presence of fluxes and D3-branes," JHEP 0306, 045 (2003) arXiv:hep-th/0303049.

[18] S. Ferrara and M. Porrati, " $\mathcal{N}=1$ no-scale supergravity from IIB orientifolds," Phys. Lett. B 545, 411 (2002) arXiv:hep-th/0207135;

L. Andrianopoli, R. D'Auria, S. Ferrara and M. A. Lledo, "Gauged extended supergravity without cosmological constant: No-scale structure and supersymmetry breaking," Mod. Phys. Lett. A 18, 1001 (2003) arXiv:hep-th/0212141.

[19] A. Lawrence and J. McGreevy, "Local string models of soft supersymmetry breaking," arXiv:hep-th/0401034.

[20] L. Susskind, "The anthropic landscape of string theory," arXiv:hep-th/0302219;

M. R. Douglas, "The statistics of string/M theory vacua," JHEP 0305, 046 (2003) arXiv:hep-th/0303194;

S. Ashok and M. R. Douglas, "Counting flux vacua," arXiv:hep-th/0307049;

T. Banks, M. Dine and E. Gorbatov, "Is there a string theory landscape?," arXiv:hepth/0309170;

M. R. Douglas, "Statistics of string vacua," arXiv:hep-ph/0401004;

M. Dine, "Is there a string theory landscape: Some cautionary notes," arXiv:hepth/0402101; 
M. R. Douglas, B. Shiffman and S. Zelditch, "Critical points and supersymmetric vacua," arXiv:math.cv/0402326;

F. Denef and M. R. Douglas, "Distributions of flux vacua," arXiv:hep-th/0404116;

A. Giryavets, S. Kachru and P. K. Tripathy, "On the taxonomy of flux vacua," arXiv:hep-th/0404243;

L. Susskind, "Supersymmetry breaking in the anthropic landscape," arXiv:hepth/0405189;

M. R. Douglas, "Statistical analysis of the supersymmetry breaking scale," arXiv:hepth/0405279.

[21] S. Kachru, R. Kallosh, A. Linde and S. P. Trivedi, "De Sitter vacua in string theory," Phys. Rev. D 68, 046005 (2003) arXiv:hep-th/0301240.

[22] F. Denef, M. R. Douglas and B. Florea, "Building a better racetrack," arXiv:hepth/0404257.

[23] D. Robbins and S. Sethi, "A barren landscape," arXiv:hep-th/0405011.

[24] A. Saltman and E. Silverstein, "The scaling of the no-scale potential and de Sitter model building," arXiv:hep-th/0402135.

[25] K. Dasgupta, C. Herdeiro, S. Hirano and R. Kallosh, "D3/D7 inflationary model and M-theory," Phys. Rev. D 65, 126002 (2002) arXiv:hep-th/0203019;

M. Fabinger and E. Silverstein, "D-Sitter space: Causal structure, thermodynamics, and entropy," arXiv:hep-th/0304220;

A. R. Frey, M. Lippert and B. Williams, "The fall of stringy de Sitter," Phys. Rev. D 68, 046008 (2003) arXiv:hep-th/0305018;

S. Kachru, R. Kallosh, A. Linde, J. Maldacena, L. McAllister and S. P. Trivedi, "Towards inflation in string theory," JCAP 0310, 013 (2003) arXiv:hep-th/0308055;

C. P. Burgess, R. Kallosh and F. Quevedo, "de Sitter string vacua from supersymmetric D-terms," JHEP 0310, 056 (2003) [arXiv:hep-th/0309187;

E. Silverstein and D. Tong, "Scalar speed limits and cosmology: Acceleration from D-cceleration," arXiv:hep-th/0310221;

J. P. Hsu, R. Kallosh and S. Prokushkin, "On brane inflation with volume stabilization," JCAP 0312, 009 (2003) arXiv:hep-th/0311077;

A. Buchel and R. Roiban, "Inflation in warped geometries," arXiv:hep-th/0311154;

F. Koyama, Y. Tachikawa and T. Watari, "Supergravity analysis of hybrid inflation model from D3-D7 system," Phys. Rev. D 69, 106001 (2004) arXiv:hep-th/0311191; P. G. Camara, L. E. Ibanez and A. M. Uranga, "Flux-induced SUSY-breaking soft terms," arXiv:hep-th/0311241;

H. Firouzjahi and S. H. H. Tye, "Closer towards inflation in string theory," Phys. Lett. B 584, 147 (2004) arXiv:hep-th/0312020;

A. Buchel, "On effective action of string theory flux compactifications," Phys. Rev. D 69, 106004 (2004) arXiv:hep-th/0312076; 
R. Brustein and S. P. de Alwis, "Moduli potentials in string compactifications with fluxes: Mapping the discretuum," arXiv:hep-th/0402088;

L. Kofman, A. Linde, X. Liu, A. Maloney, L. McAllister and E. Silverstein, "Beauty is attractive: Moduli trapping at enhanced symmetry points," arXiv:hep-th/0403001; O. DeWolfe, S. Kachru and H. Verlinde, "The giant inflaton," arXiv:hep-th/0403123; N. Iizuka and S. P. Trivedi, "An inflationary model in string theory," arXiv:hepth/0403203;

M. Alishahiha, E. Silverstein and D. Tong, "DBI in the sky," arXiv:hep-th/0404084;

M. Berg, M. Haack and B. Körs, "Loop corrections to volume moduli and inflation in string theory," arXiv:hep-th/0404087;

A. Buchel and A. Ghodsi, "Braneworld inflation," arXiv:hep-th/0404151.

[26] B. de Wit, D. J. Smit and N. D. Hari Dass, "Residual Supersymmetry Of Compactified $D=10$ Supergravity," Nucl. Phys. B 283, 165 (1987);

J. M. Maldacena and C. Nuñez, "Supergravity description of field theories on curved manifolds and a no go theorem," Int. J. Mod. Phys. A 16, 822 (2001) arXiv:hepth/0007018];

S. Ivanov and G. Papadopoulos, "A no-go theorem for string warped compactifications," Phys. Lett. B 497, 309 (2001) [arXiv:hep-th/0008232].

[27] J. P. Gauntlett, D. Martelli and D. Waldram, "Superstrings with intrinsic torsion," arXiv:hep-th/0302158.

[28] J. P. Gauntlett, N. Kim, D. Martelli and D. Waldram, "Fivebranes wrapped on SLAG three-cycles and related geometry," JHEP 0111, 018 (2001) [arXiv:hep-th/0110034];

J. P. Gauntlett, D. Martelli, S. Pakis and D. Waldram, "G-structures and wrapped NS5-branes," arXiv:hep-th/0205050.

[29] G. L. Cardoso, G. Curio, G. Dall'Agata, D. Lüst, P. Manousselis and G. Zoupanos, "Non-Kaehler string backgrounds and their five torsion classes," Nucl. Phys. B 652, 5 (2003) arXiv:hep-th/0211118.

[30] K. Becker and M. Becker, "M-Theory on Eight-Manifolds," Nucl. Phys. B 477, 155 (1996) arXiv:hep-th/9605053.

[31] C. Vafa, "Evidence for F-Theory," Nucl. Phys. B 469, 403 (1996) arXiv:hepth/9602022];

S. Sethi, C. Vafa and E. Witten, "Constraints on low-dimensional string compactifications," Nucl. Phys. B 480, 213 (1996) [arXiv:hep-th/9606122];

K. Dasgupta and S. Mukhi, "A note on low-dimensional string compactifications," Phys. Lett. B 398, 285 (1997) arXiv:hep-th/9612188.

[32] S. Gurrieri, J. Louis, A. Micu and D. Waldram, "Mirror symmetry in generalized Calabi-Yau compactifications," Nucl. Phys. B 654, 61 (2003) arXiv:hep-th/0211102.

[33] J. P. Gauntlett and S. Pakis, "The geometry of $D=11$ Killing spinors," JHEP 0304, 039 (2003) arXiv:hep-th/0212008. 
[34] D. Martelli and J. Sparks, "G-structures, fluxes and calibrations in M-theory," Phys. Rev. D 68, 085014 (2003) arXiv:hep-th/0306225.

[35] J. P. Gauntlett, J. B. Gutowski and S. Pakis, "The geometry of $D=11$ null Killing spinors," JHEP 0312, 049 (2003) [arXiv:hep-th/0311112.

[36] J. P. Gauntlett, D. Martelli, J. Sparks and D. Waldram, "Supersymmetric $A d S_{5}$ solutions of M-theory," arXiv:hep-th/0402153.

[37] P. Kaste, R. Minasian, M. Petrini and A. Tomasiello, "Kaluza-Klein bundles and manifolds of exceptional holonomy," JHEP 0209, 033 (2002) arXiv:hep-th/0206213;

P. Kaste, R. Minasian, M. Petrini and A. Tomasiello, "Nontrivial RR two-form field strength and $S U(3)$-structure," Fortsch. Phys. 51, 764 (2003) arXiv:hep-th/0301063; P. Kaste, R. Minasian and A. Tomasiello, "Supersymmetric M-theory compactifications with fluxes on seven-manifolds and G-structures," JHEP 0307, 004 (2003) arXiv:hep-th/0303127.

[38] S. Fidanza, R. Minasian and A. Tomasiello, "Mirror symmetric $S U(3)$-structure manifolds with NS fluxes," arXiv:hep-th/0311122.

[39] T. Z. Husain, "M2-branes wrapped on holomorphic curves," JHEP 0312, 037 (2003) arXiv:hep-th/0211030;

T. Z. Husain, "That's a wrap!," JHEP 0304, 053 (2003) arXiv:hep-th/0302071.

[40] M. J. Duff and J. T. Liu, "Hidden spacetime symmetries and generalized holonomy in M-theory," Nucl. Phys. B 674, 217 (2003) arXiv:hep-th/0303140;

C. Hull, "Holonomy and symmetry in M-theory," arXiv:hep-th/0305039;

G. Papadopoulos and D. Tsimpis, "The holonomy of the supercovariant connection and Killing spinors," JHEP 0307, 018 (2003) arXiv:hep-th/0306117;

G. Papadopoulos and D. Tsimpis, "The holonomy of IIB supercovariant connection," Class. Quant. Grav. 20, L253 (2003) arXiv:hep-th/0307127;

A. Batrachenko, M. J. Duff, J. T. Liu and W. Y. Wen, "Generalized holonomy of M-theory vacua," arXiv:hep-th/0312165;

A. Batrachenko and W. Y. Wen, "Generalized holonomy of supergravities with 8 real supercharges," arXiv:hep-th/0402141.

[41] G. Dall'Agata and N. Prezas, " $\mathcal{N}=1$ geometries for M-theory and type IIA strings with fluxes," Phys. Rev. D 69, 066004 (2004) arXiv:hep-th/0311146.

[42] G. Dall'Agata, "On supersymmetric solutions of type IIB supergravity with general fluxes," arXiv:hep-th/0403220.

[43] K. Behrndt and C. Jeschek, "Fluxes in M-theory on 7-manifolds and G structures," JHEP 0304, 002 (2003) arXiv:hep-th/0302047;

K. Behrndt and M. Cvetic, "Supersymmetric intersecting D6-branes and fluxes in massive type IIA string theory," Nucl. Phys. B 676, 149 (2004) arXiv:hep-th/0308045;

K. Behrndt and C. Jeschek, "Fluxes in M-theory on 7-manifolds: G-structures and superpotential," arXiv:hep-th/0311119; 
K. Behrndt and M. Cvetic, "General $\mathcal{N}=1$ supersymmetric flux vacua of (massive) type IIA string theory," arXiv:hep-th/0403049.

[44] A. R. Frey, "Notes on $S U(3)$ structures in type IIB supergravity," arXiv:hepth/0404107.

[45] A. Gray and L. M. Hervella, "The sixteen classes of almost Hermitian manifolds and their linear invariants," Ann. Math. Pura Appl. 12335 (1980);

S. Chiossi and S. Salamon, "The Intrinsic Torsion of $S U(3)$ and $G_{2}$ Structures," in Differential Geometry, Valencia 2001, World Sci. Publishing, River Edge, NJ, 115 (2002) arXiv:math.DG/0202282.

[46] J. Gutowski, G. Papadopoulos and P. K. Townsend, "Supersymmetry and generalized calibrations," Phys. Rev. D 60, 106006 (1999) arXiv:hep-th/9905156;

J. Gutowski and G. Papadopoulos, "AdS calibrations," Phys. Lett. B 462, 81 (1999) arXiv:hep-th/9902034;

J. Gutowski, "Generalized calibrations," arXiv:hep-th/9909096.

[47] G. L. Cardoso, G. Curio, G. Dall'Agata and D. Lüst, "BPS action and superpotential for heterotic string compactifications with fluxes," JHEP 0310, 004 (2003) arXiv:hepth/0306088].

[48] K. Becker and K. Dasgupta, "Heterotic strings with torsion," JHEP 0211, 006 (2002) arXiv:hep-th/0209077;

K. Becker, M. Becker, K. Dasgupta and P. S. Green, "Compactifications of heterotic theory on non-Kaehler complex manifolds. I," JHEP 0304, 007 (2003) arXiv:hepth/0301161;

K. Becker, M. Becker, K. Dasgupta and S. Prokushkin, "Properties of heterotic vacua from superpotentials," Nucl. Phys. B 666, 144 (2003) arXiv:hep-th/0304001.

[49] K. Becker, M. Becker, P. S. Green, K. Dasgupta and E. Sharpe, "Compactifications of heterotic strings on non-Kaehler complex manifolds. II," arXiv:hep-th/0310058.

[50] E. Goldstein and S. Prokushkin, "Geometric model for complex non-Kaehler manifolds with $S U(3)$ structure," arXiv:hep-th/0212307.

[51] R. Gopakumar and C. Vafa, "On the gauge theory/geometry correspondence," Adv. Theor. Math. Phys. 3, 1415 (1999) arXiv:hep-th/9811131;

R. Dijkgraaf and C. Vafa, "Matrix models, topological strings, and supersymmetric gauge theories," Nucl. Phys. B 644, 3 (2002) arXiv:hep-th/0206255.

[52] M. Becker, K. Dasgupta, A. Knauf and R. Tatar, "Geometric transitions, flops and non-Kaehler manifolds. I," arXiv:hep-th/0403288.

[53] J. F. G. Cascales and A. M. Uranga, "M5-brane geometries, T-duality and fluxes," JHEP 0401, 021 (2004) arXiv:hep-th/0307156;

M. Serone and M. Trapletti, "String vacua with flux from freely-acting obifolds," JHEP 0401, 012 (2004) arXiv:hep-th/0310245. 
[54] S. Hellerman, J. McGreevy and B. Williams, "Geometric constructions of nongeometric string theories," arXiv:hep-th/0208174;

A. Dabholkar and C. Hull, "Duality twists, orbifolds, and fluxes," JHEP 0309, 054 (2003) arXiv:hep-th/0210209;

A. Flournoy, B. Wecht and B. Williams, "Constructing nongeometric vacua in string theory," arXiv:hep-th/0404217.

[55] J. Scherk and J. H. Schwarz, "How To Get Masses From Extra Dimensions," Nucl. Phys. B 153, 61 (1979).

[56] M. B. Schulz, work in progress.

[57] S. Gukov, C. Vafa and E. Witten, "CFT's from Calabi-Yau four-folds," Nucl. Phys. B 584, 69 (2000) [Erratum-ibid. B 608, 477 (2001)] [arXiv:hep-th/9906070].

[58] C. V. Johnson, "D-brane primer," arXiv:hep-th/0007170.

[59] O. Bergman, E. G. Gimon and S. Sugimoto, "Orientifolds, RR torsion, and K-theory," JHEP 0105, 047 (2001) [arXiv:hep-th/0103183].

[60] A. R. Frey and J. Polchinski, " $\mathcal{N}=3$ warped compactifications," Phys. Rev. D 65, 126009 (2002) arXiv:hep-th/0201029.

[61] S. B. Giddings and A. Maharana, work in progress.

[62] C. Angelantonj, S. Ferrara and M. Trigiante, "New $D=4$ gauged supergravities from $\mathcal{N}=4$ orientifolds with fluxes," JHEP 0310, 015 (2003) arXiv:hep-th/0306185;

C. Angelantonj, S. Ferrara and M. Trigiante, "Unusual gauged supergravities from type IIA and type IIB orientifolds," Phys. Lett. B 582, 263 (2004) arXiv:hepth/0310136;

R. D'Auria, S. Ferrara and M. Trigiante, "Homogeneous special manifolds, orientifolds and solvable coordinates," arXiv:hep-th/0403204.

[63] E. Cremmer, S. Ferrara, C. Kounnas and D. V. Nanopoulos, "Naturally Vanishing Cosmological Constant In $\mathcal{N}=1$ Supergravity," Phys. Lett. B 133, 61 (1983);

J. R. Ellis, A. B. Lahanas, D. V. Nanopoulos and K. Tamvakis, "No-Scale Supersymmetric Standard Model," Phys. Lett. B 134, 429 (1984).

[64] R. C. Myers, "Dielectric-branes," JHEP 9912, 022 (1999) arXiv:hep-th/9910053.

[65] A. R. Frey and M. Grana, "Type IIB solutions with interpolating supersymmetries," Phys. Rev. D 68, 106002 (2003) arXiv:hep-th/0307142.

[66] J. Polchinski and M. J. Strassler, "The string dual of a confining four-dimensional gauge theory," arXiv:hep-th/0003136.

[67] C. N. Gowdigere and N. P. Warner, "Flowing with eight supersymmetries in M-theory and F-theory," JHEP 0312, 048 (2003) arXiv:hep-th/0212190;

C. N. Pope and N. P. Warner, "A dielectric flow solution with maximal supersymmetry," arXiv:hep-th/0304132;

C. N. Gowdigere, D. Nemeschansky and N. P. Warner, "Supersymmetric solutions with fluxes from algebraic Killing spinors," arXiv:hep-th/0306097; 
K. Pilch and N. P. Warner, "Generalizing the $\mathcal{N}=2$ supersymmetric RG flow solution of IIB supergravity," Nucl. Phys. B 675, 99 (2003) arXiv:hep-th/0306098;

K. Pilch and N. P. Warner, " $\mathcal{N}=1$ supersymmetric solutions of IIB supergravity from Killing spinors," arXiv:hep-th/0403005;

D. Nemeschansky and N. P. Warner, "A family of M-theory flows with four supersymmetries," arXiv:hep-th/0403006.

[68] K. Becker, M. Becker and R. Sriharsha, "PP-waves, M-theory and fluxes," Nucl. Phys. B 676, 172 (2004) arXiv:hep-th/0308014.

[69] G. L. Cardoso, G. Curio, G. Dall'Agata and D. Lüst, work in progress.

[70] A. Hanany and B. Kol, "On orientifolds, discrete torsion, branes and M theory," JHEP 0006, 013 (2000) arXiv:hep-th/0003025.

[71] P. Candelas, "Lectures On Complex Manifolds," in Superstrings 87, proceedings of the Trieste Spring School (1987) 1-88;

M. Mariño, R. Minasian, G. W. Moore and A. Strominger, "Nonlinear instantons from supersymmetric p-branes," JHEP 0001, 005 (2000) arXiv:hep-th/9911206.

[72] P. Bouwknegt, J. Evslin and V. Mathai, "T-duality: Topology change from H-flux," arXiv:hep-th/0306062.

[73] P. Bouwknegt, J. Evslin and V. Mathai, "On the topology and $H$-flux of T-dual manifolds," arXiv:hep-th/0312052;

P. Bouwknegt and D. Ridout, "A note on the equality of algebraic and geometric D-brane charges in WZW models," arXiv:hep-th/0312259;

P. Bouwknegt, K. Hannabuss and V. Mathai, "T-duality for principal torus bundles," arXiv:hep-th/0312284;

V. Mathai and J. Rosenberg, "T-duality for torus bundles via noncommutative topology," arXiv:hep-th/0401168.

[74] E. Bergshoeff, C. M. Hull and T. Ortín, "Duality in the type II superstring effective action," Nucl. Phys. B 451, 547 (1995) arXiv:hep-th/9504081.

[75] S. F. Hassan, "T-duality, space-time spinors and R-R fields in curved backgrounds," Nucl. Phys. B 568, 145 (2000) arXiv:hep-th/9907152.

[76] S. Salamon, "Complex structures on nilpotent Lie algebras," arXiv:math.DG/9808025; E. Abbena, S. Garbiero, S. Salamon, "Almost Hermitian Geometry on Six Dimensional Nilmanifolds," arXiv:math.DG/0007066.

[77] L. Castellani, A. Ceresole, S. Ferrara, R. D'Auria, P. Fré and E. Maina, "The Complete $\mathcal{N}=3$ Matter Coupled Supergravity," Nucl. Phys. B 268, 317 (1986).

[78] A. Sen, "A note on enhanced gauge symmetries in M- and string theory," JHEP 9709, 001 (1997) arXiv:hep-th/9707123.

[79] E. Witten, "Non-Perturbative Superpotentials In String Theory," Nucl. Phys. B 474, 343 (1996) arXiv:hep-th/9604030. 
[80] N. Seiberg, "IR dynamics on branes and space-time geometry," Phys. Lett. B 384, 81 (1996) arXiv:hep-th/9606017;

N. Seiberg and E. Witten, "Gauge dynamics and compactification to three dimensions," arXiv:hep-th/9607163.

[81] M. Kreuzer and H. Skarke, "Reflexive polyhedra, weights and toric Calabi-Yau fibrations," Rev. Math. Phys. 14, 343 (2002) arXiv:math.ag/0001106;

M. Kreuzer and H. Skarke, "Complete classification of reflexive polyhedra in four dimensions," Adv. Theor. Math. Phys. 4, 1209 (2002) arXiv:hep-th/0002240.

[82] M. Nakahara, "Geometry, Topology and Physics," (Graduate student series in physics), Institute of Physics Publishing, Bristol, UK (1990).

[83] C. W. Misner, K. S. Thorne and J. A. Wheeler, "Gravitation," W. H. Freeman and Company, New York (2000).

[84] S. J. J. Gates and V. A. Kostelecky, "Supersymmetric Matter Gravitino Multiplets," Nucl. Phys. B 248, 570 (1984).

[85] J. Maharana and J. H. Schwarz, "Noncompact Symmetries In String Theory," Nucl. Phys. B 390, 3 (1993) arXiv:hep-th/9207016.

[86] P. Fré, "Lectures on Special Kahler Geometry and Electric-Magnetic Duality Rotations," Nucl. Phys. Proc. Suppl. 45BC, 59 (1996) arXiv:hep-th/9512043]. 